\title{
WestVirginiaUniversity
}

THE RESEARCH REPOSITORY @ WVU

Graduate Theses, Dissertations, and Problem Reports

2014

\section{Functional characterization of Phosphodiesterase-4A in behavior and obesity}

Rolf T. Hansen III

West Virginia University

Follow this and additional works at: https://researchrepository.wvu.edu/etd

\section{Recommended Citation}

Hansen, Rolf T. III, "Functional characterization of Phosphodiesterase-4A in behavior and obesity" (2014). Graduate Theses, Dissertations, and Problem Reports. 583.

https://researchrepository.wvu.edu/etd/583

This Dissertation is protected by copyright and/or related rights. It has been brought to you by the The Research Repository @ WVU with permission from the rights-holder(s). You are free to use this Dissertation in any way that is permitted by the copyright and related rights legislation that applies to your use. For other uses you must obtain permission from the rights-holder(s) directly, unless additional rights are indicated by a Creative Commons license in the record and/ or on the work itself. This Dissertation has been accepted for inclusion in WVU Graduate Theses, Dissertations, and Problem Reports collection by an authorized administrator of The Research Repository @ WVU.

For more information, please contact researchrepository@mail.wvu.edu. 


\section{Functional characterization of Phosphodiesterase-4A in behavior and obesity}

\section{Rolf T. Hansen III}

A dissertation submitted to the West Virginia University Health Science Center Neuroscience Program in partial fulfillment of the requirements for the degree of:

Doctor of Philosophy in Neuroscience

\section{Committee:}

Han-Ting Zhang, Ph.D., Chair James O'Donnell, Ph.D. Adrienne Salm, Ph.D. Rae R. Matsumoto, Ph.D. Bernard Schreurs, Ph.D. Stanley Hileman, Ph.D.

WVU Neuroscience Program

Morgantown, West Virginia

\section{4}

Keywords: Phosphodiesterase, knock out, CAMP, CREB, cognition, behavior, PDE4A, anxiety, obesity

Copyright 2014 Rolf Hansen 


\section{$\underline{\text { Abstract }}$ \\ Functional characterization of Phosphodiesterase-4A in behavior}

\section{Rolf T. Hansen III}

Cognitive disorders such as depression, anxiety, and senescent-induced memory loss are a growing pandemic in the world. Anxiety is the most common psychiatric disorder in America, affecting upwards of 50 million people a year; depression has a lifetime prevalence of $20 \%$ of the population, and it's predicted pathological memory disorders such as Alzheimer's disease (AD) will reach exponential levels by the year 2050. While there are treatments for anxiety and depression, far less success has been reached with therapeutics for AD. Phosphodiesterases (PDEs) may be a potential novel target for future therapeutics aimed at combatting these psychiatric illnesses and neurodegenerative diseases. PDEs are a "super family" of 11 smaller enzyme families expressed throughout the body that degrade the cyclic nucleotides CAMP and/or cGMP, essentially acting as an off switch for intracellular cAMP and/or cGMP signaling. In particular, one member of this family termed PDE4 is highly expressed in regions of the brain that may play a role in these psychiatric disorders such as the prefrontal cortex (PFC), hippocampus, amygdala, and striatum. PDE4 has four subtypes ( $A, B, C$, and D), of which only PDE4C is not highly expressed in the brain. It has been found through the use of genetic manipulation that PDE4D is highly involved with memory and depressive-like behavior, and PDE4B is involved with anxiety and release of inflammatory factors from immune cells; however, the functional role that PDE4A plays in behavior was not known. The purpose of this dissertation was to characterize the functional role of PDE4A in behavior through the use of PDE4A KO mice, and a battery of behavioral and biochemical tests. We found that PDE4A KO mice displayed a strong anxiogenic profile, and a prolonged release of corticosterone in response to a stressor. In addition, they also displayed enhanced emotional memory. PDE4A $K O$ had no effect on depressive-like behavior, and decreased the training latency in the Morris Water Maze. PDE4A KO mice were also obese which was not due to hyperphagia or decreased locomotor activity. It was found that PDE4A KO decreased the levels of the major metabolic hormone T3. These results suggest that with further characterization, PDE4A may be a novel target in the future for anxiety or emotional memory disorders, as well as obesity. 


\section{Dedication}

This dissertation is dedicated to the best parents a son could ask for, Rolf and Jackie Hansen. Without your immeasurable love and support this accomplishment would not have been possible. Thank you for always loving me, and consistently being my solid foundation through both the good times and the bad.

This dissertation is also in memory of my late grandmothers. First, Peg Barth ("Nanny"), whose battle with Alzheimer's disease provided the catalyst for my interest in neuroscience, and second, Inez Hansen ("MeMa"), whose love and encouragement for hard work and higher education motivated me to pursue my dreams and to not settle for anything less than my goals. 


\section{Acknowledgements}

I would like to thank my mentor and advisor Dr. Han-Ting Zhang. His guidance, patience, and strong belief in me through the years have helped shape me into a well prepared, independent scientist.

I would also like to thank my committee members, Dr. James O’Donnell, Dr. Adrienne Salm, Dr. Rae Matsumoto, Dr. Bernard Schreurs, and Dr. Stan Hileman for their support, patience, understanding, and insight.

Additional thanks goes to Dr. Marco Conti at the University of California San Francisco for providing the PDE4A KO mice used in this study and various other collaborators such as Drs. Rae Matsumoto, James O’Donnell, James O’Callaghan, and Max Sokolov who provided instrumentation and equipment that helped this research be performed.

Lastly, I would like to thank current and past members of the Zhang and O'Donnell labs for their support. 


\section{$\underline{\text { Table of Contents }}$}

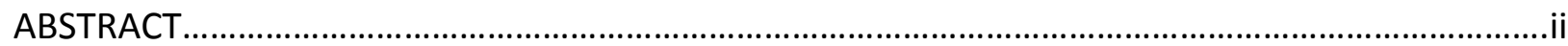

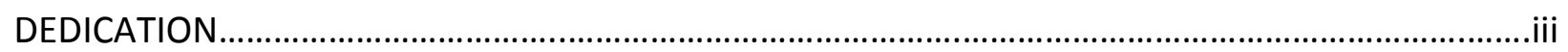

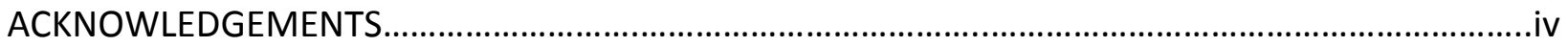

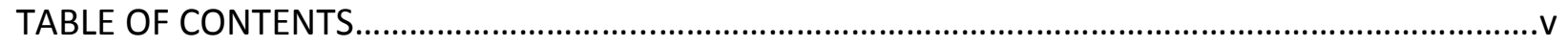

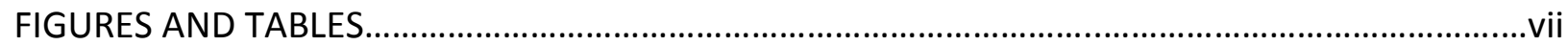

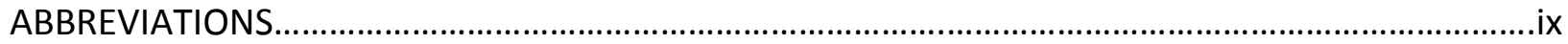

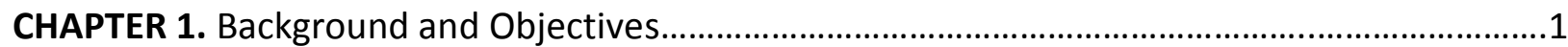

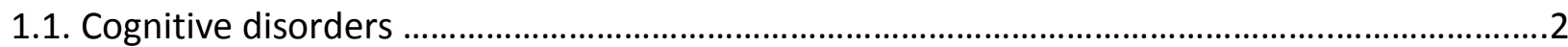

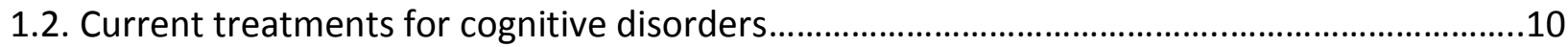

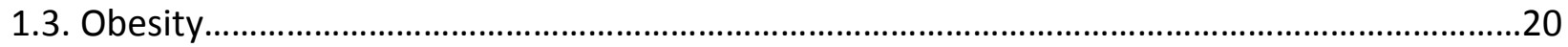

1.4. Need for new therapeutics in cognitive disorders and obesity ............................................23

1.5. Phosphodiesterase 4 and downstream signaling as a potential therapeutic for cognitive

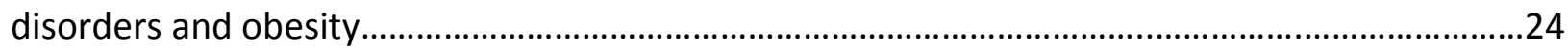

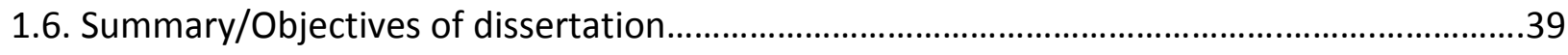

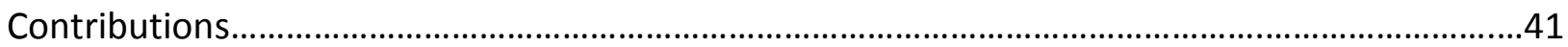

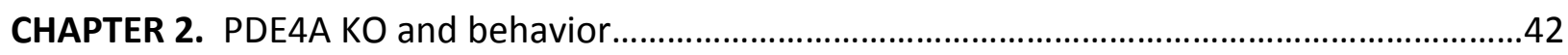

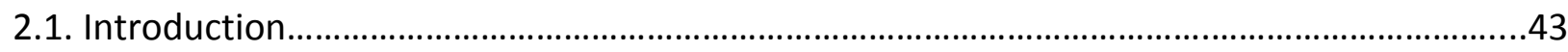

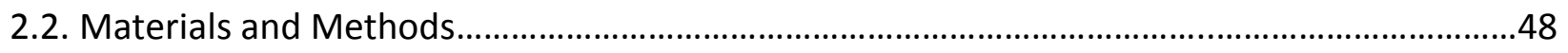

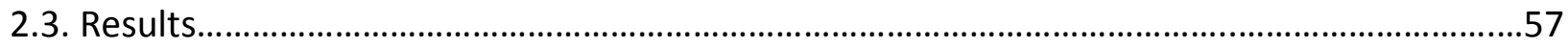

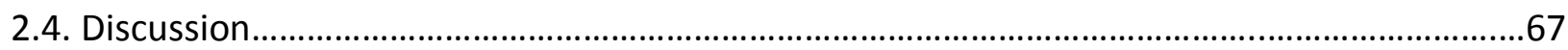

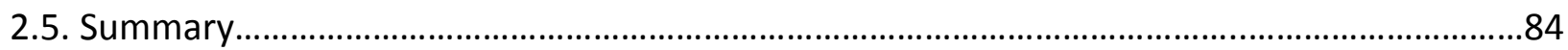

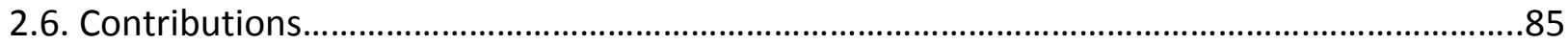


CHAPTER 3. PDE4A KO and obesity.

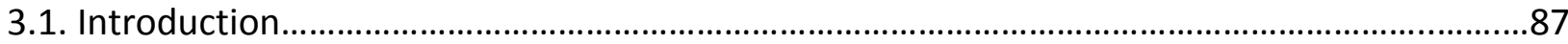

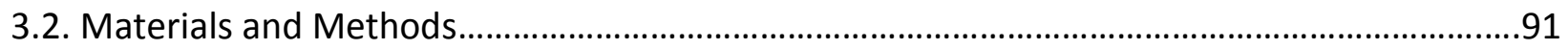

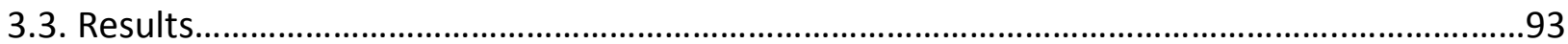

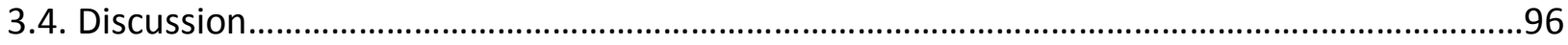

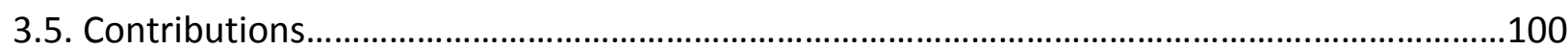

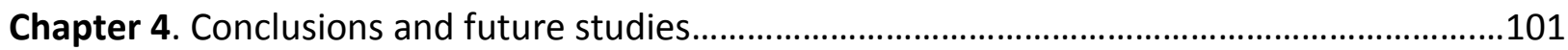

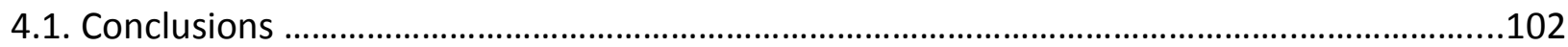

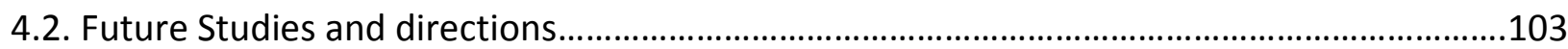

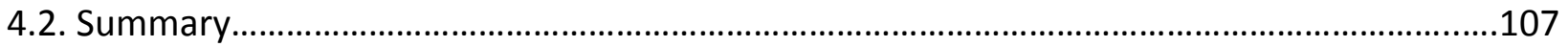

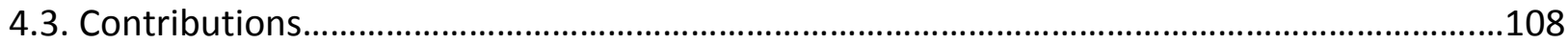

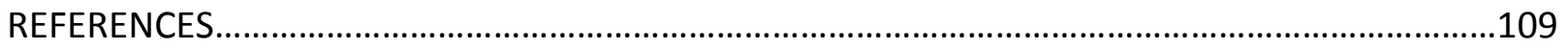

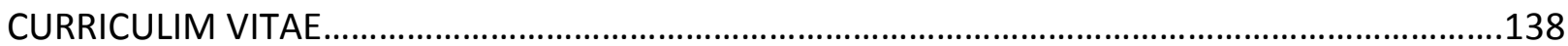




\section{Figures and Tables}

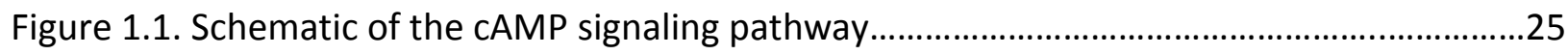

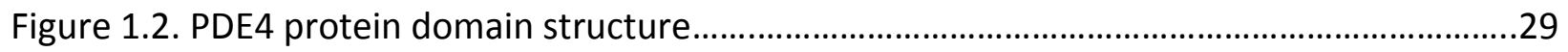

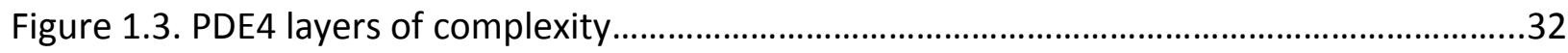

Figure 2.1. Behavioral paradigm for PDE4A KO studies..........................................................49

Figure 2.2. Effects of PDE4A KO on locomotor activity and rearing...........................................57

Figure 2.3. Effects of PDE4A KO on anxiety-like behavior in the elevated plus maze test............58

Figure 2.4. Effects of PDE4A KO on anxiety-like behavior in the holeboard test..........................58

Figure 2.5.Effects of PDE4A KO on anxiety-like behavior in the light dark transition test............59

Figure 2.6. Effects of PDE4A KO on anxiety-like behavior in the novelty suppressed feeding

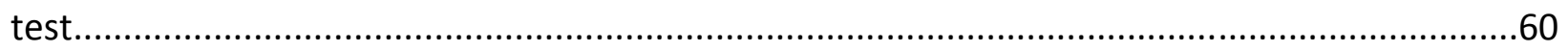

Figure 2.7. Effects of PDE4A KO on urine corticosterone levels ................................................60

Figure 2.8. Effects of PDE4A KO on behavior in the forced swim and tail suspension

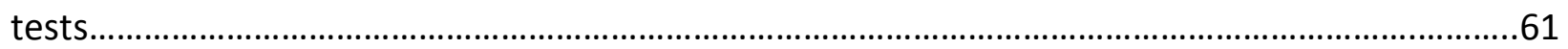

Figure 2.9. Effects of PDE4A KO on episodic-like memory in the object recognition test.............62

Figure 2.10. Effects of PDE4A KO on spatial memory in the Morris water maze test...................63

Figure 2.11. Effects of PDE4A KO on visual function in ERGs..................................................64

Figure 2.12. Effects of PDE4A KO on "emotional" memory in the step through passive avoidance

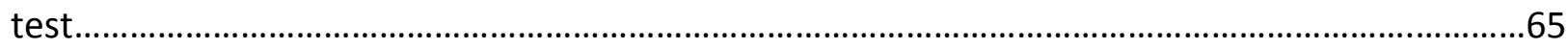

Figure 2.13. Effects of PDE4A KO on nociception in the hot-plate test.....................................66

Figure 2.14. Effects of PDE4A KO on emetic-like behavior......................................................66

Figure 2.15. Confirmation of null PDE4A expression in PDE4A KO mice.....................................67

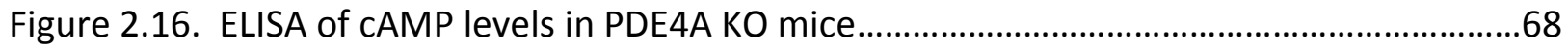

Figure 2.17. Protein levels of CREB/pCREB in PDE4A KO mice.................................................69 


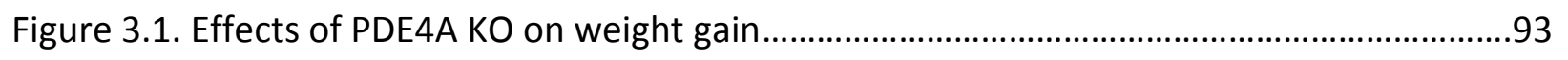

Figure 3.2. Effects of PDE4A KO on food consumption..........................................................94

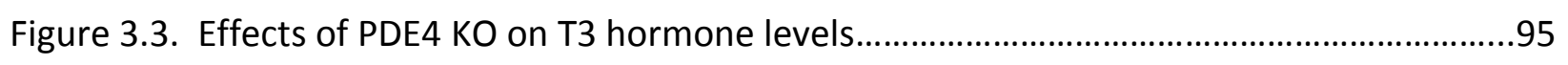




\section{List of Abbreviations}

Abbreviation

AAP

AC

$A D$

ADAS-cog

AgRP

AKAP

BDNF

BZD

CAMP

CBP

CGI

cGMP

CRE

CREB

CRF

DISC

DSM-V

ECT

EPAC

GAD

GR

HAM-A

HAM-D

\section{Meaning}

Atypical antipsychotic

Adenylyl cyclase

Alzheimer's disease

Alzheimer's disease Assessment Scale-Cognitive

Agouti-related peptide

A-Kinase anchoring protein

Brain derived neurotrophic factor

Benzodiazepine

3'-5'-cyclic adenosine monophosphate

CREB-binding protein

Clinical global impression scale

3'-5'-cyclic guanosine monophosphate

cAMP response element

CAMP response element binding protein

corticotropin-releasing factor (CRF)

Disrupted in schizophrenia

Diagnostic and Statistical Manual of Mental Disorders

Electroconvulsive therapy

Exchange protein directly activated by cAMP

Generalized-anxiety disorder

Glucocorticoid receptor

Hamilton rating scale for anxiety

Hamilton rating scale for depression 


\begin{tabular}{|c|c|}
\hline ICER & Inducible cAMP early repressor \\
\hline LTM & Long-term memory \\
\hline LTP & Long-term potentiation \\
\hline MAOI & Monoamine oxidase inhibitors \\
\hline MAPK & Mitogen-activated protein kinase \\
\hline MMSE & Mini-mental state examination \\
\hline MR & Mineralcorticoid receptor \\
\hline NPY & Neuropeptide Y \\
\hline OCD & Obsessive-compulsive disorder \\
\hline pCREB & phosphorylated CREB \\
\hline PDE & Phosphodiesterase \\
\hline PFC & Prefrontal cortex \\
\hline PKA & Protein kinase $A$ \\
\hline PTSD & Posttraumatic-stress disorder \\
\hline qEEG & Quantitative electroencephalography \\
\hline STM & Short term memory \\
\hline SNP & Single nucleotide polymorphism \\
\hline SNRI & Serotonin-norepinephrine reuptake inhibitor \\
\hline SSRI & Selective serotonin reuptake inhibitor \\
\hline T3 & Triiodothyronine hormone \\
\hline TAP & Typical antipsychotic \\
\hline TAPAS-1 & $\begin{array}{l}\text { tryptophan anchoring phosphatidic acid selective-binding } \\
\text { domain } 1\end{array}$ \\
\hline TCA & Tricyclic antidepressants \\
\hline TMS & Transcranial magnetic stimulation \\
\hline TRD & Treatment-resistant depression \\
\hline
\end{tabular}


TrkB

VNS tyrosine kinase b receptor(TrkB)

Vagus nerve stimulation 
Chapter 1

\section{BACKGROUND AND OBJECTIVES}




\subsection{Cognitive disorders}

Cognitive disorders such as pathological anxiety, depression, and both non-pathological and pathological memory loss are a growing pandemic in the United States and the rest of the world. The following sections will review what defines these disorders, current treatments, and the limitations and side effects of current treatments.

\subsubsection{Pathological Anxiety. Anxiety is a physiological, psychological, and behavioral state induced in} animals and humans by a threat to well-being and survival, either actual or potential (Steimer 2011). The "potential" aspect of anxiety is what directly contrasts it from "fear"; fear is a reaction of the body to immediate and present danger, while anxiety is a reaction of the body to future events that may or may not occur. Due to the fact that anxiety is anticipatory, it can be argued that anxiety is an essential behavior necessary for the survival of species, and probably evolved as an adaptive survival mechanism for animals to avoid danger, thus increasing the chances of mating and passing on their genes. While these behaviors may have evolved to be beneficial, in pathological anxiety animals or humans are unable to appropriately adjust to varying degrees of stress whether real or perceived. Particularly in humans, pathological anxiety has manifested as the most common psychiatric disorder in the country, affecting around $18 \%$ of Americans and costing the American economy over 50 billion dollars a year (Greenberg et al. 1999; Kessler et al. 2010). The diagnostic and statistical manual of mental disorders $\mathrm{V}$ (DSMV) is the standard diagnostic manual for mental health physicians across the country to diagnose psychiatric disorders, and has classified anxiety into 12 separate disorders including: separation anxiety disorder, selective mutism, specific phobias, social 
anxiety disorder, panic disorder, panic attack (specifier), agoraphobia, generalized anxiety disorder, substance/medication-induced anxiety disorder, anxiety disorder due to another medical condition, other specified anxiety disorder, and unspecified anxiety disorder. This current list of anxiety disorders excludes two additional anxiety related disorders that were previously included in the DSM-IV-TR known as obsessive-compulsive disorder (OCD) and posttraumatic stress disorder (PTSD); these two disorders have now been moved to different sections in the DSM-V. From this list of 12 anxiety disorders, the most putatively common and well known condition is generalized anxiety disorder (GAD). Generalized anxiety disorder is a condition that develops slowly, involving both environment, life stresses, and genetics, with an average onset around 31 years of age. In this condition, individuals are unable to appropriately adjust to the realistic stressors of life. They are filled with anxiety, and have difficulty extinguishing their concerns about situations in life, even though these situations might never occur, or their anxiety is significantly more intense than the situation warrants (Baldwin et al. 2013). Normally because of this anxiety they have trouble sleeping, and can have significant problems functioning socially or maintaining a job (Katzman 2009). Due to this constant pathological anxiety and subsequent stress that is placed on their bodies, physical symptoms such as hot flashes, nausea, loss of breath, headaches, muscular tension and instability, and the frequent feeling of needing to go to the bathroom begin to manifest which can result in a positive feedback loop, making the internal anxiety even worse. For the remainder of this dissertation unless otherwise specified, GAD will be the main focus of anxiety-related discussion. 
Typically in the clinic, the diagnosis of GAD requires that the individual have persistent anxiety and worry on most days of the week for a period of 6 months or longer. In addition, at least 3 additional criteria must be met such as restlessness, concentration problems, sleep disruption, muscular tension, irritability, or fatigue. To provide an objective measure of an individual's anxiety, the Hamilton rating scale for anxiety (HAM-A) or the clinical global impression scale (CGI) are often used (Sramek et al. 2002). With the HAM-A, the individual is scored on 14 different items that can be ranked on a scale of 0-4. The scores are then combined with a maximum score of 56 possible. The score of the HAM-A correlates with the severity of anxiety, with 17 or less indicating mild anxiety, 18-24 intermediate anxiety, and 25 and up severe anxiety (Hamilton 1959). The CGI scale is an additional scale that is often used by mental health professionals for a broad spectrum of disorders. The CGI scale is a 3 component scale that rates the severity of a person's disorder (anxiety in this case), their global improvement over time, as well as the efficacy of a treatment. The severity of the person's disorder is ranked on a 7 point scale rated from 1 (normal) to 7 (very severe); the overall improvement of the patient is ranked 1 (very much improved) to 7 (very much worse), and the overall effectiveness of a drug is ranked as unchanged or worse, minimal, moderate, or marked. For patients that aren't assessed for certain values a " 0 " is assigned (Guy 1976). Perhaps the double-edged sword component of the CGI is that it is subjective. This scale requires a clinician with experience in the treatment of anxiety disorders to be able to compare to what is "normal" and what they think the severity of the patient is. Due to this subjectivity, this scale is more than just a "checklist" and perhaps brings a human element into the diagnosis of the 
disorder, however like many things this rating could differ between various clinicians (Busner and Targum 2007).

1.1.2. Depression. Depression is a chronic psychological condition characterized by extreme sadness for prolonged periods of time, and loss of motivation and reward also known as anhedonia. Like anxiety, depression is a very prominent psychiatric disorder which affects about $17 \%$ of Americans, is one of the leading causes of disability worldwide, and is the $8^{\text {th }}$ leading cause of death in the United States. The high prevalence of depression has a huge economic burden on America, which is estimated to be around \$30-44 billion dollars a year (Greenberg et al. 1999). While the evolutionary advantage of depression is difficult to determine, like anxiety, many believe that depression may have evolved to cause "psychic pain", which in turn causes the individual to try to avoid future experiences for events that caused that pain which could possibly be a life threatening experience. However, like anxiety, this system can become extremely dysregulated in humans, and the pathological depression which we know today is a direct result of that dysregulation.

Some diagnostic criteria for depression include: significant changes in weight, changes in the sleep cycle, increased agitation or catatonic states, significant fatigue or loss of energy, recurrent suicidal thoughts, excessive feelings of worthlessness or guilt, and finally a diminished ability to concentrate and think in parallel with indecisiveness. The severity of depression can be scored with the CGI, and also the Hamilton rating scale for depression (HAM-D) which is the depression counterpart to the HAM-A (Hamilton 1967). An additional test to measure depression is known as the Beck Depression Inventory (BDI). This test requires the evaluation of 21 different items that can be ranked 0 to 3 . The total score is looked at and a score larger 
than 10 indicates mild to moderate depression while a score over 18 indicates moderate to severe depression (Forkmann et al. 2011).

1.1.3. Memory disorders. There are a plethora of cognitive conditions and external causes which can manifest as memory loss, however, normal senescent-induced memory loss and pathological aging (Alzheimer's disease, AD) are two of the most putatively well known. Aging, also known as senescence, can be defined according to the Dorland Medical Dictionary as "the process of growing old, especially the condition resulting from the transitions and accumulations of the deleterious aging processes." The knowledge of the deleterious effects of senescence is not a recent or novel discovery; in fact, it has been known for thousands of years that certain biological processes become less efficient with increasing age while other processes remain intact (Berchtold and Cotman 1998; Roman 1999).

In particular, memory decline has been one of the most noted and observed changes correlated with increased biological age. However, different types of memory are affected to varying degrees, and not all aged individuals are affected (Rowe et al. 1998; Perls 2006).

Among the forms of memory most commonly affected in aged individuals are working memory, episodic memory, spatial memory, and long term memory (LTM). The other forms of memory more commonly spared from the aging process include short term memory (STM), procedural memory, and semantic memory (Light 1992). While STM and working memory are similar in that they both involve memory over a limited period of time, working memory differs from STM in that it requires the active manipulation of information being held in attention, like baking a cake or doing arithmetic. In contrast, STM is the simple maintenance of that information over a short period of time, such as remembering a phone number. 
It is also important to identify the main differences between non-pathological aging and pathological aging such as AD. Previous dogma suggested that alongside normal senescence there was a significant decrease in the neuronal number (Brody 1955; Shefer 1973; Henderson et al. 1980). Indeed, initial studies showed that aged brains had significantly less neurons, and thus the conclusion was made that with aging neuronal death occurred. However, recent studies have mostly proven this to be untrue (Peters et al. 1998). It is now generally believed that with senescence there is no significant decline in the number of neurons, and previous findings showing neuronal loss with aging have been discovered to be an artifact due to the processing of aged brains (Haug 1985). Young brains actually shrink much more in the fixation process, making them much denser (neurons per unit of area), and at first glance appearing to grant them higher neuronal counts over aged brains (Peters et al. 1998). When this confounding variable is accounted for and through the use of unbiased stereological methods, it has been shown that the aged brain has no significant neuronal loss (Giannaris and Rosene 2012). While the aged brain is slightly smaller in weight and volume (Skullerud 1985), this is most likely caused by a decrease in neuronal size and somatic density (Terry et al. 1987). An additional theory was that alongside senescence there was a loss of synapses in the brain (Geinisman 1999). However some studies have shown that there is actually no change of synapses in hippocampal CA1 of aged rats, suggesting that memory deficits observed in senescence may in fact be functional in nature (Geinisman et al. 2004). This is further confirmed through findings that microcolumns in area 46 of the aged rhesus monkey lose strength with age despite the fact the neuronal density and number is unchanged (Cruz et al. 2004). 
$A D$ is a significant diversion of the aging process, and has many differences from normal aging. After being diagnosed with AD, there is an average survival of 5-10 years, and individuals over the age of 85 have a 50\% chance of contracting this disease (Drachman 2006; Herrup 2010; Podtelezhnikov et al. 2011). While normal aging has now been shown to have no significant changes in the neuronal number, $A D$ is defined by massive atrophy in the brain which results in cognitive deficits much more severe than gradual senescent-related decline (Scheltens et al. 1995; Jack et al. 1997; Drachman 2006; Glisky 2007; Herrup 2010;

Podtelezhnikov et al. 2011). Why and how normal aging turns into AD is currently unknown, but it is known that other than familial-inherited $A D$, age is the largest risk factor for sporadic $A D$; in fact, some have suggested that $A D$ is an aberrant form of aging that rapidly accelerates the aging process of the brain (Podtelezhnikov et al. 2011). In a fascinating study looking at gene expression in aged and demented brains, it was found that AD brains had significant changes in genes involved with lipid metabolism, the stress response, inflammation, and peculiarly those involved with cell adhesion, migration, and morphogenesis, baring a striking resemblance to patterns of gene expression also observed in epithelial mesenchymal transition (EMT) tissues (Podtelezhnikov et al. 2011). These findings raise fascinating points and reveal the similarity AD has in common with EMT, suggesting a major transformation in brain tissue physiology and receptor signaling is occurring in $A D$. When the data obtained from the normal aged patients was extrapolated, it was predicted that in non-pathological aging, normal subjects would not reach AD cognitive-like levels until 140 years of age (Podtelezhnikov et al. 2011). 
Thus, in normal aging, even though mild memory loss is occurring and there is a gradual decrease in brain weight, it seems as if there is no significant neuronal death occurring and that most of the observed behavioral deficits may be functional in nature. This is in direct contrast with $A D$, which may start out as an aberrant aging process which is functional in nature, but then quickly progresses to anatomical changes through large scale inflammation, gliosis, and loss of neurons. Due to the fundamental differences in these two aging processes, it is more than likely that no one "silver-bullet" therapy exists for both conditions in all severities. The more feasible approach may be that multiple therapies would need to be used for the different processes, and different times in the progression and severity of AD.

The diagnosis of $A D$ is often a difficult one, and requires health care professional to exclude many additional factors which could be the cause of the declining mental health such as: delirium caused by urinary tract infections, vascular dementia, Parkinson's induced dementia (Lewy-body disease), frontotemporal dementia, hydrocephalus, Huntington's disease, traumatic brain injury, vitamin deficiencies, endocrine conditions, prion diseases, liver disorders, brain tumors, and immune disorders just to name a few. The level of cognitive impairment is often measured through the administration of the mini-mental state examination (MMSE). This is a 30 point questionnaire which asks a number of simple questions testing several categories such as: orientation to time, orientation to place, registration, attention and calculation (tests working memory as well), recall, language, repetition, and complex commands. The various scores from these questions are summarized, and the total score out of 30 is used to make an assessment on the level of cognitive impairment. Typically a score of 27 or higher indicates average cognition; scores less than 9 indicate sever cognitive impairment, 
scores 10-18 indicate moderate impairment, and scores 19-24 indicate mild cognitive impairment (Mungas 1991). Interestingly, it has also been shown that the MMSE can be used to differentiate various dementias from one another (Jefferson et al. 2002; Ala et al. 2002; Palmqvist et al. 2009). In addition to the MMSE, the Alzheimer's disease Assessment ScaleCognitive (ADAS-cog) is a separate scale that can be used to monitor the severity and progress of $A D$ (Graham et al.)

\subsection{Current treatments for cognitive disorders and drawbacks}

1.2.1 Treatments for anxiety. There are multiple modes of therapy that can be prescribed to help combat pathological anxiety in humans such as cognitive and behavioral therapy, medications, and alternative eastern therapies.

Cognitive and behavioral therapies are a form of counseling that teaches anxiety patients techniques for gaining control over their thoughts and concerns. In particular, they are trained to distinguish actual concerns from potential or even fictitious thoughts that are unrealistic. This is done through the analysis of their thoughts, behaviors, and feelings and the recognition of patterns within themselves that lead to destructive or disadvantageous outcomes. Once they are able to recognize the destructive thoughts and behaviors, the patients are then able to more easily modify their thoughts and behaviors to cope with their condition (Deacon and Abramowitz 2004). For anxieties which refer to specific phobias, typically exposure therapy is the treatment of choice. With this psychotherapy, anxiety patients are slowly exposed to concepts and pieces of their phobia, in hopes of gradually habituating them to it (Gunter and Whittal 2010). For instance, if a person was afraid of flying in planes, exposure therapy might first start with showing the people pictures of a plane, or 
persons inside a plane smiling and laughing. Next, they could have the person pack a bag as if they were getting ready for an actual trip. Additional steps could be driving to the airport, and then sitting at the gate. Eventually, through gradual habituation and training the person is eventually able to confront their phobia of flying.

In addition to behavioral- and psycho-therapies, medications such as benzodiazepines (BZDs), selective serotonin reuptake inhibitors (SSRIs), azapirones, beta blockers, antipsychotics, and pregabalins are also a common choice for many health care professionals in the treatment of anxiety.

BZDs, which are the most commonly prescribed medication for the treatment of acute anxiety (Stahl and Ph 2002), are GABAergic allosteric modulators first discovered in the 1960s that bind to the GABA receptor, and potentiate the action of GABA. This GABAergic potentiation then causes increased flow of $\mathrm{Cl}^{-}$through the GABA receptor, and subsequent hyperpolarization and inhibition of the cell (Argyropoulos et al. 2001). Some of the most commonly prescribed BDZs are Alrpazolam (Xanax), Lorazepam (Ativan), Clonazepam (Klonopin), and Diazepam (Valium) (Stahl and Ph 2002). BZDs are most commonly prescribed for short term anxiety, and tend to work rather well (Davidson 2009); however, the benefits of BZDs are often outweighed by their strong negative side effects. While BZDs work well at short term relief of anxiety, they are not suited for chronic administration. In particular, BZDs are plagued by their strong side effects, which can include sedation and psychomotor impairment. In addition, BZDs can also be addictive, which can lead to withdrawal symptoms and rebound symptoms further complicating the mental health of the individual (Argyropoulos et al. 2001; Sramek et al. 2002; Stahl and Ph 2002; Katzman 2009). Interestingly, BZDs have also developed 
a strong negative stigma which prevents or makes most patients uncomfortable with their use which can lead to decreased patience compliance (Sramek et al. 2002).

While BZDs are most adept at treating immediate relief of anxiety, the general opinion of clinicians suggests that the ultimate goal of treatment is long term relief of anxiety-like symptoms. Selective serotonin reuptake inhibitors (SSRIs) and serotonin-norepinephrine reuptake inhibitors (SNRIs) have become the recommended first-line therapies for the long term maintenance and treatment of GAD. SSRIs inhibit the serotonin transporter which is responsible for the reuptake of serotonin from the synaptic cleft back into the neuronal soma for reuse and degradation. Thus, SSRIs potentiate the effects of serotonin by prolonging its persistence in the synaptic cleft. SNRIs are similar, however, they are able to inhibit the uptake of both serotonin and norepinephrine. Some of the most commonly prescribes SSRIs and SNRIS for anxiety are as follows: paroxetine (Paxil $\left.{ }^{\circledR}\right) 12 \%$ of anxiety cases, venlafaxine $\left(\right.$ Effexor $\left.^{\circledR}\right) 6 \%$, sertraline $\left(\right.$ Zoloft $\left.^{\circledR}\right) 5 \%$, citalopram (Celexa ${ }^{\circledR}$, or the newer escitalopram known as Lexapro ${ }^{\circledR}$ ) $4 \%$, and Nefazodone (Serzone ${ }^{\circledR}$ ) 4\% (Stahl and Ph 2002).

While SSRIs and SNRIs are better at the long-term treatment of anxiety over BZDs, they are not without a plethora of side effects and drawbacks of their own. Perhaps one of the initial major drawbacks is their therapeutic lag, with an onset of about 1-2 weeks. In addition, there are many common side effects of these drugs such as nausea, vomiting, headaches, insomnia, diarrhea, weight change, paradoxical behavior (i.e., increased depression or anxiety) suicidal thoughts, and dizziness just to name a few. These drugs also need to be tapered off, and even with this gradual weaning of the drug withdrawal can still manifest and persist for 1-2 weeks (Argyropoulos et al. 2001). 
A third class of molecules which can be used to treat GAD are known as azapirones. Azapirones have a different mechanism of action than BZDs and SSRIs, and work by acting as a partial agonist for the 5-HT1A receptor (Chessick et al. 2006). The serotonin 5-HT1A receptor is Gai bound which leads to inhibition of adenylyl cyclase and subsequently decreased levels of CAMP (Olivier et al. 1999). Buspirone $\left(\right.$ Buspar $^{\circledR}$ ) is the most commonly used azapirone, and is prescribed in about $9 \%$ of the drug treatments for GAD (Stahl and Ph 2002). While buspirone has its advantages over BZDs in that it is not associated with withdrawal, addiction, or tolerance, it has its own list of drawbacks and side effects. Buspirone for one has a long therapeutic lag of 2-3 weeks, thus it is not optimal for the treatment of acute anxiety (Katzman 2009). It has also been reported by anxiety patients that buspirone is not as effective as BZDs and SSRIS/SNRIs and thus patient compliance is lower with buspirone treatment (Chessick et al. 2006). In addition, buspirone has a short half-life which can require patients to take it more often throughout the day which can decrease patient compliance (Sramek et al. 2002).

Some less commonly used drugs for GAD include beta adrenergic blockers and antipsychotics. Beta blockers are more often used for panic disorders, specific phobias, and social anxiety - mainly because they are able to control the peripheral somatic symptoms that are the defining characteristics of many of these disorders; however, in certain cases they are sometimes prescribed for the treatment of GAD (Argyropoulos et al. 2001). Antipsychotics are also sometimes used for the treatment of nonpsychotic anxiety, however their use is controversial and still somewhat inconsistent and reserved as a last resort option for individuals that have experienced treatment-resistant GAD. Antipsychotics can be broken down into "typical" antipsychotics (TAPs) which are the first generation drugs developed in the 1950s that 
are dopamine receptor antagonists, and "atypical" antipsychotics (AAPs) developed in the 1990s which tend to have less motor side effects than their predecessors. Trifluoperazine, which is a first-generation TAP, has FDA approval for use in GAD. In addition the TAP perphenazine has also been used in combination with amitriptyline, a tricyclic antidepressant (Pies 2009), for the treatment of GAD. While the TAPs have FDA approved labeling for the treatment of GAD, that does not exclude the use of AAPs by clinicians for the use of GAD as an off label use (Summary 2011). There are some studies showing that these AAPs may have an effect on anxiety (Pies 2009), whereas other studies have yielded inconsistent results on the legitimacy of using AAPs as a therapy for treatment-resistant GAD (Lorenz et al. 2010). Thus more studies are needed on the use of AAPs in anxiety before FDA approval is obtained.

There are other drugs that are also approved for anxiety in other parts of the world that are not approved by the FDA in America. One such drug is known as Pregabalin. Pregabalin is a high-affinity ligand for the alpha-2-delta subunit of the $P / Q$ calcium channel. While it has been shown that this ligand can be mildly effective in the treatment of GAD, it is currently not approved in the United States (Katzman 2009; Baldwin et al. 2013). An additional drug approved in Europe and Australia is Agomelatine. Agomelatine is a melatonin receptor 1 agonist that has been reported to decrease the severity of anxiety-like symptoms as measured by the HAM-A in several clinical trials (Stein et al. 2008; Stein et al. 2013; Stein et al. 2014). While Agomelatine was tested in the United States, it did not make it through clinical trials due to weak efficacy and possible liver damage (http://clinicaltrials.gov/ct2/show/results/NCT00411099). 
Lastly, there are also alternative forms of medicine available for anxiety such as acupuncture, yoga, and promoting a healthier lifestyle through exercise and proper nutrition. It was found in a placebo-controlled, randomized, double-blind study that after 10 sessions of verum acupuncture, patients showed a significant improvement in anxiety as measured through the CGI (Eich et al. 2000). While the mechanisms for how acupuncture works are unknown, it has been suggested that acupuncture may work through activation of the parasympathetic nervous system via activation of the vagus nerve. Supporting this theory are studies which examined acupuncture in the large intestine 4 regions (LI4) vs a placebo acupuncture at a non-essential sham area. This study used both quantitative electroencephalography (qEEG) and heart rate variability (HRV) to examine how these treatments affected EEG brain patterns and the low frequency/high frequency ratio of the heart. They found LI4 acupuncture had a significant effect on the $\alpha 1$ waves of the brain, and also significantly increased the HF ratio of the heart and decreased heart rate a minute after simulation, indicating an activation of the vagus nerve (Streitberger et al. 2008). This agrees with with clinical studies showing that vagus nerve stimulation is an effective treatment for mood disorders (George et al. 2007).

Yoga is another possible alternative treatment for anxiety, however, the results on the efficacy of yoga in lowering clinical severity are inconsistent and need further clarification (Kirkwood et al. 2005). Exercise and healthy lifestyle choices are a broad treatment which generally improve most diseases in a non-specific manner. Indeed, it has been shown that exercise can help make people more resilient to stress and reduce anxiety-like symptoms (Broman-Fulks et al. 2004). However, exercise is not without its own downfalls as a treatment 
option, as this is a difficult life transition for many individuals to implement due to time or willpower, and may also be difficult for certain individuals with impairments that may prohibit them from active exercise (Salmon 2001; Ströhle 2009).

1.2.2. Treatments for depression. The treatments for depression are remarkably similar to those used for the treatments of GAD, and this should come as no surprise in that depression exhibits a 63\% comorbidity with GAD (Davidson 2009). The first-generation antidepressants were the monoamine oxidase inhibitors (MAOI) and tricyclic antidepressants (TCAs). These drugs were both discovered around the 1950s, and soon thereafter became strongly used in the treatment of depression. The first MAOI, iproniazid, was initially developed for tuberculosis when it was noticed that patients taking this experienced improvements in their mood and heightened euphoria. The MAOls work by inhibiting the monoamine oxidase enzyme, which is an enzyme responsible for the breakdown of serotonin and norepinephrine. The TCAs followed shortly thereafter with the discovery of imipramine (Tofranil) which was originally developed for schizophrenia (Lieberman 2003). The TCAs work by increasing levels of norepinephrine and serotonin in the synaptic cleft. While these antidepressants were hailed as "miracle drugs" when they were first discovered, their use has greatly diminished over the last decades due to safety concerns.

The SSRIs and SNRIs which are the newer "second -generation" line of antidepressants began to make an appearance in the late 1980s, and began replacing the TCAs and MAOIs in terms of first-line therapy choice (Olfson and Marcus 2009). SSRIs are the most commonly prescribed medication for depression and have been estimated to account for nearly $70 \%$ of all antidepressant drugs (Olfson and Marcus 2009). The most commonly prescribed SSRIs are 
paroxetine (Paxil), sertraline (Zoloft), citalopram (Celexa), and fluoxetine (Prozac). Although all of these SSRIs work by inhibiting serotonin uptake, they have differences in their pharmacokinetic profile which may offer unique pros and cons for each drug over one another (Magni et al. 2013). As mentioned previously, while SSRIs and SNRIs are much safer than the first-generation MOIs/TCAs, they have a long therapeutic lag and a plethora of side effects.

It is very common for patients not to respond to monotherapy for depression, in fact typically only $30 \%$ of people respond to one therapy. Thus depression typically requires much experimentation and combination-adjunctive therapy (Khan et al. 2012). It has been found that combining both cognitive therapy and or multiple drug therapies typically delivers the highest success rate (Shelton et al. 2010). This lack of success with single antidepressant efficacy is known as "treatment-resistant depression" (TRD). While the pharmacotherapies and nonpharmacological therapies such as cognitive therapy are typically first-line treatments, there are several options available to clinicians for those who are resistant. Electroconvulsive therapy (ECT) is one of the oldest therapies for depression, and is still used today albeit as a late choice therapeutic for TRD. While ECT is highly effective and works in about $50-60 \%$ of treatment-resistant cases, it has a highly negative stigma in our society. In addition, the main side effects of ECT are cognitive impairment with loss of memory as the most common deficit observed (Lisanby 2007; Shelton et al. 2010). Vagus nerve stimulation (VNS) is an additional option for TRD. Originally used for the treatment of epilepsy, it was approved for the treatment of depression in 2005 by the FDA. Initial studies have shown promising results for VNS, but since this is an invasive procedure it is only used for severe TRD. Side effects of VNS can include neck pain, difficult breathing, hoarseness, changes in voice, and coughing (George 
et al. 2007; Shelton et al. 2010). Transcranial magnetic stimulation (TMS) is a newer technique approved by the FDA in 2008 that has gained traction as a possible treatment for depression. TMS essentially is able to stimulate and "turn on" cortical networks through application of a magnetic field delivered from a coil placed above regions in the skull. Initial studies suggest TMR can be highly effective in TRD with success rates as high as $65 \%$, however more evidence is needed as the results are still inconsistent (Shelton et al. 2010). TMS is also regarded as being a safe treatment with very few side effects. There has been one reported case of seizures after TMS (Conca et al. 2000) and also several observances of induced mania (Sakkas et al. 2003).

Similar to anxiety, there are also many alternative therapies that can be used for depression such as yoga, acupuncture, and exercise. As described previously, these therapies have been shown to work in certain individuals, but seem to be hampered by lack of consistent evidence-based efficacy and/or difficulty with patient compliance (Eich et al. 2000; Salmon 2001; Broman-Fulks et al. 2004; Kirkwood et al. 2005; Streitberger et al. 2008; Wang et al. 2008; Ströhle 2009).

1.2.3. Treatments for memory disorders. While progress has been made in the development of therapeutics for anxiety and depression, there has been a significant bottleneck in the development of AD-related therapeutics. Currently there are only 5 approved drugs for AD in two different classes: the anticholinesterases (donepezil, Aricept ${ }^{\circledR}$; galantamine, Reminyl ${ }^{\circledR}$; rivastigmine, Exelon ${ }^{\circledR}$; and tacrine, Cognex ${ }^{\circledR}$ ), and the NMDA receptor antagonists (memantine, Namenda ${ }^{\circledR}$ ) (Raina et al. 2008). 
The anticholinesterase class of drugs is based upon the cholinergic hypothesis of AD, and the observation that acetylcholine levels are lowered in the brain of AD patients (Richter et al. 1980). It is believed that if these acetylcholine levels are restored, then the cognitive deficits associated with the disease could possibly be attenuated (Lleó et al. 2006). The cholinergic class of drugs raises acetylcholine levels by inhibiting the enzyme acetylcholinesterase, which is the enzyme that is responsible for the catabolism of acetylcholine. These various cholinergic drugs while having the same target, have different pharmacokinetics which is the main distinction between one another (Lleó et al. 2006). The first cholinergic drug ever approved for AD was tacrine (Crismon 1994). Since then, tacrine has been slowly replaced by the newer cholinergics most likely due to increased safety concerns, as tacrine was shown to have possible hepatotoxicity issues. While these cholinergic drugs are commonly prescribed by clinicians, they normally do so because of lack of options. Studies have shown while the clinical results seen by these drugs are significant over placebo, they are clinically very mild and demonstrate only small improvements in the MMSE and ADAS-cog (Lleó et al. 2006; Raina et al. 2008).

The second class of drugs approved for use in AD is the NMDA receptor antagonist memantine. The therapeutic rationale behind NMDA antagonism has its roots in the belief that glutamatergic excitotoxicity may be occurring in AD (Molinuevo et al.; Danysz et al. 2000; Hynd et al. 2004). While glutamate is an essential neurotransmitter in the brain and instrumental in the mediation of memory, too much of it can be damaging to neurons. It is believed that in the $A D$ brain this excitotoxicity may be occurring, thus memantine is believed to be able to stop this excitotoxicity through antagonism of NMDA-glutamate receptors. While the theory behind this is sound, the clinical efficacy of memantine has fallen short; it has been shown that memantine 
has inconsistent and very mild, albeit significant effects on cognitive symptoms (Lleó et al.

2006; Raina et al. 2008). Even though memantine may show a slight improvement on cognitive symptoms, it has been shown to have little efficacy in halting the course of AD pathology.

\subsection{Obesity}

Obesity is an additional disorder that has risen to the ranks of a national pandemic, affecting $\sim 30 \%$ of the population, and costing the United States over 150 billion dollars a year (Ogden et al. 2012). Obesity is also major risk factor for leading causes of death such as cardiovascular disease, stroke, and diabetes. Obesity is defined as a body mass index of over $30 \mathrm{~kg} / \mathrm{m}^{2}$, or a body fat percentage over $25 \%$. Sadly, obesity is one of the most preventable disorders in the country; however, due to cultural changes such as poor dietary choices, sedentary living, and overabundance of affordable, easily obtainable trans-saturated fats, it has become a pandemic to which our country is currently losing. These statements are made evident by the fact that zero states have been able to reduce their obesity rate below $15 \%$ as set forth in the United States healthy people 2010 pledge (Services 2010).

While at first one may think that obesity is significantly removed from mental illness because it is a peripheral disorder, recent research has shown that anxiety, depression, and obesity are not mutually exclusive, but may in fact be very closely related to each other. Obesity has been strongly linked to anxiety and depression, and exhibits a high comorbidity (Simon et al. 2007); however, a causal relationship between the two is still unclear. Due to the complicated mechanisms of these disorders it is still unknown which one is the catalyst, or if they are mutually influential; this complex relationship needs further characterization 
1.3.2. Treatments for obesity. There are currently many different therapeutics that can be used to combat obesity. The most obvious of these therapies and the first choice of many doctors is through exercise and proper nutrition. Diet and physical activity are typically recommended for people that have milder forms of obesity with a BMI in a range of 25-30 (Fisher and Schauer 2002). However, exercise and proper nutrition are often very difficult for most individuals and have a very low success and compliance rate. In addition, there is a high remission rate in those that are successful due to the body trying to reset itself to its "programmed weight".

An additional approach to combat obesity is through pharmacological intervention. Pharmacological interventions are not normally used until individuals have reached a BMI of around 30 (Fisher and Schauer 2002). There are several therapeutic strategies an anti-obesity drug can follow such as appetite suppression (anorectic), interference with absorption of nutrients, or increased metabolism of nutrients (Klonoff and Greenway 2008). While there were 4 FDA-approved drugs for the labeled treatment of obesity: Orlistat, diethylpropion, phentermine-topiramate, and sibutramine (Campfield 1998; Moyers 2005; Eckel 2008; Ioannides-Demos et al. 2011), recently the FDA has pulled many of the drugs for safety concerns (Colman et al. 2012). Currently orlistat is the only FDA approved drug for obesity on the market (Colman et al. 2012). Orlistat is a pancreatic lipase inhibitor that works by preventing the absorption of fat in the small intestine. Orlistat has been shown to be mildly effective in reducing weight, and is relatively safe. Since orlistat prevents the absorption of fat, the major side effects are fecal incontinence, flatulation, and oily discharge from the bowels (Campfield 1998; Moyers 2005; Eckel 2008; loannides-Demos et al. 2011). 
While the FDA now only has 1 approved drug for obesity, that does not prevent clinicians from using other drugs to combat obesity in "off label" uses. One such example is the use of bupropion (Wellbutrin ${ }^{\circledR}$ ) as an off target therapy for obesity (Moyers 2005). In addition, there are also clinical trials underway for new obesity-fighting drugs such as Zonisamide. So far Zonisamide has shown promise (loannides-Demos et al. 2011; Nguyen et al. 2013; Gadde et al. 2014), and if approved may be combined with bupropion to form a synergistic novel drug that could combat obesity.

The large list of non FDA approved obesity drugs such as diethylpropion, phenterminetopiramate, sibutramine, rimonabant, Lorcaserin, and amfepramone are representative of the difficulty in developing a safe anti-obesity agent. It is interesting to note that all of the drugs are "more of the same", in other words they all repeat the same fate of previous drugs that have failed and continue to follow the same strategies mentioned before. This may be due to "herd theory", in which researchers continue to focus on what is known, as opposed to thinking out of the box and identifying novel drug targets and treatment strategies.

A last line approach for morbidly obese patients with a BMI of 30 and higher (but typically in the 35 and higher range) is bariatric surgery (Fisher and Schauer 2002). The most common of the bariatric surgeries in the United States is the Roux-en-Y gastric bypass surgery. This surgery has fallen into favor over the older vertical banded gastroplasty due to the fact it both limits the amount of food an individual can eat, and the amount of nutrients that can be extracted from the food. These surgeries are often used as a last resort after lifestyle changes and pharmacotherapies have both failed and/or are not possible (Fisher and Schauer 2002). 


\subsection{Need for new therapeutics in cognitive disorders and obesity.}

As shown, there are many current options clinicians have for the treatment of cognitive disorders; however most of these treatments are plagued by a plethora of side effects. In regards to anxiety, BZDs can cause addiction and sedation, and SSRIs/SNRIs may elicit nausea, weight change, paradoxical behavior, and suicidal thoughts. While the BZDs have incredible short term efficacy, they are inept at treating anxiety long term, and while the SSRIs/SNRIs and azapirones may be better at long term chronic use, they lack the ability to affect anxiety acutely. In addition, alternative therapies are safe and generally well tolerated but lack strong efficacy and consistent clinical evidence, and are difficult for patients to implement and subsequently have low compliance. Thus, there is yet to be a drug with the ability to modulate anxiety in both the short term and long term, without major side effects. Considering anxiety is the most common psychiatric disorder on the planet, the lack of an efficient therapeutic represents a considerable gap in the knowledge, and an aim for future drug development. While there has been progress made in the field of antidepressants, like anxiety, there is yet to be a panacea-like drug. The SSRIS/SNRIs have a long therapeutic lag, and are only effective in a small percentage of individuals. The older TCAs/MOIs are dampened by their dangerous side effects, and the therapies for TRD are often invasive and costly. TRD is very common, and as mentioned only about $30 \%$ of individuals respond to the first monotherapy. Thus, like anxiety, there is a strong need to develop novel antidepressants which are more effective with less side effects. AD therapeutics have had far less success than their anxiety and depression counterparts. While there are 5 currently approved therapies for $A D$, they lack significant clinical efficacy. Obesity therapeutics have met with even greater failure. Orlistat is the only currently approved 
pharmaceutical therapy for obesity in the United States, as the FDA has pulled many drugs off the markets due to safety concerns.

Due to the inherent drawbacks in all of the previously mentioned disorders, there remains a strong need to develop novel therapeutics to combat these diseases.

\subsection{Phosphodiesterase 4 and downstream signaling as a potential therapeutic for cognitive disorders, and obesity}

\subsubsection{Rationale for Phosphodiesterase-4 as a novel target for cognition and obesity. As}

previously mentioned, there is a strong need to develop novel therapeutics to combat cognitive disorders and obesity. However, before a therapeutic can be designed, a molecular target first needs to be identified. Phosphodiesterases (PDEs) may represent a future novel target for therapies aimed at combatting cognitive disorders and obesity. PDEs are a "super family" of 11 enzyme families (PDE1-11) responsible for the hydrolysis of the secondary messengers cyclic AMP (cAMP) and cyclic GMP (cGMP) (Zhang et al. 2002; Houslay and Adams 2003; Conti et al. 2003a; O'Donnell and Zhang 2004). The different families of PDEs are encoded through different genes and have unique protein structures, binding partners, tissue expression, and inhibitor specificities. In addition, PDE families can also be distinguished by their specificity for cyclic nucleotide degradation (Maurice et al. 2014). For example, some PDEs are capable of hydrolyzing both CAMP and CGMP (PDE1, 2, 3, 10, and 11), while others can only hydrolyze CAMP (PDE4, 7, and 8) or cGMP (PDE5, 6, and 9) (Menniti et al. 2006; Omori and Kotera 2007). Over the years PDEs have gained significant attention as novel drug targets (Zhang et al. 2006; Menniti et al. 2006; Richter et al. 2013). There are currently several FDA-approved PDE 
inhibitors: sildenafil (Viagra), a PDE5 inhibitor used for impotence (Smith et al. 2013),

roflumilast (Daxas) and apremilast (Otezla) , PDE4 inhibitors for the treatment of chronic obstructive pulmonary disease (COPD) (Pinner et al. 2012) and psoriatic arthritis (Varada et al. 2014) respectively, and cilostazol (Pletal), a PDE3 inhibitor used for intermittent claudication

(Cone et al. 1999). While there are 11 families of PDEs, PDE4 has garnered significant interest

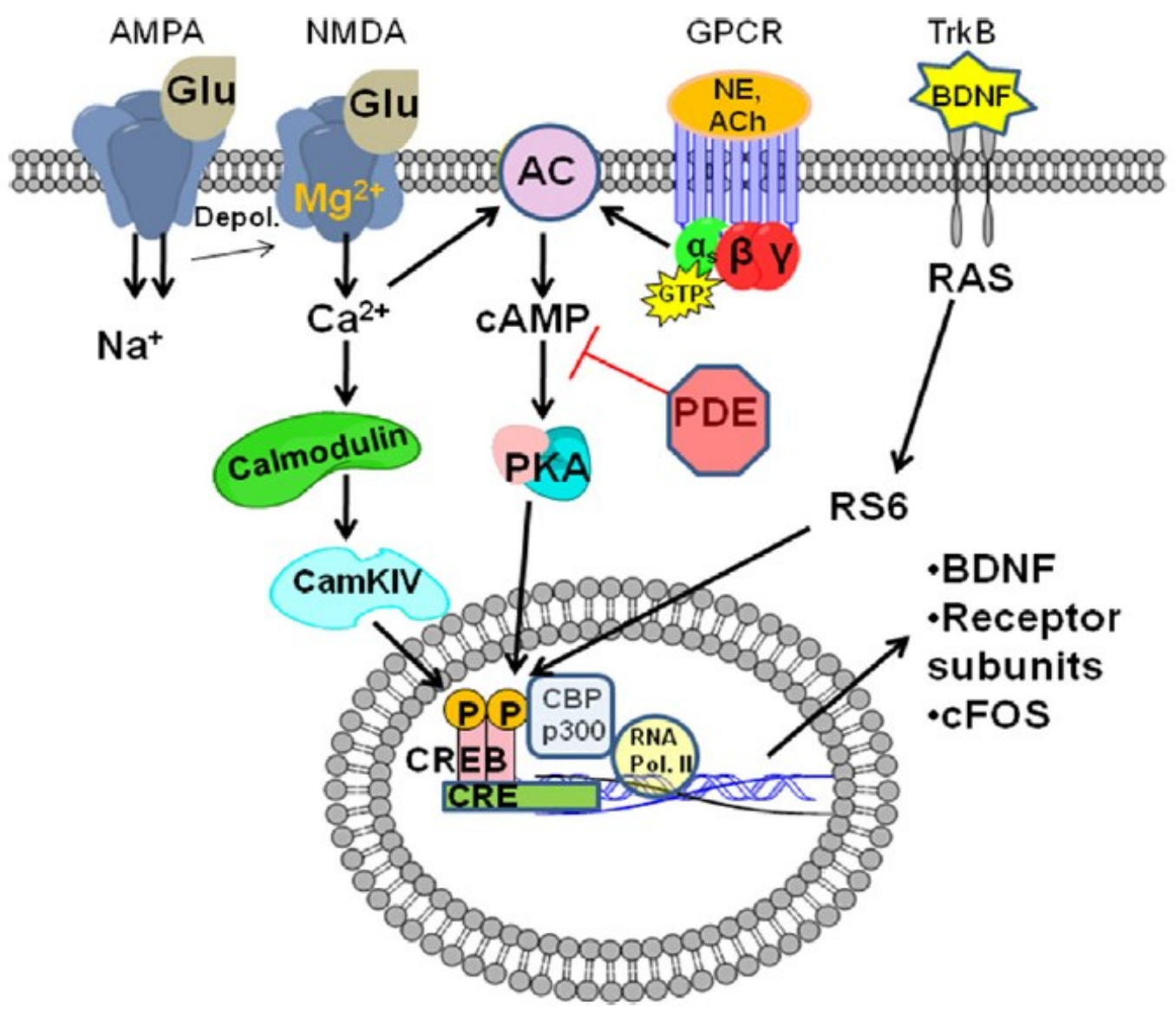

Fig. 1.1 - Simplified schematic of the CAMP/CREB signaling pathway. Abbreviations : Glutamate (Glu), 2-amino3-(3-hydroxy-5-methyl-isoxazol-4-yl) propanoic acid (AMPA), N-methyl-D-aspartate (NMDA) receptor, depolarization (depol.), rat-sarcoma protein (RAS), ribosomal s6 kinase (RS6), RNA polymerase II (RNA Pol II), CREB binding protein (CBP), G-protein coupled receptor signaling (GPCR), norepinephrine (NE), acetylcholine $(A C h)$, protein kinase $A(P K A)$, adenylyl cyclase $(A C)$, mitogen-activated protein-kinase (MAPK), receptor tyrosine kinase $b$ (TrkB), brain-derived neurotrophic-factor (BDNF) 
in cognitive and obesity research due to its high affinity for CAMP, and strong expression in the brain (Houslay and Adams 2003; Conti et al. 2003a; Zhang 2009), in particular regions involved with anxiety, depression, memory, and obesity, such as the amygdala, prefrontal cortex, hippocampus, nucleus accumbens, and hypothalamus (Cherry and Davis 1999; McPhee et al. 2001; Lakics et al. 2010; Johansson et al. 2012). Since the main function of PDE4 is the degradation of CAMP, the CAMP signaling pathway will be explored in the following section.

1.5.2. The cAMP-signaling pathway. CAMP and CGMP are the main second messengers of the cell, and are instrumental in orchestrating many molecular events and downstream cascades such as cAMP response element binding protein (CREB) (figure 1.1). Multiple pathways are capable of converging to produce the initiation of the cAMP pathway and initiate gene transcription. For standard G-protein coupled receptor signaling, binding of a ligand such as norepinephrine or acetylcholine to their respective Gas coupled receptor, liberates the as-subunit from the inhibitory $\beta$ and $\gamma$ subunitis. The activated $\alpha$ s-subunit then in turn activates adenylyl cyclase (AC). AC catalyzes the formation of CAMP from ATP in an energy dependent manner. Cyclic AMP activates protein kinase $\mathrm{A}(\mathrm{PKA})$ by binding to the regulatory subunits and relieving autoinhibition. Once activated, the catalytic units of PKA travel to the nucleus and phosphorylate the transcription factor CREB.

Glutamatergic signaling is also capable of activating the cAMP pathway through AMPA and NMDA receptors. Once the NMDA receptor is activated by AMPA induced depolarization, binding of glutamate, and expulsion of the magnesium ion, calcium flow through the NMDA receptor is capable of activating certain AC isoforms sensitive to calcium (I, III, VIII) which results in subsequent phosphorylation of CREB (Hanoune and Defer 2001). CREB is a very important 
transcription factor that has been implicated in many cognitive processes and also obesity, and could be a potential downstream target of altered PDE signaling (Hansen and Zhang 2013). In certain instances, tyrosine kinases involved with MAPK signaling can also activate the cAMPsignaling pathway (Gerits et al. 2008), and subsequent activation of CREB.

1.5.3. CREB and downstream trancription products. CREB is a $\sim 46 \mathrm{kDa}$ size protein residing in the nucleus that is capable of being activated by multiple pathways including CAMP/PKA-mediated signaling, $\mathrm{Ca2}^{+} / \mathrm{CamKII} / \mathrm{IV}$, or the MAPK/ERK pathway (Figure 1.1). There are two main subtypes of CREB (CREB1 and CREB2), and three different isoforms of CREB1 that are produced by alternative splicing ( $\alpha, \beta$, and $\delta$ ). Of interest for this discussion is CREB1, which will be referred to hereafter simply as CREB. CREB is a transcription factor (Montminy MR 1987) whose function in "learning" was first discovered in studies of Aplysia (Dash et al. 1990), and has now been extensively implicated in learning and memory (Bourtchuladze et al. 1994), long term potentiation (LTP) (Impey et al. 1996), neuro-protection (Ao et al. 2006), anxiety (Blundell and Adamec 2006; Adamec et al. 2011), depression (Muschamp et al. 2011), and obesity (Reusch et al. 2000; Qi et al. 2009).

Upon activation, CREB is phosphorylated at its serine 133 residue, dimerizes with another PCREB, binds its CAMP respone element (CRE) site on the promoter DNA via a leucine zipper domain, and then recruits several co-activator proteins to assist with transcription. Among the co-activator proteins recruited are CREB binding protein (CBP), p300, and RNA polymerase II. CBP and p300 both contain histone acetyl-transferase ability, which relaxes the chromatin structure from histones, making it more readily available for transcription by Pol II 
(Johannessen et al. 2004; Carlezon et al. 2005). In regards to CREB kinetics, it is important to note that CREB may already be dimerized and bound to CRE sites under basal conditions, and simply becomes activated during phosphorylation. This is a controversial area and still needs further clarification (Cha-molstad et al. 2004).

There are also several proteins worth mentioning that are capable of modifying CREB activity. The phosphatases PP1 and PP2A are both involved with negative regulation of CREB, and have been shown to terminate its signaling (Wadzinski et al. 1993; Bito et al. 1996). In addition, the inducible cAMP early repressor (ICER), which is derived from the CAMP-responsive element modulator (CREM) gene via an alternative intrinsic promoter, is capable of inhibiting CREB activity by binding to the CRE domain. ICER is able to do this without activation of transcription because it lacks the activation and kinase inducible domains present on CREB, thus ICER competes with CREB for the CRE sites and acts as a negative regulator. Interestingly, the alternative intrinsic promoter for ICER contains a CRE site, and ICER transcription itself is driven by pCREB activation. Thus CREB is able to succinctly regulate its own activity by driving transcription of its repressors (Lonze and Ginty 2002; Borlikova and Endo 2009).

The CREB transcriptome has currently been identified at approximately 1,663 CRE targets in humans and 1,349 possible binding sites in mice, though the actual functional transcriptome differs drastically depending on the tissue and cell type (Conkright et al. 2003). Some important transcripts downstream of CREB include brain derived neurotrophic factor (BDNF) and c-FOS. BDNF is a neurotrophic molecule that has extensively been linked to LTP (Patterson et al. 1996; Korte et al. 1996; Figurov et al. 1996; Korte et al. 1998; Xu et al. 2000; 
Patterson et al. 2001) learning and memory (Hall et al. 2000), anxiety (Lakshminarasimhan and Chattarji 2012) and obesity (Schwartz and Mobbs 2012). BDNF binds to the TrkB receptor (Xu et al. 2000) and can be released from neurons upon stimulation (Androutsellis-Theotokis et al. 1996; Kojima et al. 2001). The relationship between CREB and BDNF is cyclical; BDNF activates CREB transcription (Finkbeiner et al. 1997), which in turn increases BDNF transcription via the CRE promoter (Tao et al. 1998; Shieh and Ghosh 1999).

\subsubsection{Background of PDE4.}

The PDE4 enzyme is

unique from other PDEs

in that it contains two

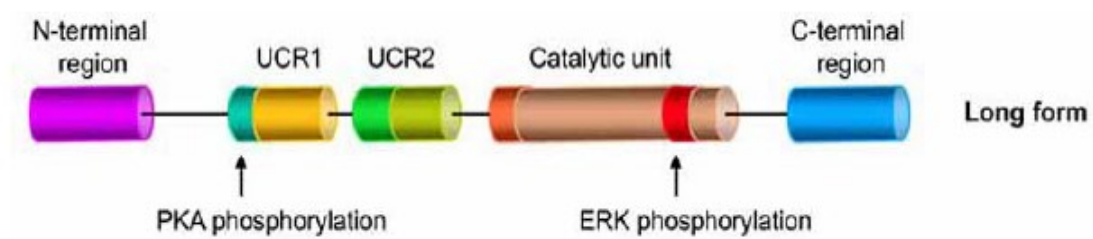

upstream conserved

regions (UCRs) termed

UCR1 and UCR2 located

towards the $\mathrm{N}^{\prime}$ -

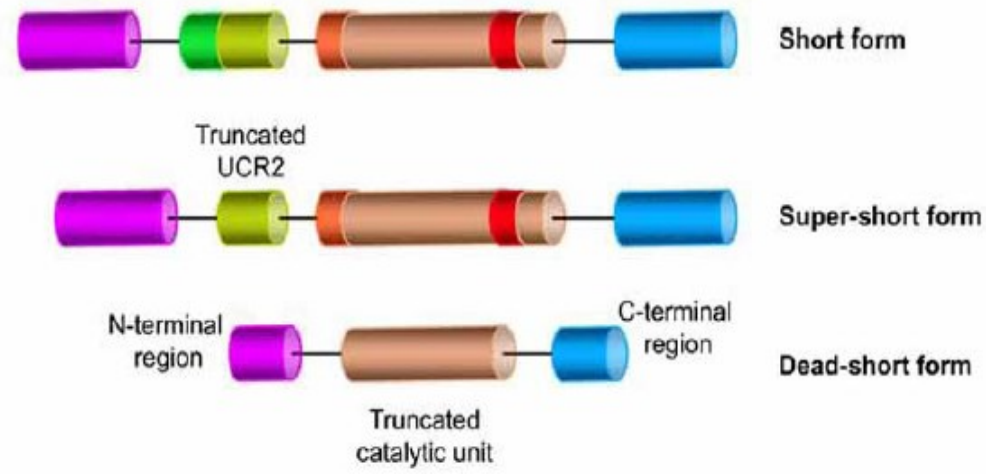

terminus region. These UCR

Figure 1.2. PDE4 protein-domain structure, (taken from Zhang 2009, Curr. Pharm. Design)

regions play an important

regulatory role in the enzyme, and have also been shown to be very important for intracellular targeting (Jang et al. 2010). Downstream towards the $C^{\prime}$ - terminus lies the catalytic domain of the PDE4 enzyme which is responsible for the hydrolysis of cAMP to 5'-AMP (Saldou et al. 1998; Houslay and Adams 2003; Conti et al. 2003a; Zhang 2009; Houslay 2010). 
PDE4 was first biochemically characterized as an enzyme capable of cAMP phosphodiesterase activity from rat liver plasma membranes (Marchmont and Houslay 1980); however, its involvement in cognition and behavior was first identified through mutations in the Drosophila melanogaster dunce PDE4D gene which led to deficient cAMP-specific PDE activity and abnormal memory (Kauvar 1982; Qui et al. 1991). PDE4 was later discovered in both rats (Davis et al. 1989; Swinnen et al. 1989) and humans (Bolger et al. 1993) to consist of a small family of similar enzymes encoded through 4 different genes, which are now referred to as PDE4-A, B, C, and D (Reeves et al. 1987; Wang et al. 1997; Houslay and Adams 2003; O'Donnell and Zhang 2004). These PDE4 subtypes are not expressed equally throughout the body, as PDE4C has been shown to be largely deficient in the brain (Cherry and Davis 1999; Perez-Torres et al. 2000; Lakics et al. 2010). Thus, in regards to cognitive research PDE4-A, B, and $D$ are the predominant subtypes which are now focused on (Zhang 2009).

In addition to the individual PDE4 subtypes, there are also multiple PDE4 splice variants, which can be produced from alternative promoters and splicing. Up to 25 different PDE4 splice variants have been identified to date, including 6 PDE4A splice variants, 5 PDE4B variants, 3 PDE4C variants, and 11 PDE4D variants (Bolger et al. 1996; Bolger et al. 1997; Huston et al. 1997; Sullivan et al. 1998; Rena et al. 2001; Miró et al. 2002; Shepherd et al. 2003; Bolger et al. 2003b; Wallace et al. 2005; Richter et al. 2005; Chandrasekaran et al. 2008; Mackenzie et al. 2008; Zhang 2009). This alternative splicing results in the formation of different length PDE4s, which have come to be classified as long form, short form, super-short form, and dead-short form (figure 1.2). Long-form PDEs are full length and contain no truncation, short forms lack a UCR1 region, super-short forms lack a UCR1 and have a truncated UCR2, and dead-short lack 
both UCR regions and also contain a truncated catalytic domain (O'Donnell and Zhang 2004; Zhang 2009; Richter et al. 2013). Interestingly, this alternative splicing has been shown to be increased in mammals and humans, suggesting that the increasing complexity of nonredundant PDE4 variants observed in humans parallels the increasing levels of brain function (Johnson et al. 2010).

1.5.5. History of PDE4 as a cognitive therapeutic: pitfalls, promises, and layers of complexity. The idea of using PDE4 as a target for psychiatric illness is not a novel one. Since PDE4 was first discovered, it was found shortly thereafter that the broad PDE4 inhibitor rolipram significantly improved memory (Randt et al. 1982; Imanishi et al. 1997; Egawa et al. 1997; Barad et al. 1998), and reversed depressive-like behavior in rodents (Wachtel 1983; Wachtel and Schneider 1986). Because of these initial findings, rolipram made it to clinical trials as a potential antidepressant and showed both promising (Zeller et al. 1984; Guiot-Goffioul et al. 1987; Laux et al. 1988) and inconsistent results (Bertolino et al. 1988). However, it was soon discovered that rolipram had a major flaw; several studies began confirming that rolipram induced severe nausea and emesis (Hebenstreit et al. 1989; Scott et al. 1991), and the idea of rolipram as a therapeutic for depression began to fade. Interestingly, the PDE4 inhibitor roflumilast is currently approved for COPD, and able to avoid the side effects plagued by rolipram. The main reason for the lack of side effects however is most likely due to the low blood-brain barrier permeability of roflumilast, indicating that the area postrema is never activated.

The failure of rolipram in clinical trials leads to an important question, why was rolipram destined to fail? It has since been discovered that PDE4 is highly expressed in the area postrema (Cherry and Davis 1999) which is the emetic center of the brain (Mori et al. 2010). 
Inhibition of the PDE4 in this region "activates" the area postrema, and produces the emetic

response typical of rolipram (Heaslip and Evans 1995; Robichaud et al. 2001; Richter et al.

2013).

The failed attempts of rolipram in clinical trials (and subsequent characterization of the

\section{Layers of Phosphodiesterase 4 Complexity}

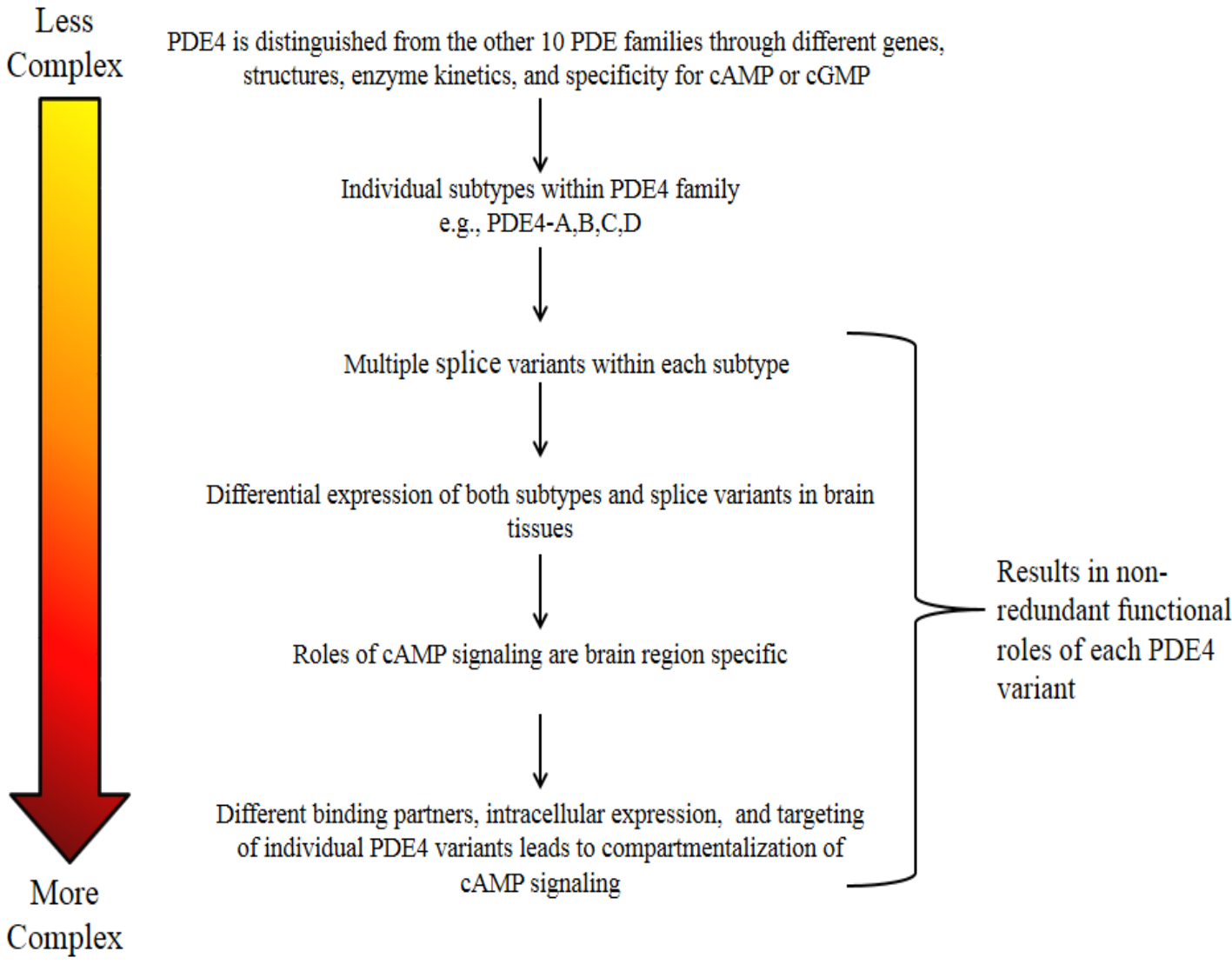

Figure 1.3. Layers of PDE4 complexity. When considering PDE4 as a therapeutic target, there are varying layers of complexity that need to be overcome. First, PDE4 is expressed in many regions in the periphery in addition to the CNS that could lead to off target side effects. PDE4 also has multiple subtypes and splice variants that have differential expression in brain tissues. Complicating this further is the fact that cAMP signaling has different roles in various brain regions. Finally, within these brain regions and neurons, the multiple splice variants have different binding partners, and different targeting and intracellular expression which leads to compartmentalization of cAMP signaling. These multiple "layers of PDE4 complexity" could result in non-redundant functional roles for each PDE4 variant which makes broad PDE4 inhibition as a future therapeutic a difficult therapeutic. PDE4 subtype and splice-variant specific therapeutics are more likely to be successful in the future, however much more work is still needed to develop these therapies.

mechanisms) has shed light onto the "layers of PDE4 complexity" (Figure 1.3). The first 
complexity of PDE4 is that it has multiple subtypes as described previously (PDE4A, B, C, and D). In addition, these subtypes are encoded through different genes, PDE4 then has multiple splice variants within each subtype produced through alternative splicing and promoters. These individual splice variants of PDE4 are then differentially expressed throughout the body, both peripherally and centrally, as well as in discrete regions even within the brain. The level of complexity is increased by the fact that CAMP has various functions in different regions of the body. Lastly, even within discrete brain regions and individual neurons themselves, PDE4 splice variants have different binding partners and intracellular expression which leads to unique targeting of the splice variants, and subsequent compartmentalization or "pools" of cAMP that have contrasting functions (Houslay and Adams 2003; Baillie 2009; Blackman et al. 2011). Because of this incredible complexity, focus in the PDE4 field has shifted from PDE4 in general, to the characterization and targeting of specific PDE4 subtypes in hopes of circumventing the side effects of broad PDE4 inhibition. The PDE4 subtypes and splice variants will be talked about in the following sections.

1.5.4. PDE4D. One of the main PDE4 subtypes is PDE4D, which has the most splice variants of the PDE4 family, and is the most studied. PDE4D currently has 11 splice variants termed PDE4D1-11; PDE4D1/2 are short forms, PDE4D6 is super short, and the remaining splice variants are all full length PDE4 proteins (Bolger et al. 1997; Conti et al. 2003a; O'Donnell and Zhang 2004; Cheung et al. 2007; Houslay et al. 2007; Chandrasekaran et al. 2008; Lynex et al. 2008; Zhang 2009; Maurice et al. 2014).

PDE4D has a diffuse pattern of expression throughout the brain, with noticeable expression in the hippocampus, cortex, thalamus, area postrema, periaqueductal grey, brain 
stem, and cerebellum (Engels et al. 1995; Cherry and Davis 1999; Perez-Torres et al. 2000; McPhee et al. 2001; D'Sa et al. 2005; Lakics et al. 2010; Johansson et al. 2012; Kelly et al. 2014). In addition, PDE4D also has specific binding partners such as myomegalin protein (Verde et al. 2001), beta arrestins (Baillie et al. 2003; Bolger et al. 2003a; Lynch et al. 2005; Baillie et al. 2007; Lynch et al. 2007; Li et al. 2009a), RACK1 (Conti et al. 2003a), SH3-domain regions (Beard et al. 1999) and the A-Kinase anchoring proteins (AKAPS), Erk, and exchange protein directly activated by cAMP (EPAC) (Dodge et al. 2001; Dodge-Kafka et al. 2005).

PDE4D has also been extensively studied using PDE4D KO mice designed by homolgous recombination (Jin et al. 2005b) and through viral manipulation. Through these techniques it has been found that PDE4D KO mice exhibit improved memory (Li et al. 2011), increased synaptic long-term potentiation (LTP) (Rutten et al. 2008), reduced levels of depressive-like behavior (Zhang et al. 2002);, elevated neurogenesis (Zhang et al. 2002; Li et al. 2011), and impaired growth and fertility (Jin et al. 1999) This is supported by studies showing that RNAi or inhibitors with relatively high selectivity for PDE4D also produce memory-enhancing effects (Burgin et al. 2010; Li et al. 2011; Bruno et al. 2011), reversed memory deficits induced by beta-amyloid peptide 1-42 (Zhang et al. 2014, and/or antidepressant activity (Wang et al. 2013). However, PDE4D KO also causes emetic-like behavior, the major side effect of PDE4 inhibitors (Robichaud et al. 2002a; Robichaud et al. 2002b). These studies have greatly illustrated the role that PDE4D plays in behavior. We are now aware that the PDE4D subtype plays a strong role in memory, probably due to its high expression in the hippocampus. PDE4D is also one of the major subtypes in the mediation of depressive-like behavior, as mice deficient in PDE4D were less sensitive to the anti-depressant effect of rolipram. Unfortunately, the PDE4D subtype is also the main 
mediator of the emetic side effects seen with rolipram. Upon further evaluation this data makes sense, since PDE4D has the highest expression of any of the PDE4 subtypes in the area postrema (Cherry and Davis 1999; Mori et al. 2010). Recently, PDE4D specific allosteric modulators have also become avaiable which only "partially" inhibit PDE4D; this study found that they were able to reduce the emetic effects of PDE4D inhibition as measured through duration of anesthesia induced by ketamine/xylazine (Robichaud et al. 2002a; Robichaud et al. 2002b) but maintian the nootropic effect (Burgin et al. 2010). While it was previously very difficult to make a PDE4 subtype-specific inhibitor, $x$-ray structure of the PDE4 proteins (Wang et al. 2007a) revealed a phenylalanine-196 residue that was only present on PDE4D, which was able to be targeted by allosteric modulators (Burgin et al. 2010). The memory enhancing effects of PDE4D dysruption have also been supported by more recent relatively selective PDE4D inhibitors (Bruno et al. 2011; Sierksma et al. 2013)

PDE4D single nucleotide polymorphisms (SNP) have also been associated with stroke (He et al. 2013; Liu et al. 2013b; Liu et al. 2013a; Heyer et al. 2013), however the results have been inconsistent (Shao et al. 2014). One study has confirmed that the PDE4D SNP83 is higher in Asian and Chinese populations, but not among Caucasians by conducting a meta-analysis with a much larger sample size (Yan et al. 2014). PDE4D mutations have also been associated with skeletal dysplasia and intellectual disability (Lindstrand et al. 2014).

While we are beginning to understand the role PDE4D plays in behavior, much additional research is needed to further characterize the role the PDE4D subtype, and more importantly, its splice variants, play in behavior and cognition. 
1.5.5. PDE4B. PDE4B has 5 splice variants identified PDE4B1-5; PDE4B1, 3, and 4 are long isoforms, PDE4B2 is short, and PDE4B5 is super short (Bolger et al. 1997; Conti et al. 2003a; O'Donnell and Zhang 2004; Cheung et al. 2007; Houslay et al. 2007; Chandrasekaran et al. 2008; Lynex et al. 2008; Zhang 2009).

While most of the PDE4 subtype expression patterns overlap a good bit and vary with different studies, PDE4B is unique in the fact that it is consistently the highest PDE4 subtype expressed in the striatum, globus-pallidus, nucleus accumbens, hypothalamus, and amygdala (Engels et al. 1995; Cherry and Davis 1999; Perez-Torres et al. 2000; McPhee et al. 2001; D’Sa et al. 2005; Lakics et al. 2010; Johansson et al. 2012; Kelly et al. 2014). PDE4B is also unique in that mutations in PDE4B are strongly believed to play a role in schizophrenia through its interaction with disrupted in schizophrenia 1 protein (DISC1) (Millar et al. 2005; Fatemi et al. 2008; Numata et al. 2008; Numata et al. 2009; Kähler et al. 2010). Interestingly, it was observed that this PDE4B mutation results in a decrease of PDE4B expression (Fatemi et al. 2008).

PDE4B KO mice studies have shown that PDE4B is the main PDE4 subtype responsible for the release of tumor necrosis factor- $\alpha$ (TNF- $\alpha$ ) in response to lipopolysaccharide injections (LPS). In particular, these studies found that PDE4B KO reduced the amount of TNF- $\alpha$ released in response to LPS, while PDE4A and PDE4D did not play a role (Jin and Conti 2002; Jin et al. 2005a). Thus, it appears that PDE4B may be one of the main PDE4 subtypes involved with mediation of the immune response, as PDE4B has also been shown to be involved with Amyloid beta induced microglia activation (Sebastiani et al. 2006). Additional PDE4B KO studies have shown that PDE4B $\mathrm{KO}$ is anxiogenic and increases corticosterone levels, and also decreases depressive-like behavior in mice (Zhang et al. 2008) 
Until very recently, PDE4B specific inhibitors were not available. However, Burgin and colleagues have recently designed PDE4B allosteric modulators (Burgin et al. 2010) and a separate group has made PDE4B inhibitors using a different approach by targeting the Leu674 residue in PDE4B region CR3 (Fox et al. 2014).

While we are beginning to understand the role PDE4B plays in behavior, additional research is needed to further characterize the role that the PDE4B subtype, and more importantly, its splice variants, play in behavior and cognition.

1.5.6. PDE4A. The PDE4A subtype has 6 splice variants; PDE4A1 is short (Sullivan et al. 1998), PDE4A7 is the dead-short form (Horton et al. 1995; Johnston et al. 2004), and PDE4A5 (McPhee et al. 1995), PDE4A8 (Bolger et al. 1996), PDE4A10 (Rena et al. 2001), and PDE4A11 (Wallace et al. 2005) are long forms (Bolger et al. 1997; Owens et al. 1997; Conti et al. 2003a; O'Donnell and Zhang 2004; Cheung et al. 2007; Houslay et al. 2007; Chandrasekaran et al. 2008; Lynex et al. 2008; Zhang 2009). The broad PDE4A subtype has been found to be strongly expressed in the olfactory bulbs, deep layers of the cortex, amygdala, hypothalamus, hippocampus, piriform cortex, and cerebellum (Engels et al. 1995; Cherry and Davis 1999; Perez-Torres et al. 2000; McPhee et al. 2001; D’Sa et al. 2005; Lakics et al. 2010; Johansson et al. 2012; Kelly et al. 2014).

Interestingly, PDE4A is one of the few PDE4 subtypes where differential splice-variant expression patterns have been looked at. McPhee and colleagues found that the long PDE4A5/10 isoforms were present throughout most of the cortex, CA1, and CA2 in overlapping patterns, however, PDE4A10 was present in the islands of Calleja while PDE4A1 and PDE4A5 were not. In addition, PDE4A5 and PDEA10 were present in the medial nucleus of the amygdala, while PDE4A1 
expression was absent. PDE4A1 had a different expression pattern than the longer isoforms, with very strong expression in the glomerular layer of the olfactory bulbs, CA3 of the hippocampus, the cerebellum, and low staining in the brain stem (McPhee et al. 2001). D'Sa and colleagues also looked at PDE4A expression, and observed slightly different patterns. Specifically, they saw that PDE4A1 was high in the medial septum, diagonal band, olfactory system, hippocampus and cerebellum; PDE4A5 was highly expressed in the olfactory nuclei, deep cortical layers, and the DG and CA1 of the hippocampus; PDE4A10 was highly expressed in the DG and CA1, and PDE4A8 expression was absent in the brain (D'Sa et al. 2005)

PDE4A1 has also been found to be unique, in that it is inserted into lipid bilayers through a TAPAS-1 (tryptophan anchoring phosphatidic acid selective-binding domain 1); these bilayers are typically found on golgi that traffic out to the membrane (Baillie et al. 2002). PDE4A5 is unique from the remaining subtypes in that it can interact with $\mathrm{SH} 3$ domains on proteins through its $\mathrm{N}^{\prime}$ Terminal region (O'Connell et al. 1996). This SH3 binding plays a significant role in the targeting of PDE4A5, which has been found to be expressed in perinuclear regions, and also within membrane ruffles (Beard et al. 2002). This expression pattern of PDE4A5 has been shown to be disrupted in apoptotic cells by cleavage of caspase 3 at its $\mathrm{SH} 3$ binding sites, and overexpression of PDE4A5 protects against apoptosis (Huston et al. 2000). Another unique trait of PDE4A5 is that it can bind to an immunophilin known as XAP2 which partially inhibits the enzyme up to $60 \%$, and increases its sensitivity to rolipram (Bolger et al. 2003b). Interestingly, PDE4A can also be activated by the P70S6 kinase in adipocytes during adipogenesis, which suggests it could play a role in obesity (MacKenzie et al. 1998). Lastly, PDE4A5 is the only member of the PDE4A family which can be phosphorylated by MAPK which tends to attenuate 
its activation by PKA (MacKenzie et al. 2011). The PDE4A7 and PDE4A8 splice variants are exclusively found in the testis in rodents, however in humans PDE4A8 has undergone an evolutionary change which causes it to be expressed in the brain (Mackenzie et al. 2008).

While there has been some progress on the functional roles of PDE4B and D in behavior, the role that PDE4A plays in behavior is largely unknown. While PDE4A KO mice have been examined for olfaction, no changes were found and behavior was not tested (Cygnar and Zhao 2009). Interestingly, antidepressant treatment has been shown to increase PDE4A expression in limbic and cortical regions (Ye et al. 2000; D'Sa et al. 2005). It has also been shown that PDE4A activity is significantly reduced in obese individuals (Omar et al. 2011) and mice (Greene et al. 2009), suggesting that altered PDE4A activity could be a mechanism for obesity-related pathology. Additional evidence has shown that sleep-deprivation-induced memory deficits correlate with impaired cAMP signaling, increased PDE4A5 expression, and elevated PDE4 activity (Vecsey et al. 2009). These studies, along with the fact that PDE4A expression is observed in the prefrontal cortex, hypothalamus, amygdala, and striatum, (memory, anxiety, depression, and obesity-related brain regions) indicate that PDE4A could play a significant role in the mediation of anxiety, memory, depression, and/or obesity. This gap in the knowledge represents a significant opportunity for study, as the characterization of PDE4A in these states could have profound impacts on the development of future drug targets, and the PDE4 field; this led to the purpose of this dissertation and following studies, which will be covered in the next section.

\subsection{Summary and Objectives}

The progress in developing PDE4 targeted therapeutics for cognitive disorders has been unsuccessful, and been stagnant over much of recent history. This is mostly due to the strong 
side effects associated with broad PDE4 inhibition such as nausea and emesis. In addition, pharmaceutical treatments for obesity have largely failed, and recently many of the formerly FDA approved obesolytics were pulled due to safety concerns, leaving Orlistat as the only FDA approved therapy.

Recently, the specific targeting of individual PDE4 subtypes or splice variants has begun to shed light into their incredible complexity and differential functional roles. Through the use of KO mice and viral manipulation, it has been shown that PDE4D is involved with the main emetic response of rolipram, and is also involved with antidepressant-like behavior and memory.

PDE4B KO mice have shown an anxiogenic profile, elevated corticosterone, decreased immune response, and decreased depressive-like behavior. However, the functional role that PDE4A plays in behavior and obesity is still unknown.

As mentioned, anxiety, depression, $A D$, and obesity are considerable public health threats in need of novel therapeutics with high efficacy and low side effects. Interestingly, it has also been shown that depression, anxiety, and obesity are highly co-morbid. However, the causal relationship between these three is still currently unknown. Due to the fact PDE4A is strongly expressed in regions of the brain important for anxiety, depression, $A D$, and obesity, it is possible that PDE4A could be a novel target for future therapeutics aimed at combatting these diseases. Thus the objective of this study was to investigate the role that PDE4A plays in behavior and obesity through the following aims:

\section{Characterize the functional role that PDE4A plays in behavior through the use of PDE4A KO mice.}


PDE4A KO mice will be generated through homologous recombination and provided by our collaborator Dr. Marco Conti of UCSF. The role of PDE4A in behavior will then be characterized through a large battery of behavioral tests looking at activity, nociception, visual acuity, social interaction, memory, anxiety, and depression. In addition, biochemical tests will also be performed to look at hormone levels in the body, as well as molecular changes to the CAMP/PDE signaling pathway.

2. To Characterize the functional role that PDE4A plays in obesity through the use of PDE4A KO mice

The relationship between obesity and PDE4A will be examined through PDE4A KO mice. PDE4A KO mice (see aim 1) will be carefully monitored for body weight, changes in activity, food consumption, and differences in metabolic hormones.

Our central hypothesis for the study is that PDE4A KO will be Nootropic, obesogenic, and anxiogenic.

\subsection{Contributions}

Portions of this background were taken from a previously published review (Hansen and Zhang 2013). 
Chapter 2

\section{PDE4A KO and behavior}




\subsection{Introduction}

Cognitive disorders such as anxiety, depression, and pathological/non pathological memory loss are growing pandemics in this country that represent considerable public health threats. From this list of public mental health threats, anxiety disorders in particular are the most common affecting upwards of $20 \%$ of the population and costing the American economy over 50 billion dollars a year (Greenberg et al. 1999).

Anxiety has been described as "a psychological, physiological, and behavioral state induced in animals and humans by a threat to well-being and survival, either actual or potential" (Steimer 2011). Because of this, it can be argued that anxiogenic behaviors were essential to the survival of species, and probably evolved as an adaptive trait in early animals. However, in pathological anxiety there seems to be a dysadaptation, in which animals or humans are unable to appropriately adjust to varying degrees of stress whether actual or perceived. While there are treatments for anxiety such as therapy (cognitive behavioral therapy, exposure therapy), medications (benzodiazepines, selective serotonin reuptake inhibitors-SSRIs), and complementary alternative therapies (yoga, acupuncture), anxiety still remains a prominent problem. Although anxiolytics are effective in alleviating anxiety, they come so with a price. Due to their general sedative properties benzodiazepines can result in severe drowsiness, leading to problems of impaired coordination and blunted attention. This side effect becomes increasingly problematic when related to employment and the work force, as anxiolytics can significantly impair the ability of a person to effectively maintain the level of concentration needed to maintain workplace composure. Benzodiazepines can even result in severe addiction, nausea, personality changes, aggression, depression, memory impairment, and paradoxical reactions 
resulting in increased anxiety. In addition, SSRIs have a list of unwanted side effects such as severe nauseas and diarrhea, rapid weight changes, insomnia, renal failure, cognitive disorders, and even increased risk of depression and suicide in certain individuals. SSRI use is also dampened by the fact that they may take weeks or months to take effect, and are ineffective in a large percentage of individuals (Bandelow et al. 2013).

The plethora of side effects induced by anxiolytics is most likely due to the very broad mechanism by which they act. Benzodiazepines work by enhancing GABAergic transmission; while SSRIs work by inhibiting the serotonin transporter and globally increasing the amount of extracellular serotonin. In addition to being expressed in anxiety-related brain regions, GABA and serotonin receptors are highly expressed throughout the body which is involved in their many off target side effects (Akinci and Schofield 1999; Barnes and Neumaier 2011). Thus, anxiolytics are limited by their inherent lack of specificity to anxiogenic brain regions and the broad mechanisms by which they achieve their effects. Because of this, there is a strong need to develop novel anxiolytics that are able to act in a more specific manner with fewer side effects than those that currently hinder modern anxiolytics. One possible candidate target for the development of new therapies is the phosphodiesterase (PDE) super family (Menniti et al. 2006; Zhang 2009).

PDEs are a "super family" of 11 smaller families of enzymes responsible for the hydrolysis of the secondary messengers CAMP and cGMP (Zhang et al. 2002; Houslay and Adams 2003; Conti et al. 2003a; O’Donnell and Zhang 2004). The different families of PDEs are encoded through different genes and have unique protein structures, binding partners, tissue expression, and inhibitor specificities. In addition, PDE families can also be distinguished by their specificity for 
cyclic nucleotide degradation. For example, some PDEs are capable of hydrolyzing both cGMP and cAMP (PDEs 1,2,3,10,11), some PDEs can only hydrolyze cGMP (PDEs 5,6,9), while others can only hydrolyze cAMP (PDEs 4,7,8) (Menniti et al. 2006; Omori and Kotera 2007).

Over the years PDEs have gained significant interest as novel drug targets due to their vast differences in tissue expression (which gives them increased specificity), and also the power that they control over intracellular signaling (Zhang et al. 2006). There are currently several classes of FDA approved PDE inhibitors such as Sildenafil, which is a PDE5 inhibitor that works on cGMP levels in the corpus cavernosum to increase smooth muscle dilation (Smith et al. 2013), and PDE4 inhibitors such as roflumilast for the treatment of chronic obstructive pulmonary disease (COPD) (Pinner et al. 2012). PDE4 inhibitors may also have important actions in areas other than the periphery. There has been much investigation into the field of PDE4 and cognition mediated by the central nervous system.

PDE4 is of particular interest with relation to cognitive disorders due to its high affinity for cAMP and strong expression in multiple regions such as the hippocampus, amygdala, and nucleus accumbens which are important for memory, anxiety, and depression, respectively (Cherry and Davis 1999; McPhee et al. 2001). PDE4 and its subtypes are distinguished from the 11 other families of PDEs by their unique protein structure. This structure consists of two regions termed UCR1 and UCR2 (upstream conserved regions) towards the $\mathrm{N}-{ }^{-}$terminus of the enzyme. Downstream towards the C-' terminus is the other main domain of the protein consisting of the catalytic unit. In addition, alternative splicing can result in the formation of different length PDE4s, which can be classified as: long form, short form, super short form, and dead-short form (Conti et al. 2003b; Houslay and Adams 2003). Long form PDE4s have all the 
domains intact, short forms have a missing UCR1 region, super short forms have a missing UCR1 and truncated UCR2, and dead-short forms are missing both UCR regions and also have a truncated catalytic unit rendering them functionally silent (O'Donnell and Zhang 2004; Zhang 2009).

PDE4 has four subtypes (A, B, C, and D), of which only PDE4C is not strongly expressed in the brain (Cherry and Davis 1999). Because of this, the main focus of PDE4 in regards to cognition has been on subtypes $A, B$, and $D$. These subtypes are distinguished from one another by the fact they are encoded by separate genes and contain different $N^{\prime}$ terminus domains(Conti et al. 2003b; Houslay and Adams 2003; O’Donnell and Zhang 2004; Zhang 2009). In addition, the level of complexity is increased with multiple splice variants belonging to each subtype due to alternative splicing and multiple promoter sites.

Until recently, the functional role of PDE4 subtypes in the CNS was unknown. However, through the use of gene knockout (KO) mice, viral vector-mediated RNA interference (RNAi), and recent PDE4-subtype selective inhibitors, we have now been able to study the functional role of individual PDE4 subtypes in the CNS. It has been found that PDE4D KO or inhibition improves memory (Burgin et al. 2010; Li et al. 2011; Bruno et al. 2011), increases synaptic LTP (Rutten et al. 2008), reduces levels of depressive-like behavior ( Zhang et al. 2002; Wang et al. 2013), and increases adult neurogenesis (Li et al. 2011). However, PDE4D KO also causes emetic-like behavior, the major side effect of PDE4 inhibitors (Robichaud et al. 2002a; Robichaud et al. 2002b). In addition, PDE4B KO mice exhibit anxiety-like behavior in multiple behavioral tests and also show elevated plasma corticosterone (Zhang et al. 2008). While 
progress has been made in our understanding of PDE4B and PDE4D, the functional role that PDE4A plays in the mediation of behavior has yet to be studied.

The PDE4A subtype can be further broken down into six splice variants: PDE4A1, A5, A7, $A 8, A 10$ and $A 11$. These splice variants are comprised of different lengths (A1=short form, A7=dead form, A5, A8, A10, A11=long forms), and are also expressed differentially in the body. (McPhee et al. 1995; Sullivan et al. 1998; Richter et al. 2001; McPhee et al. 2001; Cherry and Pho 2002; Bolger et al. 2003b; D'Sa et al. 2005). The expression pattern of PDE4A splice variants is complex, and can vary from rodent to human. For example, the PDE4A8 variant is found primarily in the testis of rodents, while in humans it has gained an extended $\mathrm{N}-{ }^{\prime}$ terminal region and is also expressed in skeletal muscle and the brain (Mackenzie et al. 2008). PDE4A7 is widely expressed, but is functionally silent (Johnston et al. 2004) . The long isoforms PDE4A5/10 are found in most of the same brain regions such as the striatum, thalamus, hippocampus (CA1, CA2, DG), medial nucleus of amygdala, olfactory nuclei, and deep cortical layers; however, one noted exception is expression of PDE4A10 in the islands of Calleja in which PDE4A5 expression is absent. PDE4A1 exhibits a similar expression profile, but it is not expressed in the amygdala, while it was very strongly expressed in the cerebellum where most of the longer isoforms are absent (McPhee et al. 2001). While these few studies are starting to characterize the expression of PDE4A splice variants and function at the molecular level, much more work is needed to characterize the functional role of PDE4A in mediating cognition and behavior. Thus the goal of this study was to characterize the role that PDE4A plays in cognition through the use of PDE4A KO mice and a battery of behavioral tests. 


\subsection{Materials and Methods.}

\section{Animals}

All procedures were approved by the Animal Care and Use Committee of West Virginia University Health Sciences Center and performed according to the recommendations of the NIH Guide for the Care and Use of Laboratory Animals $7^{\text {th }}$ edition (NIH Publications No. 80-23, revised 1996). Adult male PDE4A KO mice on C57 background were generated by homologous recombination and heterozygote breeding as described previously (Jin et al. 1999; Jin and Conti 2002; Ariga et al. 2004; Jin et al. 2005a; Bruss et al. 2008; Jin et al. 2010). In brief, the PDE4A gene was disrupted through replacement of a $3 \mathrm{~kb}$ region encoding exons 10-14 using a PGKhrpt cassette in the reversed orientation via homologous recombination. These PDE4A KO mice are commercially available through the Mutant Mouse Regional Resource Centers which also provides additional details on their generation (MMRRC; http://www.mmrrc.org; Stock number 034793-UCD). Age-matched (4-8 months) wild-type (WT) mice (C57BL/6J) of the same background were used in this study. All genotyping was confirmed by Southern blot analysis with tissues obtained via tail clipping and subsequent detection of genomic DNA. Animals were housed in groups of 2-5 per cage (Allentown XJ cage, 12.5" length x 6" width x 5" height, 75 square inches of floor space) in a light (12 h on, $12 \mathrm{~h}$ off; lights on at $0600 \mathrm{~h}$ ) and temperature controlled room $\left(22-23^{\circ} \mathrm{C}\right)$. Water and food were available ad libitum.

\section{Behavioral Paradigm}

Two batches of adult WT and PDE4A KO mice were used to investigate the role of PDE4A on behavior and biochemistry. Batch 1 consisted of two groups of WT $(n=9)$ and PDE4A KO $(n=$ 9), while batch 2 consisted of 5 mice each for WT and PDE4A KO (Figure 1). The data from 
the two batches were combined for statistical analysis. All behavioral tests were performed from 0900-1500 hours. The experimenter was blinded to the genotype of the animals, and animal testing order was randomized daily to reduce subjective bias. The battery was organized so that tests with minimal stress were performed first and tests with maximal stress and stimuli performed last (Figure 2.1). Before the testing paradigm began, animals were habituated (HAB) for 1 week by being transferred from the housing room to the testing room, being allowed to sit for $45 \mathrm{~min}$, gently handled, and then returned to the housing room. Testing for both batches started at 4 months of age and then progressively took place until 8 months of age following the protocol laid out in figure 1. All animals were housed in groups of 2-5 other than 1 WT mouse in batch 2, which was single housed (the behavioral data still fell within the group mean and was included in the analysis). Mice were allowed a minimum of $45 \mathrm{~min}$ to habituate to the testing room after transfer from the housing room before behavioral tests were initiated. All chambers and testing apparatuses used were cleaned with 70\% alcohol between animals to avoid any confounding effects of olfaction on behavior.

\section{Batch $1(n=9)$}

\begin{tabular}{|l|l|l|l|l|l|l|l|l|l|l|l|}
\hline HAB & OF & OR & EPM & HB & LDT & FST & TST & MWM & STPA & NSF* & EME \\
\hline
\end{tabular}

$\operatorname{Batch} 2(\mathrm{n}=5)$

\begin{tabular}{|l|l|l|l|l|l|l|l|l|l|}
\hline HAB & OF & OR & EPM & HB & LDT & FST & TST & STPA & NSF* \\
\hline \multicolumn{8}{|c|}{} \\
\hline
\end{tabular}

Figure 2.1. Behavioral Paradigm. Two batches of mice were used. Each batch consisted of two groups of PDE4A knockout (KO) and wild-type (WT) mice ( $n=9$ mice/group for batch 1 and $n=5 /$ group for batch 2$)$. The battery of behavioral tests was performed during days 1-34 in the following order: Habituation (HAB), open field (OF), object recognition (OR), elevated-plus-maze (EPM), hole-board (HB), light-dark-transition (LDT), forced-swim test (FST), tail-suspension test (TST), Morris Water Maze (MWM), step-through passive-avoidance (STPA), noveltysuppressed-feeding test (NSF), emesis test (EME). 


\section{Measurement of Locomotor Activity}

Mice were placed into individual PAS automated open field chambers (San Diego Instruments San Diego, CA). The number of light beam crossings for locomotor activity (horizontal crossings) and rears (vertical crossings) were recorded and averaged over six 5-min intervals (Hu et al. 2011).

\section{Anxiety-like behavior Tests}

Elevated plus-maze test. Mice were placed individually in the center of a plus-shaped maze (40 $\mathrm{cm}$ above the floor) facing an open arm. The number of entries (with all four paws in) and time spent in both open arms $(30 \times 5 \mathrm{~cm})$ and enclosed arms ( $30 \times 5 \times 15 \mathrm{~cm}$ high) were recorded for $5 \mathrm{~min}$. The level of anxiety-like behavior was determined by the percentage entries into open arms, and percentage time spent in open arms, which were calculated by: Open arm entry $(\%)=$ number of open arm entries/total number of arm entries x 100; Open arm time $(\%)=$ time $(\mathrm{s})$ spent in open arms/total time (s) in all arms x 100 (Li et al. 2009b).

Light-dark transition test. Mice were individually placed in the dark compartment $(15 \times 23 \mathrm{~cm})$ of a light-dark chamber. Anxiety-like behavior was determined by the following measures recorded for $5 \mathrm{~min}$ : the latency to cross through the hole $(8 \times 6 \mathrm{~cm})$ into the light compartment ( $30 \times 23 \mathrm{~cm}$, illuminated with a $60-\mathrm{W}$ bulb positioned $50 \mathrm{~cm}$ above), the time spent in the light compartment, and the number of transitions defined as the number of crossings from the dark compartment to the light side (Zhang et al. 2008).

Holeboard test. Mice were individually placed in the center of an open Plexiglas box ( $40 \times 40 \times 30$ $\mathrm{cm}$ ) with four holes ( $3 \mathrm{~cm}$ in diameter, $2 \mathrm{~cm}$ in depth) in the black floor and allowed to explore 
for $5 \mathrm{~min}$. Anxiety-like behavior was measured by recording the number of head-dips into the holes and the total time spent exploring the holes. Head dips were counted when the animal's head reached a depth sufficient enough to be even with its ears (Zhang et al. 2008).

Novelty suppressed feeding test. In brief, mice were deprived of food $24 \mathrm{~h}$ prior to the test. After $24 \mathrm{~h}$ of food deprivation, the mice were individually placed in the corner of a plastic open chamber $(35 \times 28 \times 16 \mathrm{~cm})$ covered by a $1-\mathrm{cm}$ thick layer of wooden bedding. A pellet of food (regular chow) was placed in the center of the floor, and a 60-watt bulb placed $50 \mathrm{~cm}$ above the center of the chamber. The latency to begin to chew the pellet, time spent digging, and the total amount of time spent eating were recorded for a total of $5 \mathrm{~min}$.

\section{Depression-like behavior Tests}

Forced-swim test. Mice were individually placed in a plastic cylinder $(45 \mathrm{~cm}$ high $\times 20 \mathrm{~cm}$ diameter) filled with water $\left(22-23^{\circ} \mathrm{C} ; 30 \mathrm{~cm}\right.$ in depth) for $6 \mathrm{~min}$ and the duration of immobility was recorded (Zhang et al. 2002). Immobility was defined as floating in an upright position without additional activity other than that necessary for the animal to keep its head above water. Fresh water was used for every other mouse.

Tail-suspension test. Mice were suspended from their tails and the duration of immobility (i.e., when the mouse was passively hanging and completely motionlessness) was recorded for a 6min period (Zhang et al. 2002). Mice were separated by black plastic partitions to avoid confounding effects of neighboring behavior. The effects of conspecific vocalizations however can't be ruled out.

\section{Memory Tests}


Object recognition test. This was carried out using the procedures described previously (Li et al. 2011). In brief, mice were trained over three 5 -min sessions separated by $24 \mathrm{~h}$ to discriminate a novel object from a familiar one in a plastic chamber $(40 \times 40 \times 20 \mathrm{~cm})$. In the first trial (T1), animals were placed in the chamber without an object and allowed to explore and habituate to the environment. Twenty-four hours later, animals were put back in the chamber for the training session (T2) and exposed to two identical objects (blue Lego blocks) located in two opposite corners of the box. The exploration time of each object was recorded to control for object and side bias. After $24 \mathrm{~h}$, mice were returned to the box to start the testing trial (T3), during which they were exposed to a familiar object (one of the Lego blocks from day 2) and a novel object (a red, cone-shaped, plastic glass), which was different from the familiar one in color and shape and placed in one of the same positions as the objects in T2. The exploration time for each object (Tf and Tn for familiar and novel objects, respectively) was recorded for determination of the recognition index $[R I=T n /(T n+T f)]$. Exploration was defined as facing or touching the object (within $2 \mathrm{~cm}$ of the object). The chamber and objects were thoroughly cleaned with $70 \%$ alcohol after every session to ensure olfactory cues did not play a role in the test.

Morris water-maze test. This test was used to assess spatial learning and memory following procedures described previously (Li et al. 2011) with slight modifications. In brief, mice were placed into a circular pool $(95 \mathrm{~cm}$ diameter $\times 25 \mathrm{~cm}$ high) in which a circular platform $(8.5 \mathrm{~cm}$ diameter $\times 15.5 \mathrm{~cm}$ high) was placed. The pool was then filled with water $\left(21 \pm 1^{\circ} \mathrm{C}\right)$ made opaque by the addition of powdered milk. Curtains were closed around the pool to eliminate any extraneous distal cues, and the water level was maintained so that the platform was $1 \mathrm{~cm}$ above the water. On the first day of the protocol, the visible cued-platform test was performed to 
control for sensory/motor abilities as well as motivation to exit the pool. A small red flag was placed on the platform and four visible cued-platform trials were run by placing the mouse at random starting locations, in which the animal was allowed to escape by finding the platform. The platform was placed at a new location for each trial. During the spatial acquisition trials started the next day, the platform was submerged $1 \mathrm{~cm}$ below the water surface and several extra-maze distal landmarks placed on the curtains. The mice were then trained for 15 trials over 3 days ( 6 trials day one, 5 trials day two, and 4 trials day three) to locate the hidden platform from different starting points (N, S, E, W, NW, and SE). Twenty-four hours after the last training session, a probe trial was conducted in which the platform was removed and the animals were allowed to swim for $60 \mathrm{~s}$. Assessment of spatial memory function was performed by analyzing swim speed, the number of entries, time spent and distance traveled in the target quadrant, and also the number of crossings over the circular area $(8.5 \mathrm{~cm}$ in diameter) where the hidden platform was previously located. Data was obtained using the EthoVision XT behavioral tracking system (Noldus, The Netherlands).

Step-through passive avoidance test. This was performed following procedures described previously (Li et al. 2011) with some modifications. In brief, the experiment was conducted using a two-compartment Plexiglas chamber (28 $\times 17 \times 25 \mathrm{~cm}$ high; ENV410B, Med Associates, St Albans, VT), which consisted of an illuminated and darkened compartment connected via a guillotine door and a metal grid floor connected to an isolated pulse stimulator. On the first day, mice were placed in the illuminated compartment of the chamber and allowed to explore both the illuminated and darkened compartments for 5 min. Twenty-four hours later during training on day 2, each mouse was placed in the illuminated compartment, and $60 \mathrm{~s}$ later the door was 
opened to the dark chamber. When the mouse entered the dark chamber, the guillotine door was closed behind it and the mouse administered a brief electric shock $(0.4 \mathrm{~mA} / 5 \mathrm{~s})$. Twenty seconds later the mouse was put back into the illuminated chamber, and the whole process was repeated until the animal reached the criterion of $60 \mathrm{~s}$ to enter the dark chamber after the door was opened, when the mouse was assumed to have learned the procedure and associated the shock stimulus with the dark side. The number of shocks before reaching the criterion was recorded to evaluate the learning ability of mice. On the testing day $24 \mathrm{~h}$ later, the mouse was once again placed in the illuminated compartment with the door closed for $60 \mathrm{~s}$, after which the door was opened, and the latency for the mouse to enter the darkened compartment was recorded for up to $300 \mathrm{~s}$. No shock was administered on the testing day for animals that entered the darkened compartment. The chamber was cleaned with $70 \%$ alcohol in between animals to avoid any confounding effects of olfaction on memory.

\section{Emetic-like behavior test.}

This was performed as described previously (Li et al. 2011) to examine the effects of PDE4A KO on emesis. In brief, animals were injected (i.p.) with xylazine $(10 \mathrm{mg} / \mathrm{kg}) / \mathrm{ketamine}(80 \mathrm{mg} / \mathrm{kg})$. Once the animals became ataxic, they were placed in the supine position in V-shaped metal troughs (covered by paper towels). The duration of anesthesia was determined as the time between the loss and return of the righting reflex; the latter was defined as when animals could right themselves three times within $30 \mathrm{~s}$ after being placed on their backs. 


\section{Determination of Corticosterone levels}

Urine samples were collected by picking the mouse up and holding it until the animal urinated on the plastic wrap. The urine was then pipetted into microcentrifuge tubes, and frozen at $-80^{\circ} \mathrm{C}$ until use. Following this procedure, the vast majority of mice urinated within $25 \mathrm{~s}$, which should have avoided any confounds of human interaction and immediate stress on corticosterone levels (Kurien and Scofield 1999). In addition, to control for diurnal variation of corticosterone, urine samples were collected at the same time (1200 h) every day over a course of 3 days during the novelty suppressed feeding test ( $24 \mathrm{~h}$ before food deprivation, during food deprivation, and 24 h after food deprivation). Corticosterone levels were determined using ELISA (ABNOVA, cat\# KA0468) as per manufactures instructions

\section{Microwave Fixation}

Animals were sacrificed by microwave fixation as described previously ( $O^{\prime}$ Callaghan and Sriram 2004). In brief, mice were transferred to NIOSH (Morgantown, WV) and allowed $3 \mathrm{~h}$ to habituate. Animals were then placed in a water-blanketed restrainer (TAW-174 applicator head) and immediately sacrificed in a 10 kW output Muromachi microwave applicator (Model TMW-4012C; Stoelting, Wooddale, IL) at an exposure level of $4.0 \mathrm{~kW}$ for $0.9 \mathrm{~s}$. The whole procedure normally took less than $15 \mathrm{~s}$ to complete.

\section{Tissue Preparation}

Microwaved tissues were prepared as described previously (O'Callaghan and Sriram 2004). In brief, microwaved brains were removed and brain specific regions dissected via coronal slices and the use of brain specific landmarks. Brain tissues were frozen at $-80^{\circ} \mathrm{C}$ until further use. 
Tissue lysate was prepared by sonicating in hot $\left(90^{\circ} \mathrm{C}\right) 1 \%$ SDS for $15 \mathrm{~s}$, and protein concentration quantified by standard BCA protein assay kit.

\section{Protein electrophoresis and Western blotting}

These were performed following procedures described previously (Li et al. 2011). In brief, $20 \mu g$ of hippocampal lysates were loaded onto Nupage 4-12\% bis-tris gels (Life Technologies WG1403BOX) and electrophoresis was performed at 120 v for $95 \mathrm{~min}$. All chemicals and buffers were made and used according to the Nupage Western blot protocol. After electrophoresis, samples were transferred to PVDF membranes at $75 \mathrm{v}$ on ice for $2 \mathrm{~h}$. Membranes were then blocked for $45 \mathrm{~min}$ at room temperature in Li-Cor blocking buffer (Li-Cor cat\# 927-4000), and incubated overnight at $4^{\circ} \mathrm{C}$ in a 1:1000 dilution of rabbit anti-PDE4A antibody (Abcam 14607) and 1:10,000 mouse anti-actin antibody (Abcam 8224) as a loading control. The next morning, membranes were washed $4 \times$ in PBST and Li-Cor secondary fluorescent antibodies goat-anti mouse IrDye $800 \mathrm{CW}$ and goat anti-rabbit 680RD were added in a 1:10,000 dilution for $40 \mathrm{~min}$ at room temperature to stain for actin and PDE4A, respectively. The membranes were washed 4 more times in PBST, and then analyzed using the Odyssey infrared imaging system to check for the presence of PDE4A and actin bands. 


\section{Statistical Analysis}

All data shown are means \pm SEM, and were analyzed using the GraphPad Prism 5 software (version 5.04). Urine ELISA corticosterone levels were plotted using 5-parametric analysis and MasterPlex ${ }^{\circledR}$ ReaderFit: Curve-Fitting Software for ELISA (Hitachi Solutions America, Ltd. MiraiBio Group) and then analyzed via repeated measures two-way ANOVA (2-ANOVA-R) with Bonferroni's post tests for multiple

comparisons; the rest of the

behavioral data were analyzed via

students two-tailed T-tests, or 2-way

ANOVA with repeated measures and

Bonferroni's post test for the

locomotor activity/rearing and

Morris Water Maze tests. Alpha

significance was achieved with a $\mathrm{P}$

value of $<0.05$

\subsection{Results}

\subsubsection{PDE4A $K O$ and locomotor}

activity. To determine the effect of

PDE4A KO on locomotor activity, animals were placed into a PAS-

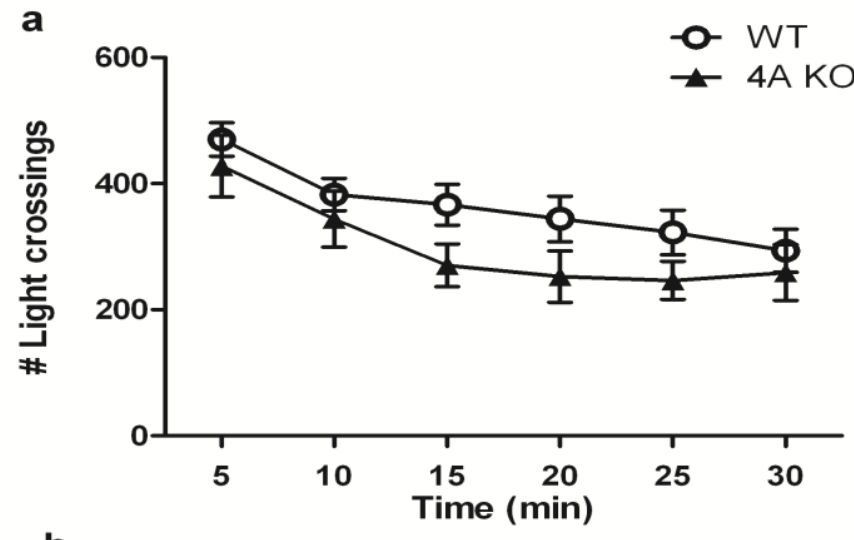

b

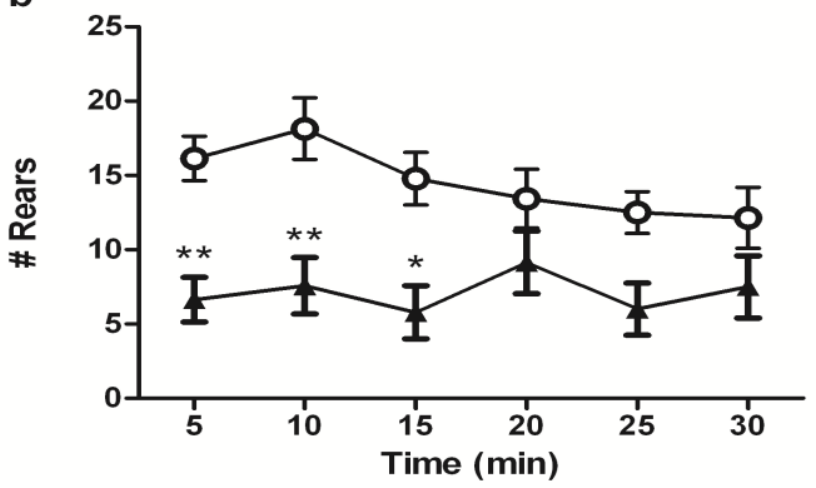

Figure 2.2. Effects of PDE4A KO on locomotor activity and rearing in the PAS Automated open field chamber. (a) PDE4A KO mice had no significant difference in locomotor activity compared to WT controls; however, they did have slightly lower activity in intervals 35. (b) PDE4A KO mice had significantly less rearing than WT mice, in particular during the first 3 intervals. Data shown represent means \pm SEM; $n=14$ /group; * $P<0.05$, ** $P<0.01$ vs. WT (two-way ANOVA with Bonferonni multiple comparison tests).

automated open-field chamber and the number of light crossings recorded and averaged over six 5-min intervals. There was no significant difference in locomotor activity between PDE4A KO 
mice and WT controls, although the KO mice did have a slightly lower baseline activity from 1520 min time (2-ANOVA-R: extremely significant effect of time, $F(5,130)=16.57, p<0.0001$; no effect of genotype, $F(1,26)=2.19, p=$ 0.151 ; no effect of genotype $\mathrm{x}$ time interaction, $F(5,130)=0.75, p=0.585$

Figure 2.2a). As an additional measure of exploratory activity, the number of rears was also recorded while the mice

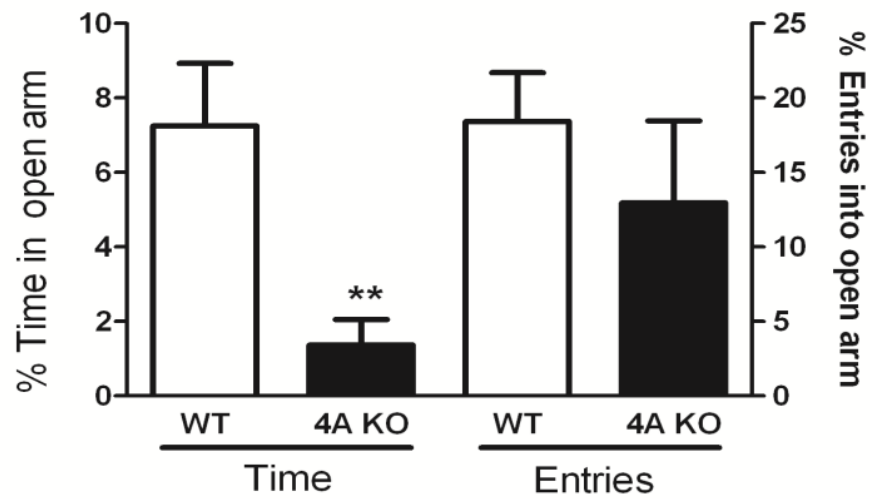

were in the open-field chambers. It was

found that PDE4A KO mice did show

Figure 2.3. PDE4A KO and the elevated plus maze test. PDE4A KO mice spent significantly less time in the open arms of the EPM relative to WT controls. They also had fewer entries into the open arm, although this was not significant

significantly lower rearing levels than WT

controls during the first $15 \mathrm{~min}$ of the test

(2-ANOVA-R: very significant effect of

PDE4A KO, $F(1,26)=12.77, p=0.0014 ;$

significant effect of time $x$ genotype interaction, $F(5,130)=2.36, p=0.0438$; no

effect of time, $F(5,130)=2.27, p=0.052$;

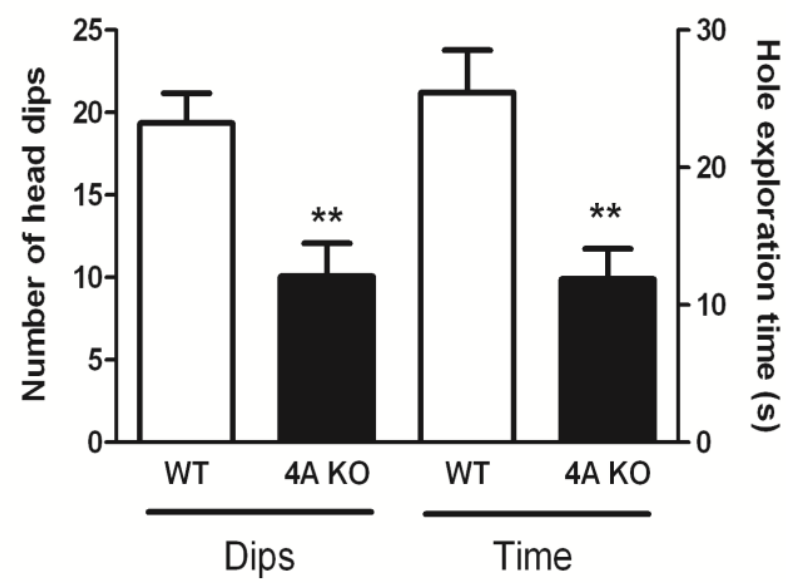

Figure $2.2 \mathrm{~b}$ ). This data indicates that while PDE4A KO did not induce lethargy as

Figure 2.4. PDE4A KO and the hole board test. PDE4A KO mice had significantly less head dips and hole exploration time in the HB test than WT controls.

interpreted by the lack of changes in horizontal light crossings, there was a decrease in vertical exploratory rearing which could be indicative of an anxiogenic-like phenotype. 


\subsubsection{PDE4A KO and anxiety-like behavior.}

To determine the effect of PDED4A KO on basal levels of anxiety, PDE4A KO and WT mice were tested in a variety of behavioral tests for anxiety-like behaviors, including elevated plus-maze (EPM, Figure 2.3), holeboard (HB, Figure 2.4), light-dark transition (LDT, Figure 2.5), and novelty suppressed feeding (NSF, Figure 2.6). In the EPM test, PDE4A KO mice spent significantly less time in the open arms of the maze when compared to WT controls ( $t=3.25, p=0.003$; Figure 2.3); they also had fewer entries into the open arms, although this was not significant $(t=$ 0.85, $p=0.40$; Figure 2.3). When tested in the HB test, PDE4A KO mice had significantly
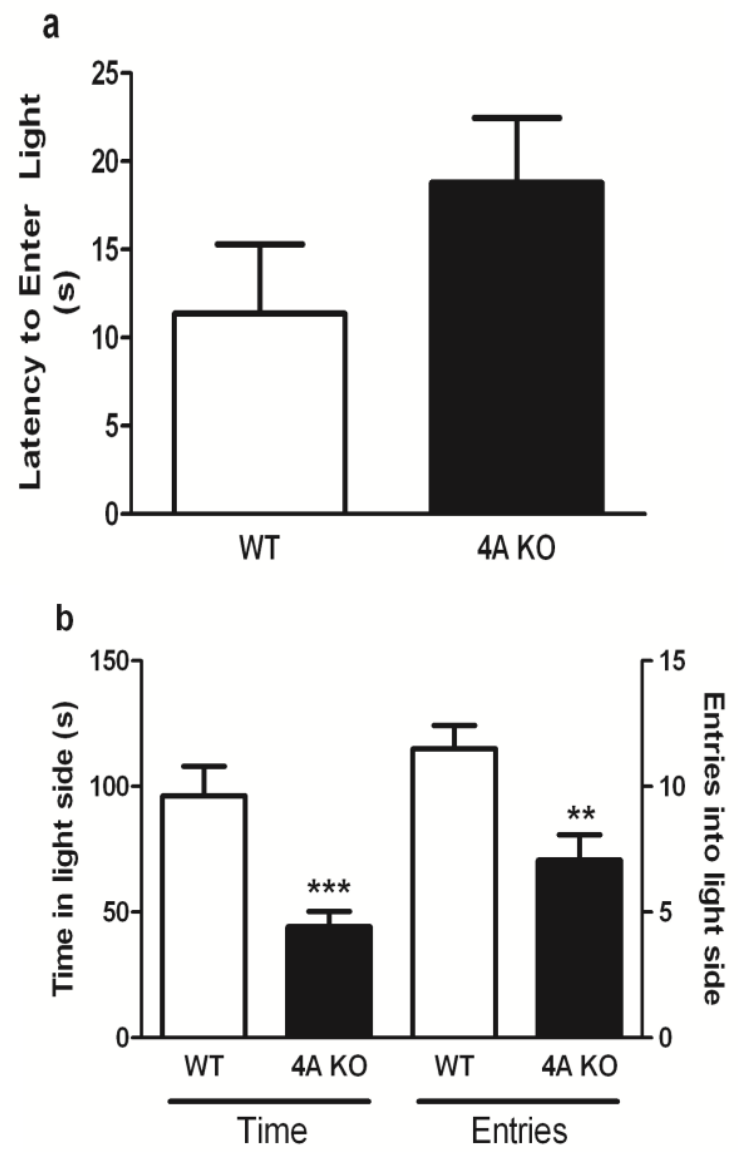

Figure 2.5. PDE4A KO and the light dark transition test. PDE4A KO mice had a longer latency to enter the light compartment, although the results were not significant. PDE4A KO mice also spent significantly less time in the light side, and had fewer entries into the light side relative to WT controls in the LDT test.

fewer exploratory head- dips $(t=3.43, p=0.0020$; Figure 2.4$)$ and also spent significantly less time $(t=3.60, p=0.001$; Figure 2.4) exploring (head-dipping) compared to WT controls. In the LDT test, PDE4A KO mice took longer to enter the light chamber but the results were not significant ( $t=1.375, p=0.1808$, Figure 2.5a), and PDE4A KO mice spent much less time in the light compartment $(t=3.94, p=0.0005$; Figure $2.5 b)$ and also had significantly fewer transitions into the light compartment $(t=3.23, p=0.003$; Figure 2.5$)$ than the WT controls. Lastly, in the 
NSF test after 24-h food deprivation, PDE4A KO mice had a much longer latency to eat the food

placed in the center of the novel, brightly lit

chamber, relative to WT controls $(t=3.15, p$

=0.004; Figure 2.6), and also spent a

significantly longer duration of the test

burying in the bedding than WT controls $(t=$

3.78, $p=0.002 ;$ Figure 2.6). These results

suggest anxiogenic-like behavior in PDE4A KO

mice.

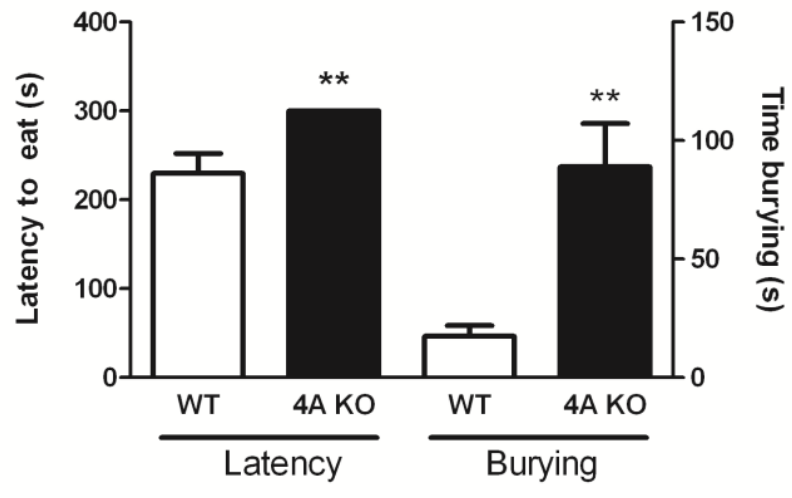

Figure 2.6. PDE4A KO and the novelty suppressed feeding test. PDE4A KO mice had a significantly longer latency to eat the chow, and also spent much more time burying than WT controls in the NSF test.

Urine corticosterone levels of PDE4A KO mice and WT controls were also analyzed

during the period of novelty

suppressed feeding. Urine was

chosen over plasma due to the

fact that urine levels are more

resistant to human handling

fluctuations which may confound

plasma levels of corticosterone

(Kurien and Scofield 1999). In

particular, we chose three time

points to observe the effect of

PDE4A KO on corticosterone

levels: (i) before stress (before

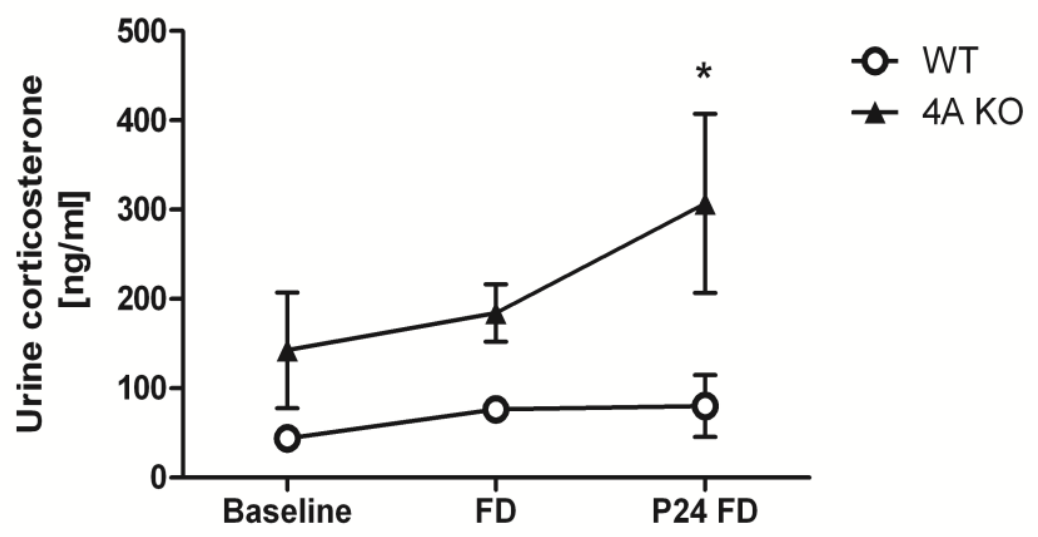

Figure 2.7. Effects of PDE4A KO on urine corticosterone levels. Urine samples were collected from PDE4A KO and WT mice to determine the levels of corticosterone $24 \mathrm{~h}$ before stress (baseline), during $24 \mathrm{~h}$ of stress induced by food deprivation (FD), and after stress had ended which was $24 \mathrm{~h}$ after food had been restored (P24 FD). PDE4A KO resulted in higher levels of corticosterone before and during stress although the changes were not significant; however, $24 \mathrm{~h}$ after the stress had ended, PDE4A KO mice had significantly higher corticosterone levels than WT mice. Data shown represent means \pm $\mathrm{SEM} ; \mathrm{n}=4$ /group; ${ }^{*} \mathrm{P}<0.05$ vs. WT ( 2 way ANOVA with Bonferonni multiple comparison tests). 
food was taken away), (ii) during stress ( $24 \mathrm{~h}$

of food deprivation), and (iii) after stress (24-h after food was restored). Interestingly, as expected, PDE4A KO mice did have slightly higher corticosterone levels before and during stress; however, they unexpectedly had significantly higher levels $24 \mathrm{~h}$ after the stress

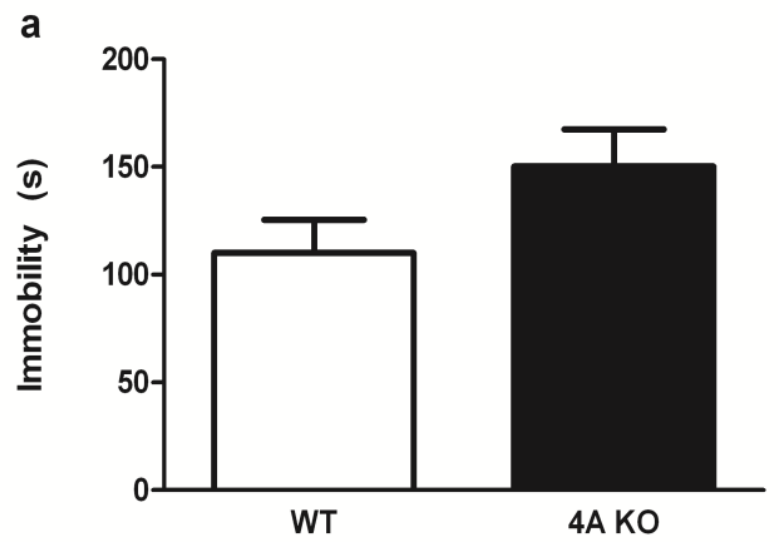
was restored (2-ANOVA-R: effect of genotype at P24 FD, $F(1,6)=4.99, p<0.05$; Figure 2.7). These data suggest that PDE4A KO mice may have some difficulty in terminating the activation of the HPA axis after exposure to a stressor when compared to WT controls.

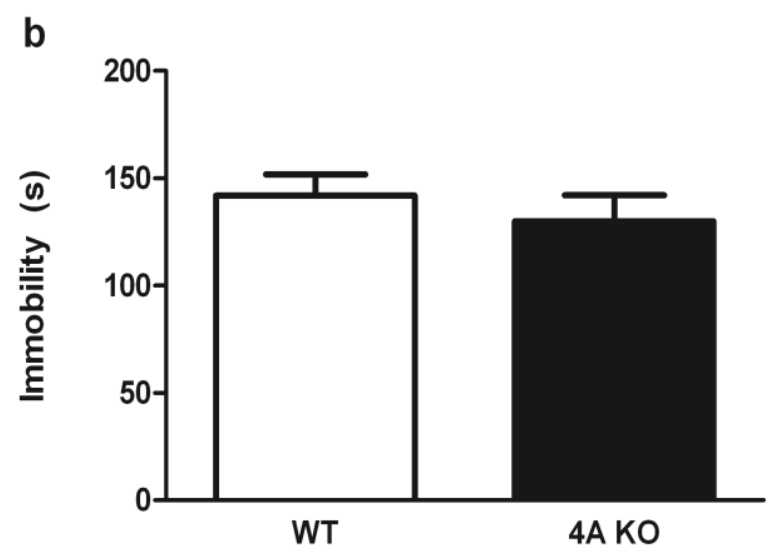

\subsubsection{PDE $4 A$ KO mice did not show significant changes in depressive-like behavior}

To identify the possible role that PDE4A plays in

Figure 2.8. Behavior of PDE4A KO and WT mice in the FST and TST. (a) PDE4A KO resulted in a slightly higher immobility time in the FST; however, the change was not significant. (b) PDE4A had no effect on immobility in the TST. Data shown represent means \pm SEM; $n=14$ /group (two-tailed Student's ttest).

depressive-like behavior, we tested PDE4A KO mice in the forced swim test (FST) and tail suspension test (TST). In the FST, PDE4A KO mice had slightly higher levels of immobility; however, this was not significant compared to the WT controls ( $t=1.74, p=0.093$; Figure 2.8a). In the TST, PDE4A KO had no effect on total immobility time ( $t=0.77, \mathrm{p}=0.449$; Figure $2.8 \mathrm{~b})$. These results indicate that PDE4A may not play a significant role in depressive-like behavior tested using the FST and TST. 


\subsubsection{PDE4A KO enhanced emotional memory but not spatial or episodic memory}

To determine whether PDE4A KO produces nootropic-like effects, PDE4A KO mice were tested in a variety of memory tests, including object recognition (OR), Morris water-maze (MWM), and step-through passive-avoidance (STPA) tests. In the OR test, PDE4A KO had no effect on novel object recognition on the testing day relative to WT controls $(t=0.50, p=0.625$;

Figure 2.9) suggesting that PDE4A may not be involved in mediating "episodic"-like memory.

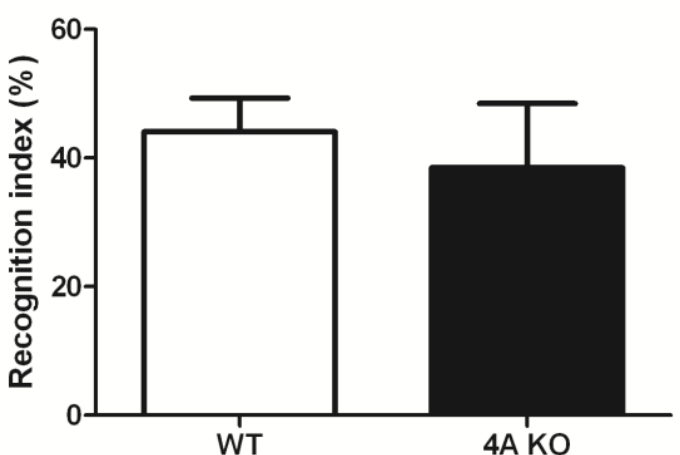

Figure 2.9. Effects of PDE4A KO on episodic memory in the object recognition test. PDE4A KO mice had no significant changes in the recognition index (RI) for the novel object on the testing day when compared to WT controls. Data shown represents the mean \pm SEM of $n=14$ /group (two-tailed Student's t-test).

To assess the effect of PDE4A KO on spatial memory, animals were trained in the MWM. Unexpectedly, PDE4A KO mice had significantly longer training latencies during trials 1 through 3 of the visible-platform test (2-ANOVA-R: significant effect of Time, $F(3,48)=12.76, p<0.0001$; significant effect of genotype, $F(1,16)=25.59, p=0.0001$; significant effect of genotype $x$ time interaction, $F(3,48)=5.58, p=0.002$; Figure 2.10a). During training with the invisible platform, PDE4A KO mice also had significantly longer latencies to reach the platform on days 1 and 3 when compared to WT controls (2-ANOVA-R: extremely significant effect of time, $F(2,32)$ $=13.84, p<0.0001$; very significant effect of genotype, $F(1,16)=9.00, p=0.009 ;$ no significant genotype $x$ time interaction, $F(2,32)=0.02, p=0.981$; Figure 2.10b). However, when tested during the probe trial, PDE4A KO mice performed similar to WT controls in regards to swim speed $(t=1.53, p=0.145 ;$ Figure 2.10c), distance traveled in the target quadrant $(t=1.07, p=$ 
0.303; Figure 2.10d), time spent in the target quadrant ( $t=0.84, p=0.415$; Figure 2.10e), and platform region crossings ( $t=0.53, p=0.604$; Figure $2.10 f)$. These results indicate that while PDE4A KO may produce some deficit in the learning phase of spatial memory, the retrieval
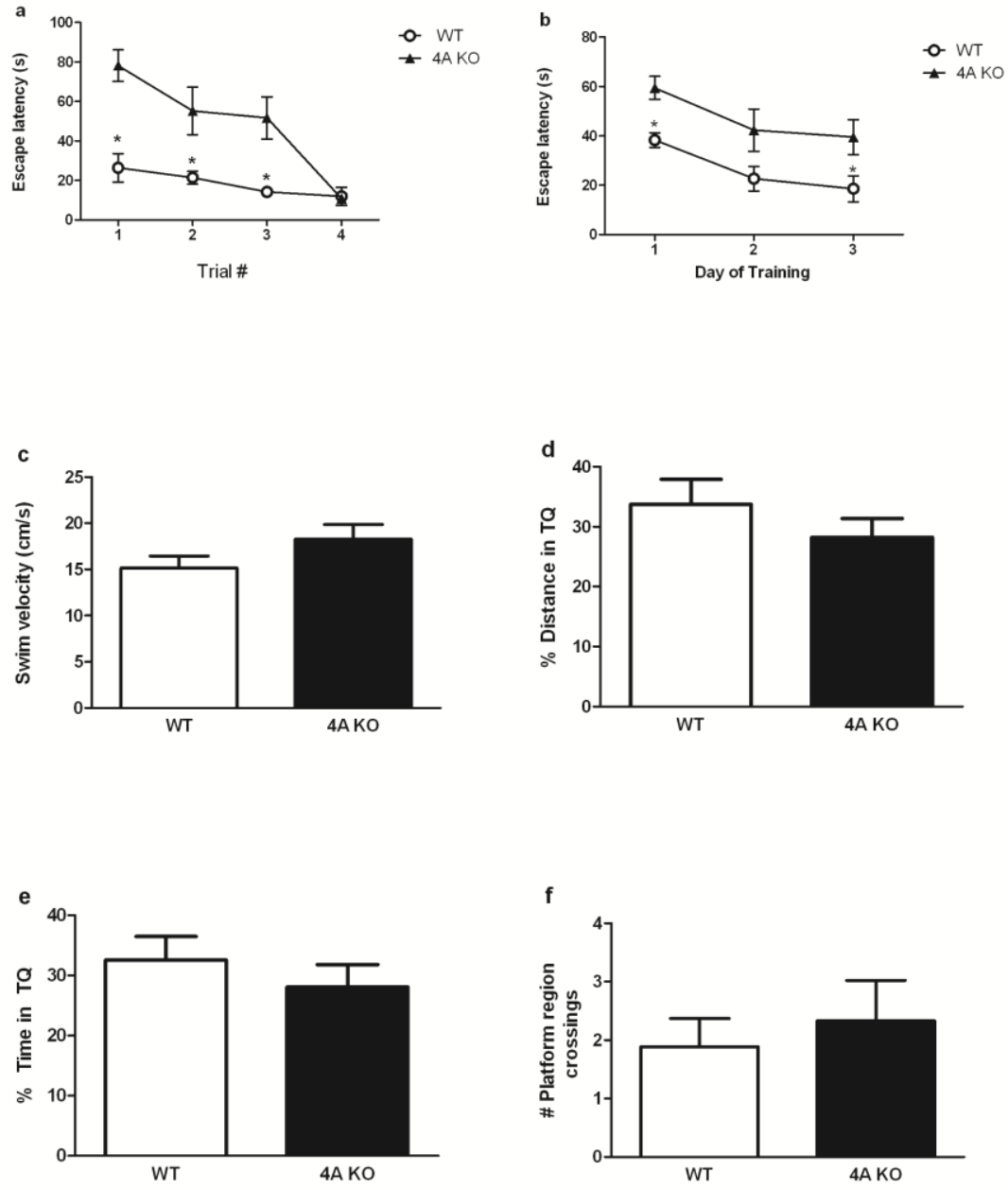

Figure 2.10. Effects of PDE4A KO on spatial memory in the Morris Water Maze (MWM) test. (a) In the cued visible platform test, PDE4A KO mice took significantly longer to find the visible platform during the first 3 trials compared to WT controls. (b) During the training of the hidden platform, PDE4A KO mice took significantly longer to find the hidden platform on each training day, although the results were only significant on days 1 and 3 . (c) PDE4A KO mice had no significant difference in swim speed measured during the probe trial test. (d-f) PDE4A KO mice had no significant difference in the \% total distance in the target quadrant (TQ) (d), \% time in the target quadrant (e), or the number of crossings over the previously located platform site (f) compared to WT controls. Data shown represent means \pm SEM; $n=9$ /group; * $\mathrm{P}<0.05$ vs. WT (two-way ANOVA with Bonferonni multiple comparison tests for $a$ and $b$, two-tailed Student's t-test for $c-f)$. 
phase as interpreted by the probe trial in the MWM test seems unaffected. To determine if deficits in vision were having a confounding effect on spatial memory training in PDE4A KO mice, we tested PDE4A KO mice and WT controls through electroretinograms (ERGs). PDE4A KO mice displayed no difference on the ERG in comparison to WT controls, suggesting they had no deficits in vision (Figure 2.11.).

Lastly, to determine the effect of PDE4A KO on "emotional" memory, animals were

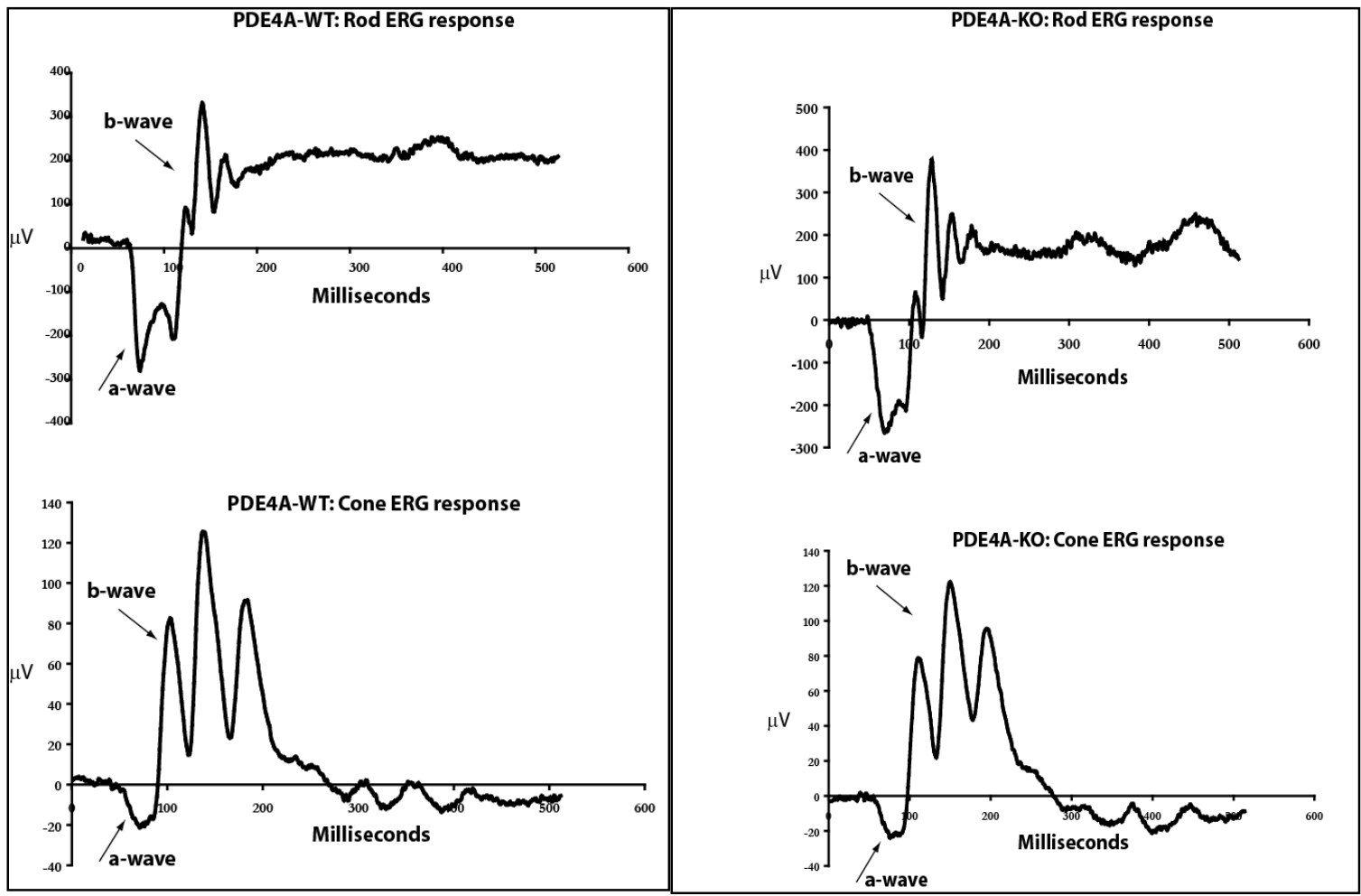

Figure 2.11. The effects of PDE4A KO on ERGs. To determine if PDE4A KO affected vision mice were subjected to ERGs. PDE4A KO mice displayed no change in a- or b- waves vs. WT controls, suggesting similar visual functions.

tested in the STPA test (Celikyurt et al. 2012). On the training day, PDE4A KO had no effect on the number of shocks required for the animal to "learn" the association between the dark 
chamber and the electric shock $(t=$

1.63, $\mathrm{p}=0.115 ;$ Figure 2.12a).

However, when tested $24 \mathrm{~h}$ later,

PDE4A KO animals had a significantly

longer latency to enter the dark

chamber than WT controls $(t=3.17, p=$

0.004; Figure 2.12b). This data suggests

that while PDE4A KO did not affect

episodic or spatial memory retrieval as

seen in OR and MWM, emotional

memory as measured via the STPA test

was significantly improved in mice

deficient in PDE4A.

To determine if PDE4A KO

affected nociception, which could have

had a confounding effect in the STPA

test, PDE4AKO animals and WT controls a

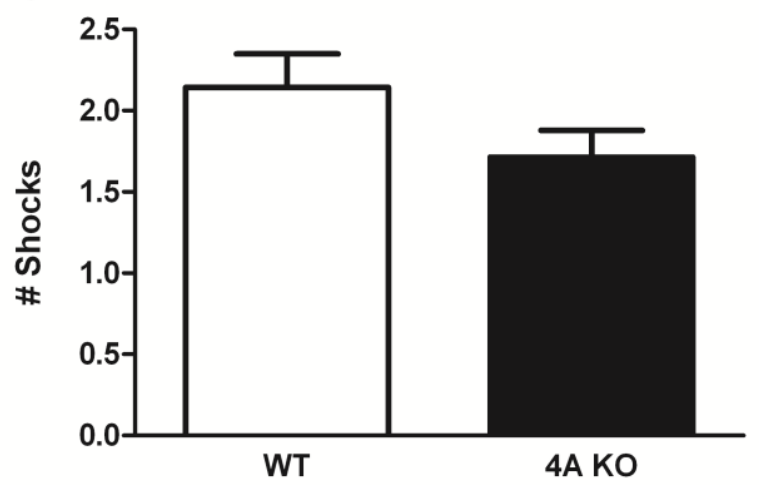

b

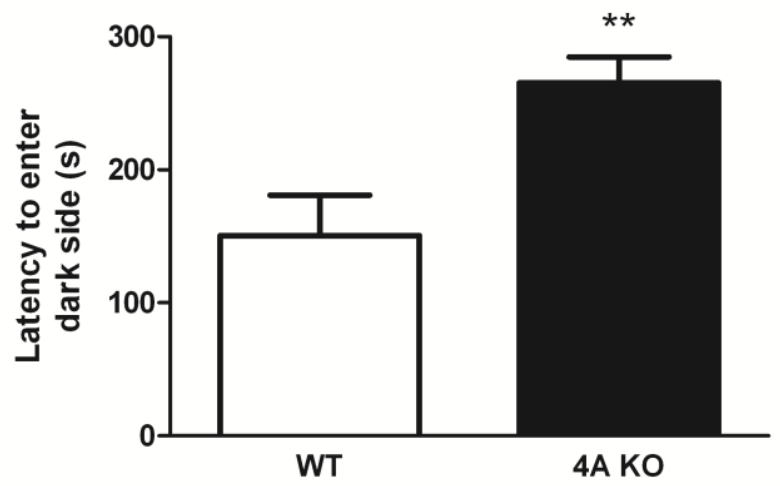

Figure 2.12. Effects of PDE4A KO on emotional memory in the step-through passive-avoidance test. (a) PDE4A KO mice had no significant changes compared to WT controls in the number of trials required to learn the association between the dark chamber and nociceptive shock (reaching the criterion of $60 \mathrm{~s}$ staying in the illuminated chamber). (b) PDE4A KO mice had a significantly longer latency to enter the dark chamber $24 \mathrm{~h}$ after training compared to WT controls. Data shown represent means $\pm \mathrm{SEM} ; \mathrm{n}=$ 14/group; ** P 0.01 vs. WT (two-tailed Student's t-test).

were tested on the hot plate. PDE4 KO mice displayed no changes in nociception compared to

WT controls (Figure 2.13).

2.3.5. PDE4A KO had no effect on emetic-like behavior. To examine the effects of PDE4A

$\mathrm{KO}$ on emesis, we also measured the xylazine/ketamine-induced duration of anesthesia in

WT/KO mice; this is an accepted emesis screen for rodents (Robichaud et al. 2002b; Burgin et 
al. 2010; Li et al. 2011). Typically, drugs

or treatments that induce emesis cause a

decrease in the duration of anesthesia.

We observed that PDE4A KO had no

effect on the duration of anesthesia (WT

$=89.00 \pm 6.49$ vs. $4 \mathrm{~A} \mathrm{KO}=95.25 \pm 6.95, \mathrm{t}$

$=0.66, p=0.521 ;$ Figure 2.14), although

rolipram-treated mice or PDE4D KO mice

showed decreased duration of

anesthesia (Li et al. 2011), suggesting

that PDE4A may not play a role in

emesis

\subsubsection{Loss of PDE4A expression in}

PDE4A KO mice

In addition to genotyping data (not

shown), Western blotting was

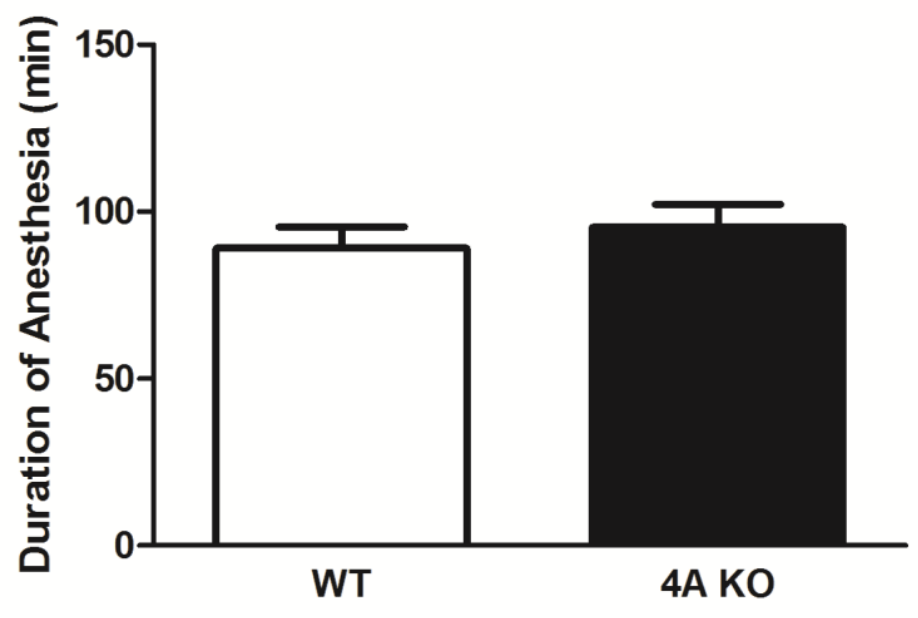

performed to confirm the

successful knockout of PDE4A in the

brains of PDE4A KO mice. Mice were

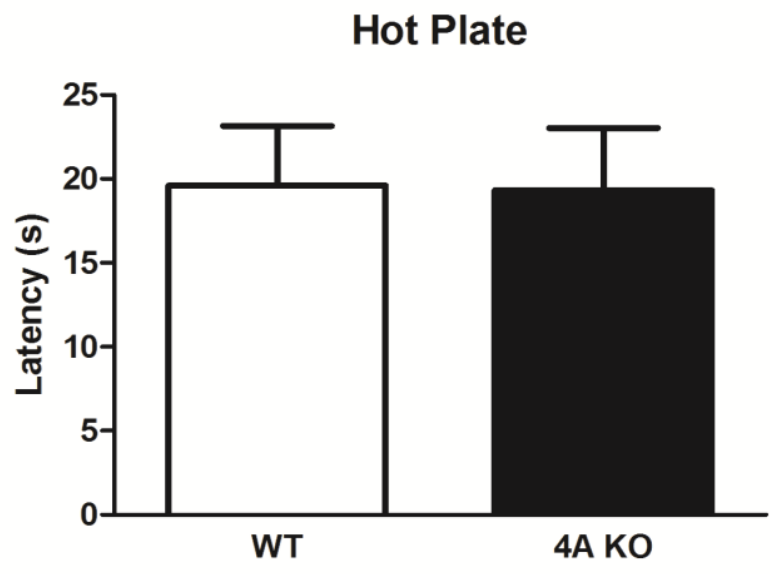

Figure 2.13. The effects of PDE4A KO on nociception. To determine if PDE4A KO affected nociception PDE4A KO and WT controls were tested on the hot plate. No difference in latency to withdraw the hindlimbs were displayed between the PDE4A KO and WT mice.

(5)

Figure 2.14. Emetic-like behavior test. PDE4A KO mice had no changed in the duration of ketamine/xylazine induced anesthesia, suggesting that PDE4A does not play a role in emesis.

sacrificed via microwave fixation, decapitated, and whole hippocampi dissected. Western

blotting analysis revealed that PDE4A KO mice had a complete deficiency of PDE4A5 and

PDE4A1 in the hippocampus as compared to WT controls (Figure 2.15). 


\subsubsection{PDE4A KO elevates CAMP}

and $p C R E B$ levels in the brain. To

determine if PDE4A KO affected

cAMP levels and downstream

signaling, cAMP ELISAs and PCREB

western blots were performed.

PDE4A KO mice displayed

elevations of cAMP in both the

cortex and hippocampus (Figure

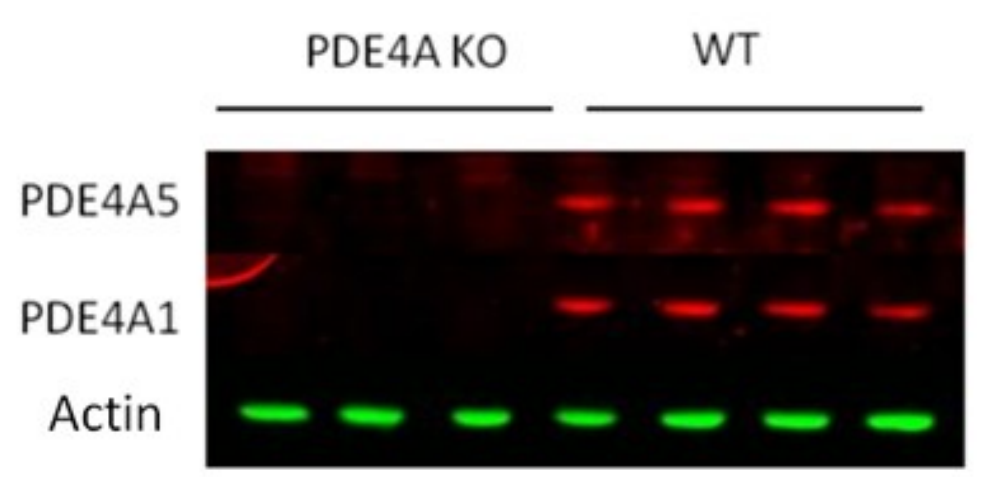

Figure 2.15. Confirmation of null PDE4A expression in PDE4A KO mice. The hippocampal tissues of PDE4A KO and WT mice were analyzed using Western blotting for the expression of PDE4A splice variants. PDE4A KO had a complete deficiency of PDE4A5 and PDE4A1 expression compared to WT controls, which had strong expression of both splice variants. This deficiency of PDE4As was not due to human errors as PDE4A KO still had strong, equal expression of $\beta$-actin compared to WT mice.

2.16). CREB and pCREB protein

levels were also investigated in the hippocampus and cortex of PDE4A KO mice via western blot

(Figure 2.17a). pCREB levels were significantly increased in the hippocampus of PDE4A KO, however this increase was not observed in the cerebral cortex (Figure 2.17b). In addition, to determine if this increase was due to a basal increase of CREB expression, CREB levels were also investigated. CREB levels were unchanged in the hippocampus (Figure 2.17c), suggesting the increase of PCREB was due to stimulation-induced activation.

\subsection{Discussion}

In this study we have shown through a battery of behavioral tests that PDE4A KO results in a strong anxiogenic profile and enhancement of emotional memory. PDE4A KO animals spent much less time in the open arms of the EPM, displayed decreases in both head dipping and exploration time in the HB test, had less transitions and time spent in the light chamber of the 
LDT, and had a much longer latency to eat food in addition to hyperactive digging in the NSF

test. A battery of anxiety tests was

used to ensure a true phenotype

was identified, as it has been

suggested running only one test

may not capture "trait" anxiety

(Ramos 2008; Steimer 2011). Thus,

running multiple anxiety tests

increases the chances of observing

true trait anxiety-like behavior as

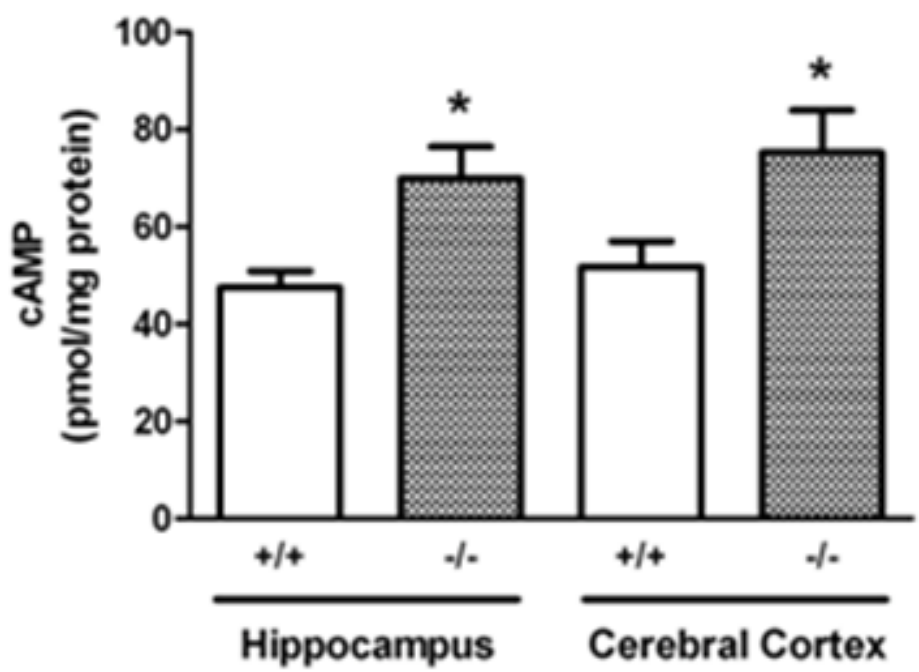

Figure 2.16. CAMP levels in PDE4A KO mice. cAMP levels were measured by ELISA in PDE4A KO and WT controls. PDE4A KO mice displayed elevated CAMP levels in both the cerebral cortex and the hippocampus.

opposed to a fluctuation of state anxiety which may be due to a single stressful event. In addition to measuring behavioral correlates of anxiety, we also measured urine corticosterone levels of PDE4A KO animals before, during, and after stress (before food deprivation, during food deprivation, and after food deprivation of the NSF test respectively). Urine corticosterone levels in PDE4A KO animals tended to be higher than WT before and during stress though the difference did not reach significance; however, urine corticosterone levels were significantly higher 24 hours after the stressor had been removed. This data suggests that PDE4A KO animals could have a deficiency involving negative regulation of the hypothalamic-pituitary axis (HPA), and could exhibit prolonged tonic release of corticosterone compared to WTs in response to a stressor.

In addition to the strong anxiogenic behavioral profile observed in PDE4A KO animals, we also observed an enhancement of "emotional" memory while other forms of memory seemed 
unaffected. In particular, PDE4A KO

animals had a significantly longer

latency to enter the dark chamber in

the testing day of STPA indicative of

an enhanced emotional memory

between the association of the dark

chamber and nociceptive electric

shock. It is important to keep in mind

that due to the fundamental

limitations of global PDE4A KO, we

were unable to determine whether

this memory enhancement was

through increased acquisition,

consolidation, or retrieval, thus

further research is needed on this

matter to elicit the specific

mechanisms involved. In contrast,
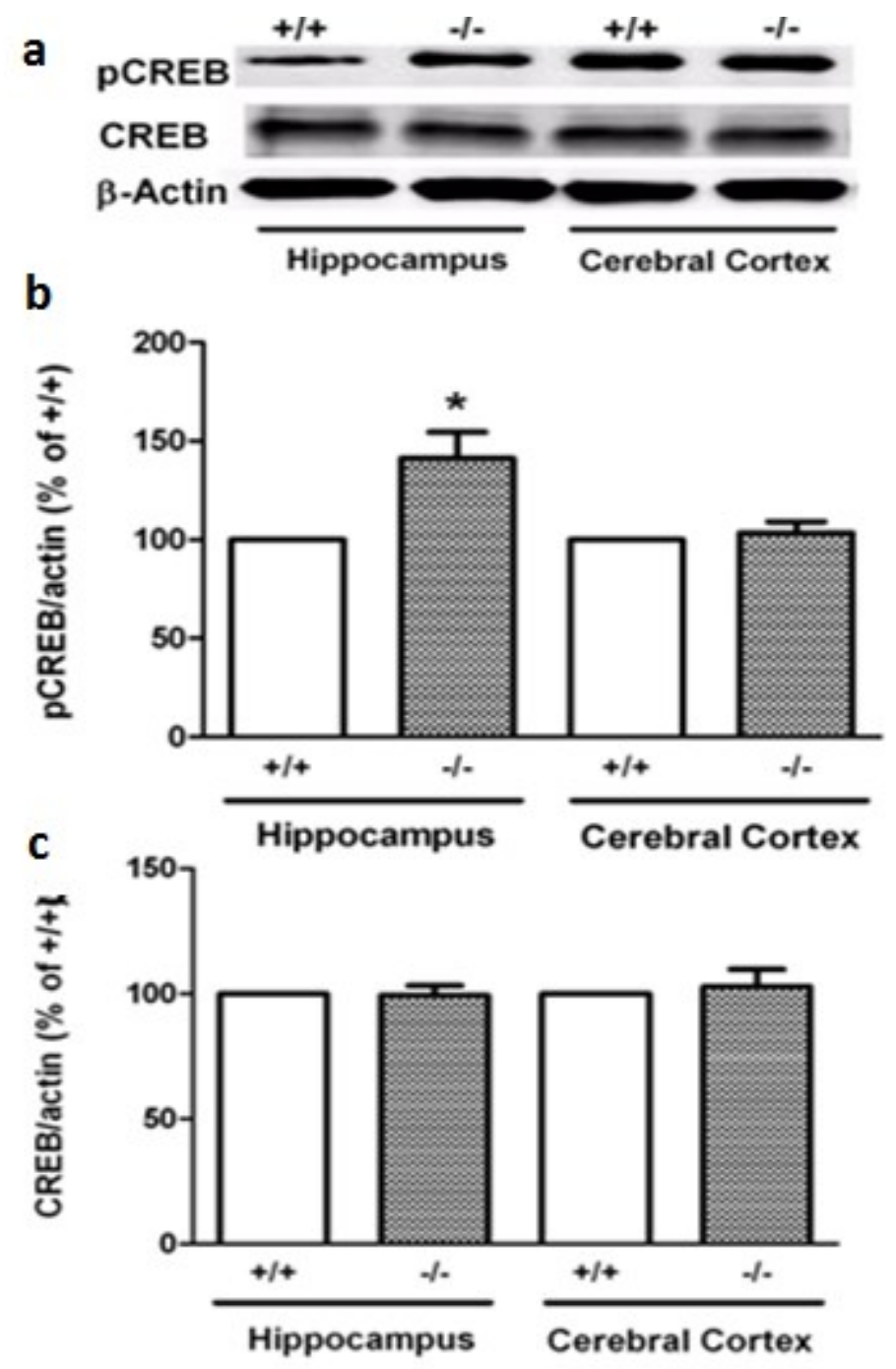

Figure 2.17. $\mathrm{pCREB} / \mathrm{CREB}$ protein levels in PDE4A KO mice. CREB/pCREB levels were measured by western blot in PDE4A $K O$ and WT controls. PDE4A KO mice displayed elevated pCREB levels in the hippocampus; CREB levels were unchanged.

when tested in the OR test which is representative of episodic memory, PDE4A KO animals showed no improvement over WT controls. Interestingly, when testing spatial memory in the MWM, PDE4A KO animals had significantly longer latencies on training days 1 and 3 to find the hidden platform, but performed similarly to controls during the probe test day. This data suggests that while the acquisition or consolidation of spatial memory may be impaired in 
PDE4A KOs, the retrieval phase seemed unaffected. In addition, PDE4A KOs also had significantly longer times to reach the platform during the first 3 trials of the cued visible platform test. While this data may represent a deficit in motivation to escape, it is more likely that PDE4A KOs had deficits in other areas such as visual-spatial working memory, or visual acuity since the latency for PDE4A KOs to reach the visible platform did improve during each trial (suggesting that reaching it was a rewarding experience and they were motivated to escape faster). Also, PDE4A KOs had no difference in swim velocity compared to WTs suggesting physical limitations were not the reason for the increased latency to reach the platform. One explanation for the attenuation in the training phase and visible platform test of the MWM could be due to elevated levels of corticosterone and anxiety-like behavior induced by PDE4A KO as it has been shown that both anxiety and elevated glucocorticoids can interfere with spatial memory learning (Korte 2001; Vasconcellos et al. 2003; Shackman et al. 2006). PDE4A KO animals showed no significant changes in behavioral depression tests, though PDE4A KOs did tend to have higher immobility in the FST. The behavioral effects of PDE4A KO on anxiety and emotional memory appear unrelated to sedation and lethargy as PDE4A KO appeared to have no significant effects on locomotor ability.

While this study has suggested that PDE4A is involved with the mediation of anxiety like behavior and emotional memory, the mechanisms through which it does so have yet to be identified; however, there are many possibilities for how PDE4A KO could lead to anxiogenesis. Indeed it has been shown that PDE4A is strongly expressed in brain regions important for anxiety such as the prelimbic cortex, anterior cingulate cortex, piriform cortex, hippocampus, and amygdala (McPhee et al. 2001). When considering the mechanisms through which PDE4A 
might mediate anxiety, alterations of cAMP levels should be at the forefront of the discussion due to cAMP-hydrolysis being the defining characteristic of PDE4A function (Conti et al. 2003b; Houslay and Adams 2003; Zhang 2009). Theoretically, PDE4A KO should lead to an increase of cAMP levels in the aforementioned brain regions. In agreement, it has been shown previously that PDE4B KO, which also results in anxiogenic like behavior, leads to increased cAMP levels in the various brain regions in which it is expressed (Zhang et al. 2008). Increasing cAMP levels in anxiety-related brain regions such as the medial pre-frontal cortex (mPFC), amygdala, and hippocampus could have a significant impact on the manifestation of anxiety like behavior and activation of the HPA axis depending on the region of interest. To better understand how elevated cAMP levels could change anxiety like behavior, it is first important to understand the individual roles that these brain regions play in the stress response.

The control of anxiety-like behavior is greatly mediated by a balance of mPFC and amygdala activity in a top down vs. bottom up struggle of dominance (Hains and Arnsten 2008; Arnsten 2009; Gamo and Arnsten 2011). It has been shown that this control is tightly regulated, and the presumed increases in CAMP that should accompany PDE4A KO could have significant changes on the functional and anatomical characteristics of these areas. Generally, it is believed that under normal circumstances the MPFC plays an inhibitory role on the stress response (Hains and Arnsten 2008; Arnsten 2009). This is thought to be through glutamatergic projections of the MPFC to the anterior bed nucleus of the stria terminalis (aBNST), which then sends inhibitory GABAergic projections to the periventricular nucleus (PVN) of the hypothalamus (Radley et al. 2009; Radley and Sawchenko 2011). The PVN of the hypothalamus is the main initiator of the HPA axis through the release of corticotrophin releasing factor (CRF) 
(Radley and Sawchenko 2011) which then stimulates release of adrenocorticotropic hormone (ACTH) from the anterior pituitary. Once ACTH is in the blood stream, it can then stimulate release of corticosterone from the adrenal glands (Radley et al. 2008) which in turn mediates many of the effects of the stress response through mineralcorticoid (MR) and glucocorticoid (GR) receptor activation (Korte 2001). Indeed, it has been shown that lesions to the mPFC can result in elevated corticosterone probably due to reduced inhibitory tone on the HPA axis (Brake et al. 2000; Radley et al. 2006a). In addition, it has also been shown that the mPFC projects to the amygdala, and that lesions to the mPFC can result in increased c-fos (putative marker for neuronal activity) expression in the medial amygdala indicative of hyperactivity (Figueiredo et al. 2003).

While it is believed the mPFC plays an inhibitory role in the stress response, in actuality this may be an oversimplification, as there are many conflicting reports on the effects of mPFC lesions or inactivation. Groups have shown that lesions or inactivation of the MPFC can result in attenuations of the stress response (less anxiety, decreased HPA activity) (Gonzalez et al. 2000; Deacon et al. 2003; Shah and Treit 2003; Shah and Treit 2004; Shah et al. 2004; SierraMercado et al. 2006), increases in the stress response (Figueiredo et al. 2003), or no effects on anxiety (Lacroix et al. 1998). While these results may at first seem difficult to interpret, it is very possible that these contradictory findings may be due to inconsistencies between the lesions of different groups targeting the entire MPFC as opposed to specific mPFC sub-regions. Many groups have used lesions encompassing the entire mPFC, and thus have treated it as one unitary homogenous region (Shah and Treit 2003); however, a more recent and accurate approach suggests that the MPFC may actually have functional segregation along its dorsal- 
ventral sub-regions which have contrasting roles in the mediation of the stress response (Holmes and Wellman 2009). Radley and colleagues have provided compelling evidence for the segregation of mPFC sub-regions in the regulation of the stress response (Radley et al. 2006a). They have shown that lesions to the dorsal prelimbic (PL) mPFC result in an increase of c-fos protein and CRF mRNA expression in the PVN after restraint stress compared to sham controls. However, lesions to the ventral infralimbic (IL) mPFC had the exact opposite effect and resulted in a decrease of c-fos protein and CRF mRNA in the PVN. In addition, the dorsal PL lesioned group had significantly higher corticosterone levels than sham controls after restraint, while the ventral IL lesioned group had lower corticosterone levels than sham post stress (Radley et al. 2006a). These results suggest that the dorsal PL-mPFC may play an inhibitory role, while the ventral IL-mPFC may play an excitatory role on the HPA axis and stress response. Other groups have made similar findings, as it has been shown that inhibiting synapses in the PL-mPFC during restraint stress significantly increased the tachycardiac response, while inhibiting synapses in the IL-mPFC reduced the tachycardiac response to stress (Tavares et al. 2009). These results are not surprising, as it has been shown that the prelimbic and infralimbic regions have significantly different connections in the brain and functional characteristics (Heidbreder and Groenewegen 2003; Vertes 2004). It is interesting to note that PDE4A may be expressed differently in the PL-mPFC vs the IL-mPFC (McPhee et al. 2001), identifying these brain regions as a possible site of future investigation in regards to the mediation of anxiety by PDE4A.

During times of relaxation and normal wakefulness, the MPFC is tonically active and the amygdala, which is one of the defining anxiety and negative emotion related brain areas, is more hypoactive (Arnsten 2009). However, when a stressor occurs, the amygdala is activated, 
which in turn activates catecholaminergic areas in the brain stem such as the locus coeruleus (LC) and ventral tegmental area (VTA), which are responsible for noradrenergic and dopaminergic projections to the brain, respectively. Upon stress, axonal projections from the LC and VTA that terminate in the MPFC release a surge of norepinephrine (NE) and dopamine (DA) which have significant effects on the activity of MPFC pyramidal neurons (Hains and Arnsten 2008; Arnsten 2009; Arnsten et al. 2010; Gamo and Arnsten 2011).

As previously mentioned, the dorsal PL-mPFC has been suggested to play an inhibitory role in the mediation of anxiety-like behavior and HPA axis activity. However, the level of inhibition from the PL-mPFC onto the HPA axis is related to the pyramidal-neuron activity within the PL area, and this activity has been shown to be tightly regulated. The Arnsten lab has been instrumental in characterizing the functional regulation of pyramidal dendritic spine activity in the MPFC, and has termed this "dynamic network connectivity" as a new form of neuroplasticity (Arnsten et al. 2010). Under normal wakeful conditions pyramidal dendritic spine activity in the MPFC is under a fine balance of excitatory control and inhibitory "sculpting" via $\alpha 2 a$ adrenergic receptors and dopamine D1 receptors respectively, both of which can mediate cAMP levels in the neuron (Arnsten 2009; Arnsten et al. 2010; Arnsten 2011). In particular, it has been shown that cAMP levels have significant control over pyramidal activity in the PL-mPFC area via the activation of hyperpolarization-activated cyclic nucleotide-gated ( $\mathrm{HCN}$ ) channels. Upon activation by CAMP, $\mathrm{HCN}$ channels open and allow the efflux of $\mathrm{K}^{+}$ions out of the cell, resulting in hyperpolarization, and subsequent decreased excitability of the dendritic spines in which they are located. Normally, cAMP levels of the PL-mPFC are kept in check by tonic low level activation of the $\alpha 2 \mathrm{~A}$ receptors, which are the high affinity NE 
receptors and $\mathrm{G}_{\alpha \mathrm{i}}$ coupled to suppress cAMP production. This suppression of cAMP levels maintains the low-level activation of the PFC neurons to maintain a working memory. In conjunction with the low levels of NE, normal low levels of dopamine also "sculpt" the neurons and subsequent working memory by binding to D1 receptors which are $\mathrm{G}_{\text {as }}$ coupled and increase CAMP production. This elevation of cAMP production "sculpts out" the non-specific neurons not being used in the maintenance of a working memory by turning them off through activation of HCN channels. However, during stressful conditions, the high levels of DA and NE that are released into the PL significantly increase the activity of HCN channels and reduce the firing of all PL-mPFC pyramidal neurons (Ramos et al. 2006; Arnsten et al. 2010; Arnsten 2011; Gamo and Arnsten 2011; Arnsten and Jin 2012). This is in party by an exaggerated activation of D1 receptors, as well as binding of NE to lower affinity $\alpha 1$-adrenergic receptors which activate the PKC pathway and subsequently produce CAMP in a $\mathrm{Ca}+$ dependent manner via $\mathrm{Ca}+$ sensitive adenylyl cyclases (Hains et al. 2009). Activation of $\alpha 2 A$ receptors by Guanfacine (a selective $\alpha 2 \mathrm{~A}$ adrenergic agonist) has been shown to reduce cAMP signaling and $\mathrm{HCN}$ activity, and increase firing of PR pyramidal neurons (Ramos et al. 2006; Wang et al. 2007b). Additional support for the importance of the LC and noradrenergic innervation in the PL-mPFC has been shown by Radley and colleagues (Radley et al. 2008). They have shown that selective ablation of LC-projected noradrenergic terminals in the PL-mPFC during stress reduced the amount of cFOS and CRF expression in the PVN (Radley et al. 2008). This system in the PL-mPFC is tightly controlled, and PDE4A could be a key regulator. PDE4A KO should theoretically elevate cAMP levels in the MPFC, activating HCN channels and reducing PL-mPFC pyramidal firing. This decrease in PL-MPFC activity should result in suppressed inhibition of the PVN and the HPA axis, 
and could account for the elevated corticosterone and anxiogenesis observed in PDE4A KO animals. Thus, PDE4A may be an important regulator of the activity of the PL-mPFC through dynamic connectivity and modulation of the stress response, and could explain the strong anxiogenic phenotype observed in PDE4A KO animals.

While it has been shown that stress and elevations in cAMP may make the MPFC weaker, it has the exact opposite effects in the amygdala. After acute stress the mPFC shows reduced functional activation, and after chronic stress reduced dendritic branching and length (Radley et al. 2004; Radley et al. 2006b). Interestingly, stress results in the strengthening of the amygdala and increased spinogensis (Vyas et al. 2002; Lakshminarasimhan and Chattarji 2012). One possible mechanism for this increased spinogensis may be through cAMP and downstream CREB signaling. Once CAMP is elevated in a neuron, it can then activate PKA, which relocates to the nucleus and phosphorylates CREB (pCREB) (Finkbeiner et al. 1997; Shaywitz and Greenberg 1999; Carlezon et al. 2005; Hansen and Zhang 2013). CREB is a transcription factor which binds CAMP response elements (CRE sites) within promoter regions of genes enhancing transcription of the specific product (Carlezon et al. 2005; Alberini 2009). One common downstream target of $c A M P / p C R E B$ signaling is BDNF, which has been extensively shown to increase dendritic spinogensis (Lakshminarasimhan and Chattarji 2012; Kaneko et al. 2012; Vigers et al. 2012; Hiester et al. 2013; Orefice et al. 2013). Interestingly, it has been found that after stress both pCREB (Adamec et al. 2011) and BDNF (Lakshminarasimhan and Chattarji 2012) levels are elevated in the amygdala. Since PDE4A is strongly expressed in the amygdala (McPhee et al. 2001), PDE4A KO could elevate cAMP levels in the amygdala resulting in a subsequent increase of $\mathrm{pCREB}$ and BDNF-mediated spinogensis. This proposed cAMP/CREB mediated strengthening 
of the amygdala could then result in hyperactivation of the amygdala and additional inhibition of the PL-mPFC, possibly through LC noradrenergic projections as previously mentioned. Indeed, it has been shown that amygdala activation can decrease PL-mPFC activity (Garcia et al. 1999). In addition, it has been shown that over-expression of CREB in the amygdala increased anxiety-like behavior in the EPM test (Wallace et al. 2004). Thus, the observed anxiogenic phenotype and increased corticosterone observed in PDE4A KO animals may have a combined effect of PL-mPFC hypoactivation, and amygdala hyperactivation.

The enhanced emotional memory of PDE4A KOs observed in the step-through passiveavoidance test may also be related to amygdala-based mechanisms and the subsequent increased anxiety and corticosterone levels seen in PDE4A KO mice. Indeed it has been shown that the amygdala plays a strong role in emotional memory (McGaugh 2004), and elevated corticosterone causes increased amygdala-based emotional memory consolidation via stimulation of GRs (Korte 2001; Roozendaal et al. 2009). In addition, it has also been shown that heightened trait anxiety can lead to nootropic effects on amygdala-based fear and emotional memory paradigms (Yen et al. 2012). Thus it may be possible that the increased anxiety and corticosterone caused by PDE4A KO and amygdala hyperactivity could lead to enhanced emotional memory. Elevated cAMP levels caused by PDE4A KO would almost certainly lead to an increase of PKA activation, which has also been shown to significantly promote the consolidation of emotional-based memory in the amygdala (Arnsten et al. 2005). Interestingly, this could also explain why there were no improvements in the MWM, as spatial memory has been shown to be more directly correlated to dorsal hippocampal function, as well as to be negatively affected by anxiety and corticosterone (Korte 2001; Vasconcellos et al. 2003; 
Shackman et al. 2006). In addition, we saw no effect of PDE4A KO on the effects of OR memory which is representative of episodic like memory (Ennaceur 2010). While the hippocampus is important for contextual object recognition, it is not as important for non-contextual object recognition where the objects stay in the same place and only the characteristics of the novel object change. This paradigm has actually been shown to involve more of the perirhinal cortex than the hippocampus (Winters et al. 2008). While PDE4A is strongly expressed in the hippocampus, it has not yet identified as having strong expression patterns in the perirhinal cortex and thus may explain why OR memory was unchanged (McPhee et al. 2001). It would be interesting to see if PDE4A KO would have an effect on contextual novel object recognition in which the locations of the objects are changed, and thus more of the hippocampus is used. Interestingly, one study has indeed shown that PDE4A is involved with memory deprivation following sleep deprivation (Vecsey et al. 2009)

The hippocampus has also been shown to play an important role in anxiety like behavior. In particular, the ventral hippocampus (VHPC) has been shown to play a more significant role in the regulation of anxiety-like behavior than the dorsal hippocampus (Bannerman et al. 1999; Bannerman et al. 2003; Degroot and Treit 2004; Adhikari et al. 2010). PDE4A is strongly expressed in the hippocampus (Cherry and Davis 1999; McPhee et al. 2001); however, there has yet to be a study comparing PDE4A expression levels between the dorsal and ventral axis. The role of the ventral hippocampus in anxiety is controversial, as studies have shown it to play both an excitatory (Bannerman et al. 2003; McHugh et al. 2004) and inhibitory (Radley and Sawchenko 2011) role in the stress response suggesting that there may be additional segregation within the ventral hippocampus. It is possible that increased cAMP 
signaling due to PDE4AKO could strengthen the ventral hippocampus due to increased pCREB and BDNF mediated spinogensis, though this is less clear than other brain regions. Theta rhythm in the VHPC could play a significant role as well in the expression of anxiety-like behavior. Interestingly, it has been shown that theta-rhythm in the vHPC correlates with anxiety like behavior (Adhikari et al. 2010; Adhikari et al. 2011) and that these theta rhythms are increased in 5- $\mathrm{HT}_{1 \mathrm{~A}} \mathrm{KO}$ mice (Gordon et al. 2005) which have increased anxiety (Heisler et al. 1998). The $5-\mathrm{HT}_{1 \mathrm{~A}}$ receptor is $\mathrm{G}_{i}$ coupled, thus these mice could have elevations of cAMP just like PDE4A KO mice could. It is possible that PDE4A KO elevated cAMP levels in the ventral hippocampus, which resulted in increased theta rhythm and anxiogenic behavior.

There is much additional evidence suggesting cAMP levels and downstream signaling could have significant effects on anxiety like behavior and the activation of the HPA axis and stress response. Interestingly, it has been shown that the anxiogenic corticotropin-releasing factor (CRF) increases CAMP signaling, and the anxiolytic neuropeptide $\mathrm{Y}$ decreases CAMP signaling in an immortalized AR-5 amygdala cell line (Mulchahey et al. 1999; Sheriff et al. 2001). In addition, it has also been shown that elevations of BDNF (which could result from increased cAMP/pCREB inPDE4A KO) in the PVN increase CRF release, and subsequent activation of the HPA axis (Jeanneteau et al. 2012). These studies complement our PDE4A KO behavioral data and theory that increased cAMP signaling could cause anxiety-like behavior. In particular, corticosterone levels in PDE4A KO animals tended to be higher before and during stress (though the difference was not significant), however they were significantly higher 24 hours after the stressor had been removed. Since PDE4A is the "off switch" to cAMP signaling, it is possible that after the stressor had been removed cAMP levels remained elevated and persisted much 
longer in PDE4A KO animals than WT animals. This loss of cAMP hydrolysis could have caused the extended release of CRF and subsequent increase of corticosterone we observed 24 hours post stress in the PDE4A KOs.

At this point these theories are purely speculative and additional research is needed to confirm our hypotheses. While we have suggested that increased cAMP may be responsible for anxiety like behavior, it is important not to oversimplify this and generalize this to every area of the brain. For example, it has been shown that PDE4D KO has antidepressant and nootropic like properties (Zhang et al. 2002; Li et al. 2011), contrasting the anxiogenic effects observed in PDE4B KO (Zhang et al. 2008) and PDE4A KO. While the effects of increased cAMP and pCREB in the hippocampus are nootropic and antidepressant, increasing PCREB in the nucleus accumbens promotes pro-depressive like behavior and anhedonia (Muschamp et al. 2011). In addition to brain region-specific expression of PDE4 subtypes, the complexity is further increased by splice variant expression and regions with overlapping expression patterns. The different PDE4 subtypes and splice variants may even be having different effects within the same neuron as compartmentalized micro-domain signaling has been shown to be extremely important within cAMP signaling pathways (Houslay and Adams 2003; Conti et al. 2003a; Houslay 2010). These studies point to the incredible complexity when dealing with PDE4 subtypes and CAMP/pCREB signaling, and how the same pathway can cause such contrasting effects in different brain regions.

The results of our study show that PDE4A KO causes anxiogenesis and enhanced emotional memory. Traditionally, most have suggested PDE4 inhibition as the most promising therapy for possible cognitive disorders. However, we feel that this may be an 
oversimplification, and for the first time suggest PDE4A activation as a possible mechanism for anxiolysis and the treatment of emotional memory disorders. While at this time there are no PDE4A specific activators or inhibitors, PDE4B and D specific allosteric inhibitors have recently become available which have much more promise than the previous broad PDE4 inhibitor rolipram (Burgin et al. 2010). As mentioned, cAMP/pCREB signaling has vastly different effects in different brain regions, so in order for PDE therapies to be realistic and successful in the future, further development of PDE4 subtype-specific modulators will be needed.

While the thought of PDE4A activation for anxiolysis is a relatively novel idea, there are data to support this theory. It has been shown that TGF- $\beta$ increases PDE4A expression in the human epithelial type II cell line A549 (Kolosionek et al. 2009), and that mice treated with TGF$\beta$ super-family members displayed anxiolytic-like behavior (Sekiyama et al. 2009). In addition, p70S6 kinase has been shown to activate PDE4A (MacKenzie et al. 1998). Interestingly, separate studies have shown that 5-HT can activate p70S6 kinase (Pullarkat et al. 1998). SSRIs, which are commonly used to treat anxiety, raise extracellular 5-HT levels, and it's possible they may be achieving their effects through the 5-HT/p70S6 /PDE4A pathway. However since SSRIs take several weeks for anxiolytic effects to become evident, if this pathway were involved it's more likely it would be through some alteration of genomic transcription than immediate alteration of functional synaptic strength. Lastly, it has been shown that patients with major depressive disorder (MDD) have a significant reduction of PDE4 expression in the brain (Fujita et al. 2012) as measured by ${ }^{11} \mathrm{C}-(\mathrm{R})$-Rolipram positron emission tomography binding. Interestingly, electroconvulsive shock therapy (ECS) in rats has also been shown to significantly increase the amount of PDE4A, PDE4B, and PDE4D in the striatum and hippocampus (Cho et al. 
2000); unfortunately, this study did not separate the dorsal hippocampus from the ventral hippocampus, which as mentioned previously have different roles in spatial memory and anxiety, respectively (Degroot and Treit 2004). It is important to note that the change in expression of PDE4 observed in MDD patients requires careful interpretation. The decreased expression of PDE4 observed in patients with MDD is may be due to compensatory mechanisms reacting to a decrease of global cAMP signaling (O’Donnell and Xu 2012). In addition, some areas of the brain might benefit from PDE4 inhibition while other areas might benefit from PDE4 activation. As mentioned earlier, PDE4D inhibition is nootropic and antidepressant (Zhang et al. 2002; Li et al. 2011) while overexpression of PDE4D is detrimental to spatial memory (Giorgi et al. 2004). This contrasts the effects seen in PDE4B KO and PDE4A KO, both of which create anxiogenic like profiles (Zhang et al. 2008). Thus, there may be a need to inhibit PDE4D for memory enhancing effects, but activate PDE4A and PDE4B for anxiolytic-like effects. The contrasting roles that PDE4 subtypes play in behavior might account for some of the largely inconsistent findings in regards to PDE4 inhibition and its effects on anxiety-like behavior, as some studies have shown rolipram to be anxiolytic (Silvestre et al. 1999), while others have shown broad PDE4 inhibition to be anxiogenic (Imaizumi et al. 1994; Heaslip and Evans 1995). It is possible that due to the "messy" non-specific inhibition of all PDE4 subtypes under rolipram, multiple phenotypes can emerge due to the contrasting roles the PDE4 subtypes may play in behavior and various brain regions. This highlights the incredible complexities in the CAMP signaling system that need to be overcome for the development of future PDE drug targets to be specific, practical, and efficacious. 
Also, while mice deficient in PDE4D display emetic-like behavior (Robichaud et al. 2002b; Li et al. 2011), which is the major side effect of PDE4 inhibitors (Robichaud et al. 2001; Dyke and Montana 2002; Robichaud et al. 2002a), PDE4A KO mice did not show any emesis-like effects as demonstrated by unaltered duration of anesthesia mediated by alpha2-adrenergic receptors, a surrogate measure of emesis in rodents (Correa-Sales et al. 1992; Robichaud et al. 2002b). The differential contributions of PDE4A and PDE4D to emetic responses appear to be attributed to the unique distribution pattern of the PDE4 subtypes in emesis-associated brain regions, including the area postrema and the nucleus of solitary tract, in which PDE4D is highly expressed, but PDE4A is barely expressed (Cherry and Davis 1999; Lamontagne et al. 2001; D’Sa et al. 2005; Mori et al. 2010).

Lastly, it is important to consider the potential confounding variables which we can't exclude in this study. For example, one intrinsic drawback to using global PDE4A KO is the inability to reduce expression in a time-specific or tissue-specific manner. Since PDE4A is knocked out from conception, we were unable to rule out the potential alterations in development that might occur due to the knockout of PDE4A. Also, we are unable to rule out the effects of compensatory changes from the remaining PDE4 subtypes. While previous reports have shown no compensatory changes in PDE4-subtype KO mice (Zhang et al. 2002; Zhang et al. 2008), the PDEs are known to be incredibly compensatory (O'Donnell and Xu 2012). In addition, because PDE4A is expressed in the periphery as well as the CNS, we can't exclude the potential effects peripheral manipulation might have on the brain regions inducing behavior. Viral-specific PDE4A knock-down of adult WT mice in discrete brain regions, or the development of a Tet on/off Cre-lox PDE4A system would ideally be the best option for 
studying PDE4A, and development or utilization of these technologies should be used in the future to confirm these studies and further characterize the role of PDE4A in behavior.

\subsection{Summary}

In conclusion we have shown for the first time that PDE4A KO results in an anxiogenic phenotype, an increase of corticosterone post stress, and enhancement of emotional memory.

In addition, we did not notice a change in the duration of anesthesia with PDE4A KO, suggesting it could be a possible drug target that could avoid emetic effects plaguing many PDE4 inhibitors. Further research is needed to identify the mechanisms with which this takes place; however, we hypothesize the effects of PDE4A KO are most likely due to elevations of CAMP levels in anxiety-related brain regions such as the amygdala, ventral hippocampus, and the dorsal PLmPFC. PDE4A KO could result in hypoactivity of the dorsal PL-mPFC through activation of HCN channels which should hyperpolarize the pyramidal neurons resulting in decreased firing and suppressed inhibition of the PVN and HPA axis. In addition, elevations of cAMP levels could strengthen the amygdala by increasing spinogensis and hyperexcitability, possibly through an elevated pCREB-BDNF neurotrophic signaling pathway. Lastly, PDE4A KO could result in increased hippocampal theta rhythm which has been shown to be elevated in anxiety-like behavior. The combination of these factors would be synergistic on anxiogenesis, as there would be decreased inhibition, and increased excitability of the stress response. These speculations match what is seen in a clinical setting, as patients with general anxiety disorder have been observed to have hyperactivity in the amygdala, and hypoactivity in the mPFC (Russo et al. 2012). In addition, we suggest for the first time that specific PDE4A activation as opposed 
to broad PDE4 inhibition could be a potential therapeutic target in the future not only for the treatment of anxiety disorders, but perhaps negative emotional memory disorders as well.

It was noted that PDE4A KO did not alter the baseline of FST or TST behavior relative to WT controls, in contrast to PDE4D KO, which produces antidepressant-like behavior (Zhang et al. 2002). However, this does not mean PDE4A is not involved in antidepressant activity given that its levels in the brain are highly responsive to antidepressant treatment (Ye et al. 2000; D'sa et al. 2005). Further studies with chronic mild stress may help to clarify this.

\subsection{Contributions}

Rolf Hansen performed all of the above behavior work, PDE4A western blots, and corticosterone and T3 ELISAs. Dr. Han-Ting Zhang and Rolf Hansen were both involved with the design of experiments. Dr. Qiang Wu performed the cAMP ELISAs and pCREB western blots. Dr. Visvanathan Ramamurthy and Saravanan Kolandaivelu performed the mouse ERG studies.

Additional thanks to: Dr. Marco Conti for the PDE4A KO mice, Dr. James O'Donnell for his guidance and use of laboratory materials necessary for this research to be performed, Dr. James O'Callaghan for the assistance and use of his microwave fixation machine, Dr. Rae Matsumoto for the use of her PAS automated open field chambers, and Dr. Max Sokolov for the use of his Licor Odyssey Infrared Imaging Scanner. This work was supported by research grants from the NARSAD Young Investigator Award [1008640 for 2006, and 10011226 for 2008 to H.T. Zhang], and the National Institute on Aging [AG031687 to H.T. Zhang].

Portions of this chapter were taken from previously published manuscripts (Hansen and Zhang 2013; Hansen et al. 2014). 
Chapter 3

PDE4A KO and Obesity 


\subsection{Introduction}

Obesity is a disorder that has risen to the ranks of national pandemic affecting $~ 30 \%$ of the population, and costing the United States over 150 billion dollars a year (Ogden et al. 2012). Obesity is also major risk factor for leading causes of death such as cardiovascular disease, stroke, and diabetes. Obesity is defined as a body mass index of over $30 \mathrm{~kg} / \mathrm{m}^{2}$, or a body fat percentage over $25 \%$. Sadly, obesity is one of the most preventable disorders in the country; however, due to cultural changes such as increased work load, decreased time for exercise, and overabundance of affordable easily obtainable trans-saturated fats, it has become a pandemic to which our country is currently losing. These statements are made evident by the fact that zero states have been able to reduce their obesity rating below $15 \%$ as set forth in the United States healthy people 2010 pledge(Services 2010).

Obesity can be caused by many things in addition to lack of exercise and poor nutritional intake such as drug side effects (SSRIs, antihypertensives, steroids), diseases (hypothyroidism, Cushing's syndrome, depression, anxiety), or genetics (heritability of obesity or high BMI has been predicted to be as high as 84\%) (O'Rahilly and Farooqi 2006). Because of this, there truly is a need to develop new therapies since obesity is not always preventable, or able to be attenuated by lifestyle changes. In addition, while much is known about the hormonal patterns involved with obesity, there is still much to be characterized regarding CNS-mediated neural correlates.

Obesity is a complex, multifactorial disease that can be influenced by a number of different body systems and variables. When trying to identify the mechanisms by which a 
variable affects adiposity, one must take many things into consideration. This includes hormonal control exerted by the central nervous system and peripheral organs, adipogenesis, adipolysis, adipocyte size, exercise or baseline movement, food consumption, and metabolic rate to name a few. For example, it is well known that leptin (a satiety signal secreted from adipocytes) can decrease appetite and adipogenesis through inhibition of the neuropeptide $Y$ (NPY) and Agouti-related peptide (AgRP) which reside in AgRP/NPY neurons in the arcuate nucleus of the hypothalamus. Leptin KO mice (ob/ob mice) become severely obese, mostly due to uncontrolled eating resulting from a lack of satiety signal. Interestingly, in humans, obesity is often associated with extremely high levels of leptin characteristic of "leptin resistance" putatively due to a loss of sensitivity to leptin resulting in dysregulation of homeostaticfeedback mechanisms.

There are many treatments or ways to prevent obesity including routine exercise, living an active lifestyle, and most importantly making healthy lifestyle choices such as eating nutritionally well balanced meals, and avoiding trans-saturated fats. When obesity reaches dangerous or life threatening levels, more extreme therapies are also available such as gastric bypass surgery, which is effective but expensive, and can have severe consequences such as hemorrhaging, bowel obstructions, infection, anastomotic leakage, dumping syndrome, ulcers, hernias, nutritional deficiencies, and even death. Anti-obesity drugs such as orlistat (inhibits pancreatic lipase), sibutramine (selective serotonin-norepinephrine reuptake inhibitor), rimonabant (cannabinoid 1 receptor antagonist), metformin (AMP-activated protein kinase activator), exenatide (GLP-1 analogue), and pramlintide (amylin analogue) are available however they have severe drawbacks. Many of them have severe health concerns and have been pulled 
by the FDA (sibutramine, rimonabant), while other treatments are approved only for use in diabetes related obesity (meformin, exenatide, and pramlintide). Currently the only FDA approved therapy for obesity is Orlistat, which is a pancreatic lipase inhibitor. The simple fact that obesity prevalence is significantly increasing every year indicates a need for additional therapies to help alleviate this disorder; phosphodiesterases may represent a novel therapeutic target for combating obesity.

The role that CAMP and PDEs play in obesity is beginning to be uncovered; however much more research is needed in order to characterize the complex regulation cAMP has on adiposity. It is well known that PDE3B plays a very large role in insulin-mediated lipogenesis (Omar et al. 2011). This is largely due to the activation of PDE3B by insulin, which results in a subsequent decrease of CAMP and lowered activation of hormone sensitive lipase, which ultimately decreases lipolysis in adipocytes. However, it is unreasonable to assume an overall activation of PDEs results in a lipogenic profile since PDEs have an extensive variation of expression and functional roles in the body. Interestingly, inhibition of PDE1C, PDE3B, PDE4C, and PDE8B resulted in increases of insulin secretion from rat pancreas (Waddleton et al. 2008). Also, inhibition of PDEs results in a decrease of glucose uptake by adipocytes (Mors-Fletchtner et al. 2005). It is important to remember that chronically high levels of glucose in the blood stream can lead to insulin resistance. Thus, lowered PDE activities in individuals, and subsequently elevated cAMP levels, could possibly lead to insulin resistance due to chronically elevated insulin release and high glucose levels. In agreement with this is the finding that PDE4 activity is significantly reduced in obese mice (Greene et al. 2009). Furthermore, it has been shown that PDE4 activity is significantly reduced in the adipocytes of obese individuals (Omar et al. 2011). 
Since it appears that PDE4 activity may be decreased in obese individuals, one would expect the downstream effects of cAMP to be increased. Moreover, it has been shown that CREB activation is significantly elevated in obese mice (Qi et al. 2009). CREB activation induces adipogenesis in 3T3-L1 cells (Reusch et al. 2000), and mice fed a high fat diet have elevated levels of PCREB and BDNF in the striatum, which is correlated with a strong anxiogenic and depressive profile (Sharma and Fulton 2012). The mechanism behind this rise in activated CREB may also be more than just elevated cAMP signaling, as it has been shown that mice fed a high fat diet also have significantly lower levels of the CREB inhibitor ICER. However, there are also paradoxical publications that show PDE4 inhibition induces weight loss through increased lipolysis (Nakamura et al. 2004; Snyder et al. 2005). The effect of PDE4 inhibitors to induce lipolysis may be mediated by PDE4B, which has been shown to be expressed at levels more than twice as high compared to PDE4A in adipocytes (Wang and Edens 2007). Supporting this is the evidence that PDE4B KO mice are leaner than WT littermates, and protected against obesity (Zhang et al. 2009) This fact once again details the incredible complexity and functional roles that different PDE4 subtypes might have, and the lack of specificity able to be achieved by broad PDE4 inhibitors. This leads to the objective of specific aim 2, to identify the role that PDE4A plays in obesity. Our hypothesis is that decreasing PDE4A expression and activity will be lipogenic. The rationale behind our hypothesis is that PDE4 activity is strongly reduced in obese individuals, and downstream molecules such as PCREB and BDNF are elevated. In addition, it has also been shown that inhibition of adenylyl cyclase $V$, which results in less CAMP, is protective against a high fat diet (Ho et al. 2011). One additional piece of evidence is the fact that inhibition of PDE4A5 resulted in a significant potentiation of growth hormone induced adipogenesis (MacKenzie et al. 1998) Interestingly, this 
study also showed that PDE4A5 is capable of being activated by p70S6 kinase. Leptin has also been shown to activate P70 S6 kinase, indicating that PDE4A5 may be a downstream effector of leptin mediated satiety and thermogenesis. In agreement with this is the fact that leptin has also been shown to reduce CAMP in the NPY neurons of the arcuate nucleus, while ghrelin has the opposite effect (Zhao 2005). While it is known this is mediated through PDE3B, the role of PDE4A cannot be ruled out.

We performed several experiments in order to characterize the role PDE4A may play in obesity. First, we used PDE4A KO mice to determine the effect of PDE4A depletion on baseline weight gain. Second, we determined if PDE4A KO affects food consumption. Lastly, we examined the effects of PDE4A KO on baseline metabolism by assessing the levels of T3 hormone present in the blood of PDE4A KO mice vs WT controls.

\subsection{Materials and Methods}

\section{Animals}

All procedures were approved by the Animal Care and Use Committee of West Virginia University Health Sciences Center and performed according to the recommendations of the NIH Guide for the Care and Use of Laboratory Animals 7th edition (NIH Publications No. 80-23, revised 1996). Adult male PDE4A KO mice on a C57 background were generated by homologous recombination and heterozygote breeding as already described previously (Jin et al. 1999; Jin and Conti 2002; Ariga et al. 2004; Jin et al. 2005a; Bruss et al. 2008; Jin et al. 2010). In brief, the PDE4A gene was disrupted though replacement of a $3 \mathrm{~kb}$ region encoding exons 10 14 using a PGK-hrpt cassette in the reversed orientation via homologous recombination. These 
PDE4A KO mice are commercially available through the Mutant Mouse Regional Resource Centers which also provides additional details on their generation (MMRRC;

http://www.mmrrc.org; Stock number 034793-UCD). Age-matched (4-8 months) wild-type (WT) mice (C57BL/6J) of the same background were used in this study. All genotyping was confirmed by Southern blot analysis with tissues obtained via tail clipping and subsequent detection of genomic DNA. Animals were housed in groups of 1-5 per cage (Allentown XJ cage, $12.5^{\prime \prime}$ length $\times 6$ " width $\times 5^{\prime \prime}$ height, 75 square inches of floor space) in a light (12 h on, 12 h off; lights on at $0600 \mathrm{~h}$ ) and temperature controlled room $\left(22-23^{\circ} \mathrm{C}\right)$. Water and food were available ad libitum

\section{Locomotor activity}

Mice were placed into individual PAS automated open field chambers (San Diego Instruments San Diego, CA). The number of light beam crossings for locomotor activity (horizontal crossings) and rears (vertical crossings) were recorded and averaged over six 5-min intervals (Hu et al. 2011).

\section{Food Consumption}

To monitor the effect of PDE4A KO on food intake, the levels of food were monitored for 3 days. Mice were habituated to individual housing over the course of several days. After several days, food intake was assessed by weighing the food each consecutive morning for 3 days.

\section{T3 Levels}


To determine the effect of PDE4A KO on metabolism, the levels of T3 hormones were

measured. Blood was collected into a heparin tube by using a mouse $5.5 \mathrm{~mm}$ goldenrod animal lancet to puncture the submandibular vein. Blood plasma was then separated via centrifugation and subsequent decanting of the plasma layer. Plasma T3 levels were obtained via a T3 ELISA (CALBIOTECH, cat\# T3043T-11).

\section{Statistical Analysis}

All data shown are means \pm SEM, and were analyzed using the GraphPad Prism 5 software (version 5.04). Locomotor activity was analyzed via 2-way ANOVA with repeated measures and Bonferroni's post test. Food consumption, weight, and T3 levels were analyzed via students two-tailed T-tests. Alpha significance was achieved with a $P$ value of $<0.05$.

\subsection{Results.}

\subsubsection{PDE4A KO and weight gain}

To determine the effect of PDE4A KO on weight gain, the body weights of PDE4A

KO mice and WT controls were measured

up until 8 months of age when the

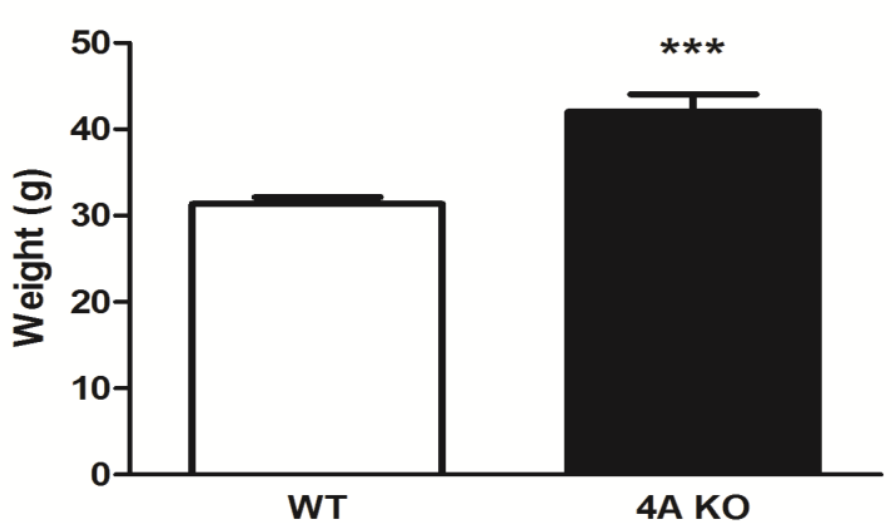

behavior tests ceased. It was discovered that PDE4A KO mice had a significantly higher body

weight than WT controls $(t=5.010, P=.0002$;

Figure 3.1) starting around 3 months of age.

This weight plateaued and remained the same
Figure 3.1. The effect of PDE4A KO on weight gain. PDE4A KO mice weighed significantly more than WT controls. The data displayed represents the average weight of the mice from 3-8 months of age when the weight was observed to plateau. 
until the animals were sacrificed around 8 months of age.

\subsubsection{PDE $4 A K O$ and locomotor activity}

To determine the effect of PDE4A KO on locomotor activity, animals were placed into a PASautomated open-field chamber and the number of light crossings recorded and averaged over six 5-min intervals. PDE4A KO mice and WT controls displayed no significant difference in locomotor activity, and light beam crossings for both groups decreased significantly over time (2-ANOVA-R: significant effect of time, $F_{(5,130)}=16.57, p<0.0001$; no effect of genotype, $F_{(1,26)}=$ 2.19, $p=0.151 ;$ no effect of genotype $x$ time interaction, $F_{(5,130)}=0.75, p=0.585$; Figure 2.2a). As an additional measure of exploratory activity, the number of rears was also recorded while the mice were in the open-field chambers. It was found that PDE4A KO mice did display significantly lower rearing levels than WT controls during the first 15 min of the test (2-ANOVA-R: significant effect of PDE4A KO, $F_{(1,26)}=12.77, p=$ 0.0014 ; significant effect of time $x$ genotype interaction, $F(5,130)=2.36, p=$ 0.0438 ; no effect of time, $F_{(5,130)}=$ 2.27, $p=0.052$; Figure $2.2 b)$. This data indicates that while PDE4A KO did not induce lethargy as interpreted by a lack of

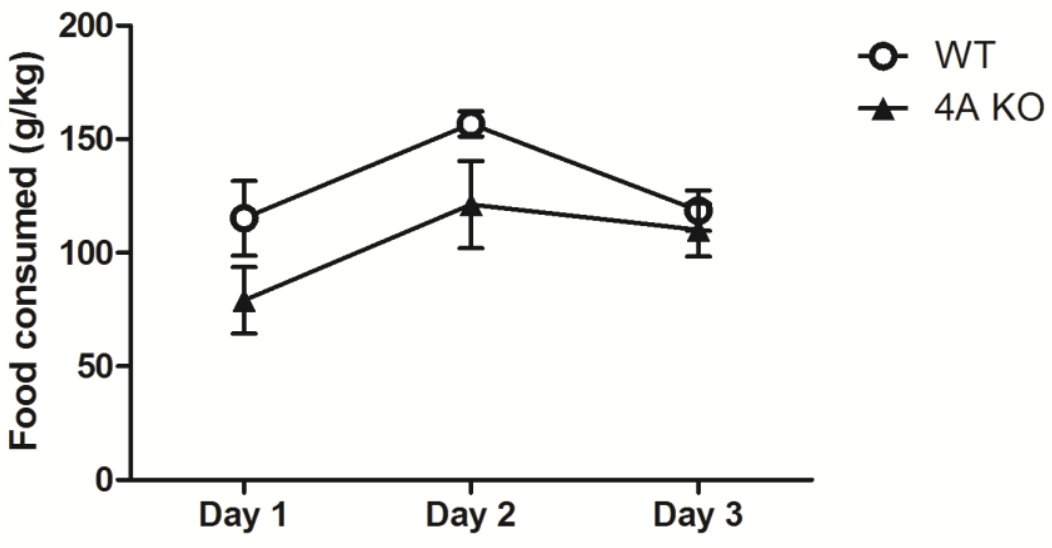

Figure 3.2. Effects of PDE4A KO on food consumption. PDE4A KO mice displayed no changes in food consumption compared to WT controls. Data shown represent means \pm SEM; $n=9$ /group; (two-way ANOVA with Bonferonni multiple comparison tests).

changes in horizontal light crossings, there was a decrease in vertical exploratory rearing. 


\subsubsection{PDE4A KO and food consumption}

To determine if the change in weight displayed with PDE4A KO was due to hyperphagia, food intake was monitored in PDE4A KO and WT mice for 3 days. It was found that PDE4A KO mice did not display elevated levels of food consumption when compared to WT controls, and actually ate significantly less total food when normalized for body weight (2-ANOVA-R: significant effect of PDE4A KO, $F_{(1,14)}=6.42, p=0.0238$; no effect of time $x$ genotype interaction, $F_{(2,28)}=.56, p=0.5765 ;$ significant effect of time, $F_{(2,28)}=4.08, p=0.0278 ;$ Figure 3.2).

\subsubsection{PDE4A KO and T3 levels}

To determine if the effects of PDE4A KO on

weight gain were due to changes in metabolism, we investigated levels of the main metabolic hormone T3 in PDE4A KOs vs WT controls. In order to ensure that any change observed in PDE4A KO animals was specific to PDE4A, we also investigated the levels of T3 in PDE4B KO mice and WT littermates. PDE4A KO animals displayed significantly lower levels of T3

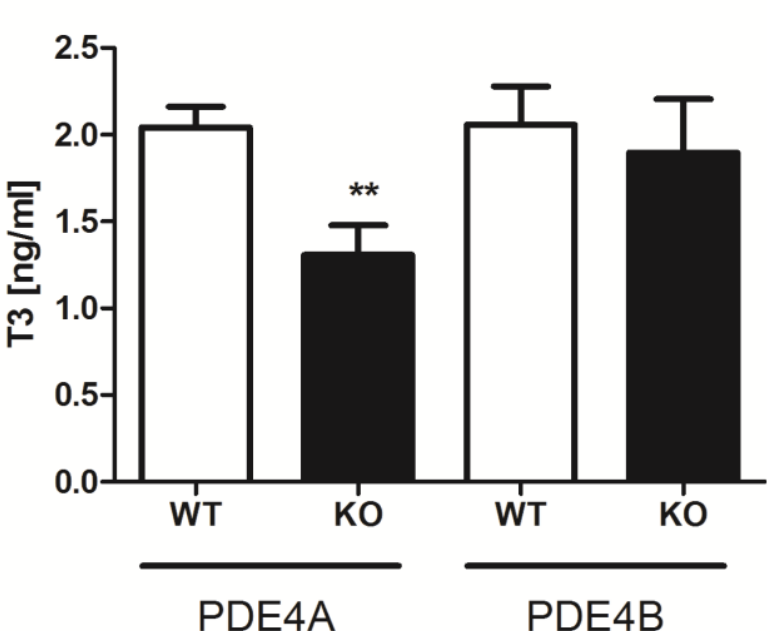

Figure 3.3. Effect of PDE $4 A$ KO on T3 levels. PDE4A KO mice displayed significantly lower T3 levels than WT controls. In addition, this decrease of T3 was specific to PDE4A KO as

hormone than WT controls animals $(t=3.536, \mathrm{P}$ PDE4B KO mice did not display decreased levels of T3 hormone. Data shown represent means $\pm S E M ; n=9 /$ group;

$=.0054$ Figure 3.3$)$. This suggests that PDE4A

KO may alter baseline metabolism, and this effect is specific to PDE4A and not PDE4B. 


\subsection{Discussion.}

In this study we have shown that PDE4A KO results in an obesogenic phenotype. PDE4A KO mice were significantly heavier than WT controls, upwards of $45 \mathrm{~g}$ compared to the average of 30g for WT mice. The next goal was to determine a possible explanation for this weight gain. There are many possible avenues for obesity to occur such as: decreased activity, increased food consumption, decreased metabolism, increased adipogenesis, increased absorption of fats and nutrients, and increased baseline adipocyte size. To determine if changes in activity were causing the obesity, PDE4A KO mice were subjected the open field locomotor test. It was observed that PDE4A KO mice displayed no significant changes in activity over a 30 minute period. This data suggests that changes in activity levels are not responsible for the observed obesity in PDE4A KO mice. Additional home cage baseline activity tests in the future however should be used to confirm these findings (i.e. use of a home cage monitoring system). The next possible cause for obesity that we tested was food consumption. It has been shown that mice with alterations in leptin signaling (one of the main satiety hormones) become severely obese due to hyperphagia and hypometabolism. Essentially these ob/ob mice have no satiety signals and excessively consume food until they become morbidly obese. To test for hyperphagia, we acclimated the animals to single housing. After single housing, the amount of mouse chow in the cages was weighed every day to determine the amount of food being ate. Surprisingly, PDE4A KO mice showed no significant changes in food consumption compared to WT controls over the course of 3 days, suggesting that food consumption was not one of the major mechanisms responsible for the obesogenic profile in these animals. 
Next, we decided to examine the effects of PDE4A KO on triiodothyronine hormone (T3), one of the major metabolic hormones of the body. T3 exerts these effects through a number of mechanisms such as glycogen breakdown and lipolysis. In order to show that any possible effects of PDE4A KO on T3 were specific, we also decided to look at PDE4B KO mice T3 levels. T3 ELISA absorptions determined that PDE4A KO mice had significantly lower T3 levels than WT controls. In addition, this effect was specific to PDE4A KO mice, as PDE4B KO mice did not display any change in T3 levels. These findings suggest that PDE4A KO therefore may induce obesity through changes in the metabolic hormone T3, and the subsequent basal metabolic rate of the body. This is not believed to be a compensatory change, as in the clinic obese individuals typically have elevated T3 levels, which is perhaps a response of the body to try to decrease their weight (Faber et al. 1981). The T3 levels also shed light onto a possible relationship between the anxiogenic phenotype observed in PDE4A KO mice (see chapter 2). As mentioned, it has been shown that anxiety, depression, and obesity are highly co-morbid in the human population (Simon et al. 2007), a result that was also observed in the PDE4A KO mice phenotype. The relationship between stress, anxiety, and T3 levels is a complicated one, and additional research is needed (Faber et al. 1981; Williamson and Davison 1987; Munjack and Palmer 1988; Byerly et al. 2009). However, it has been shown that stress and anxiety in rats can result in hypothyroidism (Olivares et al. 2012) and lowered T3 levels (Helmreich et al. 2005; Helmreich and Tylee 2011). Thus, it seems rationale to assume that the strong anxiogenic phenotype observed in PDE4A KO mice could have attenuated the release of T3, and resulted in the subsequently observed obesity. Interestingly, it has also been shown that mice with decreased thyroid signaling display anxiogenic behavior, and that supplementation with T3 
reversed this phenotype (Venero et al. 2005; Buras et al. 2014). Thus it appears that the influence of anxiety on T3, and conversely T3 on anxiety, could be cyclical, with a positive feedback loop occurring in the PDE4A KO mice.

While we believe that changes in baseline metabolism due to changes in T3 levels may be a primary cause for the obesity in PDE4A KO mice, there are other mechanisms we have not tested that could also be playing a role. One additional explanation for the obesity observed in PDE4A KO mice could be due to increased adipogenesis. While there is very little research on the regulation of PDE4, CAMP, and obesity, there is one study which shows that in preadipocyte-3T3-F442A fibroblast cells, antisense deletion of PDE4A5 resulted in a higher differentiation rate of fibroblasts into adipocytes. Conversely, it was shown that growth hormone induced activation of PDE4A5 served as a "brake" for the differentiation process; particularly, PDE4A5 was specifically activated by P70S6 Kinase (MacKenzie et al. 1998). Thus in this "model of adipogenesis", it appears that depletion of PDE4A5 leads to increased "adipogenesis", and activation of PDE4A5 led to lowered adipogenesis rates. This in vitro data, while not causal, is consistent with what we observed in PDE4A KO mice. If depletion of PDE4A5 does cause increased adipogenesis, then this is one mechanism through which the PDE4A KO obese phenotype could be explained. Leptin has also been shown to activate P70S6 kinase, indicating that PDE4A5 may be a downstream effector of leptin mediated satiety and thermogenesis. In agreement with this is the fact that leptin has also been shown to reduce cAMP in the NPY neurons of the arcuate nucleus, while ghrelin has the opposite effect (Zhao 2005). While it is known that this is mediated through PDE3B, a role for PDE4A cannot be ruled out. 
Additional studies have also provided evidence that increased cAMP signaling (decreased PDE4 activity) may be a possible mechanism of obesity. It has been shown that PDE4 activity is significantly reduced in both obese mice (Greene et al. 2009) and adipocytes from obese humans (Omar et al. 2011). Since it appears that PDE4 activity may be decreased in obese individuals, one would expect the downstream effects of cAMP to be increased. Moreover, it has been shown that CREB activation is significantly elevated in obese mice (Qi et al. 2009). CREB activation induces adipogenesis in 3T3-L1 cells (Reusch et al. 2000), and mice fed a high fat diet have elevated levels of pCREB and BDNF in the striatum, which is correlated with a strong anxiogenic and depressive profile (Sharma and Fulton 2012). The mechanism behind this rise in activated CREB may also be more than just elevated CAMP signaling, as it has been shown that mice fed a high fat diet also have significantly lower levels of the CREB inhibitor ICER. It has also been shown that inhibition of adenylyl cyclase $\mathrm{V}$, which results in less cAMP, is protective against a high fat diet (Ho et al. 2011).

While there have been many studies suggesting that increased CAMP is obesogenic, there have also been many studies showing that PDE4 inhibition actually protects against high fat diets (Nakamura et al. 2004; Snyder et al. 2005; Park et al. 2012; Doseyici et al. 2014). However, it is important to remember the incredible complexity and compartmentalization of CAMP and PDE signaling (Houslay et al. 2007; Lynch et al. 2007; Baillie 2009; Houslay 2010). While PDE4A KO mice in our study were obese, it was previously shown in other labs that PDE4B KO may be protective against obesity (Zhang et al. 2009). It is not surprising that broad PDE4 inhibition with a drug throughout the entire body would have significantly different effects than local inhibition of a single splice variant in one region of the body. 
An additional mechanism for which we were unable to test for was altered absorption of fat and nutrients in PDE4A KO mice. This could be broken down into initial absorption of fats and nutrients in the GI tract, as well as cellular uptake of glucose and fat. In regards to the absorption of nutrients in the GI tract, the microbial flora found in the small intestine and colon play a significant role in this absorption. Mediation of inflammatory factors and the immune response could have significant impacts on the characteristic of these microbial flora. It has been shown that PDE4B regulates the release of certain inflammatory factors, thus it is a possibility that PDE4A could also be playing such a role. However this possibility is more unlikely as PDE4B was suggested as the primary PDE4 subtype responsible for the mediation of this response (Jin and Conti 2002).

\subsection{Contributions}

Rolf Hansen performed all of the above work. I would like to thank Dr. Han-Ting Zhang for the supervision and Dr. Stanley Hileman for the advice, Dr. Marco Conti for the PDE4A KO mice, Dr. James O’Donnell for his guidance and use of laboratory materials necessary for this research to be performed and Dr. Rae Matsumoto for the use of her PAS automated open field chambers. This work was supported by research grants from the NARSAD Young Investigator Award [1008640 for 2006, and 10011226 for 2008 to H.T. Zhang], and the National Institute on Aging [AG031687 to H.T. Zhang]. 


\section{Chapter 4}

\section{Conclusions and Future}

\section{Studies/Directions}




\subsection{Conclusions}

The goal of this dissertation was to characterize the functional role that PDE4A plays in both behavior and obesity. In summary, this dissertation has shown that PDE4AKO:

$>$ Displays no effect on locomotor activity, but significantly reduced rearing levels

Induces anxiety-like behavior as measured by the elevated plus maze, hole board, lightdark transition, and novelty-suppressed feeding tests

- This change in anxiety-like behavior also correlated with prolonged and elevated levels of corticosterone in PDE4A KO mice in response to food-deprivation stress

Displays no change in depressive-like behavior as measured by the forced-swim and tailsuspension tests

$>$ Improves "emotional memory" as measured through step-through passive avoidance

Has no effect on "episodic-like" memory in the novel object recognition test

Impairs spatial memory training in the Morris water maze, however retrieval appeared unaffected

Increases CAMP and PCREB levels in the hippocampus and frontal cortex

PDE4A KO is obesogenic 
- This gain in weight was not due to differences in activity or food consumption, and was most likely metabolic as T3 hormone levels were significantly reduced in PDE4A KO mice

These studies have significantly improved our knowledge of the PDE4A subtype and its functional role in the body. However, further studies are needed to characterize the mechanisms by which PDE4A modulates anxiety, emotional memory, and obesity.

\subsection{Future Studies and directions}

While this dissertation has begun to characterize the functional role that PDE4A plays in behavior and obesity, we have yet to identify the exact mechanisms by which it does so. The following studies would significantly further our knowledge of the mechanisms behind PDE4A KO and anxiety/obesity.

\subsubsection{Investigating the effects of stress and anxiety on PDE4A expression and activity. In this} dissertation we have shown that PDE4A KO mice display a strong anxiogenic phenotype. Due to the fact that loss of PDE4A expression promotes anxiety-like behavior, it is reasonable to suggest that inducing stress and anxiety in WT mice would alter PDE4A expression and activity in anxiety-related regions of the body. Our hypothesis is that PDE4A expression and activity would be decreased after stress in anxiety related brain regions such as the prefrontal cortex, amygdala, and hippocampus. The rationale behind our hypothesis is that depletion of PDE4A induces anxiety, so it is logical to assume that PDE4A expression and activity would have an inverse relationship. This is supported by evidence that elevation of cAMP in the amygdala 
correlates with anxiety. We propose that WT C57 mice should be restraint stressed for various periods of time (1-10) days. The effects of stress and anxiety on PDE4A expression/activity would be looked at during following time points after the stress (immediately after, hours after, day after, etc.) This paradigm would allow the researcher to determine the immediate effects of stress on PDE4A expression and activity, as well as long term changes. Sampling the effects of stress on PDE4A function at multiple time points also offers a better insight into the time course of any changes observed. PDEs are notorious for being incredibly compensatory, so it's possible that PDE4A levels could have opposite expression patterns in acute vs chronic stress. Sampling at multiple time points would allow all these patterns to be observed. Supporting this theory, it has been shown that after unpredictable foot shock stress, PDE4A1, PDE4A5, and PDE4A10 mRNA transcripts are decreased in the basolateral nucleus of the amygdala (Stair and Rainnie 2011). While this study is an important first step in agreeing with our theory that increased cAMP signaling in the amygdala correlates with anxiety, these findings need confirmed with western blot and protein levels. In addition, this study has not ruled out whether the decreased expression of PDE4A mRNA transcripts is compensatory, or whether these decreased levels are directly causing the anxiety-like behavior observed. Additional studies explained below could help to answer these questions.

\subsubsection{Can the PDE4A KO phenotype be rescued by overexpression of PDE4A? While the PDE4A} KO mice we studied displayed significant changes in obesity and anxiety, global KO mice have their limitations. First, we are unable to rule out the effects of development on behavior. It is possible that PDE4A KO changed the formation and connections of the brain which could inherently result in changes in behavior and obesity. In addition, we are not able to rule out 
indirect changes of PDE4A KO on behavior and obesity. Rescue studies are strongly suggested when further trying to validate the results seen in a knock out study. In order to perform this experiment, PDE4A would need to be overexpressed in the brains of PDE4A KO mice. This could be done through lentiviral vectors infused into various parts of the brain we believe could be responsible for the manifestation of anxiety, emotional memory, and obesity such as the prefrontal cortex, amygdala, hippocampus, and hypothalamus. If the phenotype of PDE4A KO mice were able to be reversed through the overexpression of PDE4A, it would further validate our hypothesis on the specific role of PDE4A in these conditions.

\subsubsection{Does PDE4A inhibition and overexpression in WT mice modulate anxiety-like behavior and} obesity? While there are currently no specific PDE4A activators or inhibitors available, overexpression and inhibition of the PDE4A enzyme could be performed through viral vectors carrying dominant negatives and overexpression constructs. These viral vectors are currently available and ready for use. Specifically increasing PDE4A levels, or inhibiting the PDE4A enzyme would answer many questions. First, by inhibiting the enzyme or increasing its presence in adult mice, the confounding effects of development can be ruled out. In addition, this study would also be able to rule out the possible confounding variable of peripheral effects. PDE4A is not expressed uniquely in the brain, it is also in other areas of the body such as the adrenal cortex, thyroid gland, pancreas, and adipocytes which could cause changes in these areas indirectly causing the anxiety-like behavior and obesity observed in the PDE4A KO mice. For example, while we hypothesized that the amygdala is being over activated by elevated cAMP levels due to loss of PDE4A, it is possible that the adrenal cortex is being changed by loss of PDE4A, and subsequent cAMP signaling as well (Bourdeau and Stratakis 2002). If PDE4A KO 
were to cause more corticosterone to be released from the adrenal cortex, this could possibly be responsible for the change in behavior we observed. This would be a very powerful study because it would specifically show the causal relationship between PDE4A and the results we observed in PDE4A KO mice. Also, this study would be able to narrow down which PDE4A splice variants are relevant for anxiety, as well as which brain regions are playing a role.

4.2.4. The effect of HCN channels on PDE4A and anxiety. As mentioned earlier, HCN channels in the mPFC could be playing a significant role in the manifestation of anxiety-like behavior observed in PDE4A KO mice. To further characterize the CAMP pathway involved with this behavior, HCN channel inhibitors could be infused into the MPFC of PDE4A KO mice. We hypothesize that inhibition of HCN channels in PDE4A KO mice would attenuate the anxiety-like behavior observed and partially rescue the PDE4A KO anxiogenic phenotype.

\subsubsection{Further characterizing the causal relationship between PDE4A KO and obesity. We}

observed in these studies that PDE4A mice display both significant anxiety-like behavior, and obesity. These findings are consistent with the high comorbidity of anxiety and obesity observed in the human population. While we were able to document and record these findings, we are still uncertain of the causal relationship between the two. It is very possible that increased anxiety-like behavior is stressing the body, causing inflammation and subsequent alterations in adipocyte characteristics as well as changes in the base-line metabolism we observed as a decrease of T3 hormone. In addition, it is also possible that the obesity appeared first and that this stress on the body caused increased anxiety-like behavior both through direct and indirect mechanisms. Further characterization of this relationship would significantly help to identify the mechanisms with which PDE4A modulates these conditions. One experiment 
could be to give anxiolytics such as BZDs or SSRIs to PDE4A KO mice at a young age to see if they could prevent the significant weight gain observed. An alternate approach could be to more closely monitor PDE4A KO mice for behavior at a younger age to try to illicit which characteristic appears first. To determine if the obesity is affecting the anxiety, PDE4A KO mice could have their diet supplemented with replacement T3 hormone. After a period of time, the change in anxiety-like behavior and obesity levels could be measured. These studies are further supported by literature showing that stress and anxiety can decrease thyroid function (Helmreich et al. 2005; Helmreich and Tylee 2011; Olivares et al. 2012), and that decreased thyroid function can also cause stress and anxiety (Venero et al. 2005; Buras et al. 2014).

\subsection{Summary}

With these initial studies, and further characterization of the mechanisms by which PDE4A modulates anxiety-like behavior and obesity, we believe that PDE4A could someday be a potential target for anxiety, emotional memory disorders, and obesity. PDE4A KO had no effect on locomotor activity, therefore we believe that targeting PDE4A as an anxiolytic could avoid the side effects of sedation and lethargy which plagues BZDs. In addition, we believe that it is important to recognize the incredible complexity of cAMP/PDE signaling and that inhibition of PDEs may not be the appropriate therapy in all scenarios. With the previous sections in mind, one can recognize the incredibly delicate balance of cAMP signaling that occurs in the brain, and that global activation of cAMP through broad PDE4 inhibition might not be the best approach. As mentioned, in regards to WM it appears that decreases in cAMP levels in the PFC would actually improve memory. This also might hold true in the nucleus accumbens and 
amygdala, as overexpression of CREB in these region has been shown to produce anhedonia (Muschamp et al. 2011) and anxiety (Wallace et al. 2004), respectively.

There appears to have been a "herd mentality" in the field of PDE4 research, as PDE4 inhibition is consistently talked about as if it is the only mechanism through which PDE4 modulation could have beneficial effects. However, this is starting to appear too simplistic, and there may need to be a paradigm shift in the way we think about PDE4 modulation as a therapeutic for cognitive disorders; in particular, researchers may need to begin thinking about PDE4 activation as a possible therapeutic strategy for the future. This makes particular sense in regards to anxiety or working memory. However, it appears in regards to hippocampusdependent memory that PDE4 inhibition may still be the best approach.

\subsection{Contributions.}

Portions of this section were taken from a soon to be published review "Phosphodiesterase-4 modulation as a potential therapeutic for cognitive loss in pathological and non-pathological aging: Possibilities and Pitfalls". 


\section{References}

Adamec R, Hebert M, Blundell J (2011) Long lasting effects of predator stress on pCREB expression in brain regions involved in fearful and anxious behavior. Behav Brain Res 221:118-33. doi: 10.1016/j.bbr.2011.03.008

Adhikari A, Topiwala M a, Gordon J a (2010) Synchronized activity between the ventral hippocampus and the medial prefrontal cortex during anxiety. Neuron 65:257-69. doi: 10.1016/j.neuron.2009.12.002

Adhikari A, Topiwala M a, Gordon J a (2011) Single units in the medial prefrontal cortex with anxiety-related firing patterns are preferentially influenced by ventral hippocampal activity. Neuron 71:898-910. doi: 10.1016/j.neuron.2011.07.027

Akinci MK, Schofield PR (1999) Widespread expression of GABA(A) receptor subunits in peripheral tissues. Neurosci Res 35:145-53.

Ala TA, Hughes LF, Kyrouac GA, et al. (2002) The Mini-Mental State exam may help in the differentiation of dementia with Lewy bodies and Alzheimer's disease. Int J Geriatr Psychiatry 17:503-9. doi: 10.1002/gps.550

Alberini CM (2009) Transcription Factors in Long-Term Memory and Synaptic Plasticity. Physiol Rev 89:121-145. doi: 10.1152/physrev.00017.2008.

Androutsellis-Theotokis a, McCormack WJ, Bradford HF, et al. (1996) The depolarisationinduced release of [125I]BDNF from brain tissue. Brain Res 743:40-8.

Ao H, Ko SW, Zhuo M (2006) CREB activity maintains the survival of cingulate cortical pyramidal neurons in the adult mouse brain. Mol Pain. doi: 10.1186/1744-8069-2-15

Argyropoulos S V, Sandford JJ, Nutt DJ (2001) The psychobiology of anxiolytic drugs Part 2 : pharmacological treatments of anxiety. Pharmacol Ther 88:213-227.

Ariga M, Neitzert B, Nakae S, et al. (2004) Nonredundant function of phosphodiesterases 4D and $4 B$ in neutrophil recruitment to the site of inflammation. J Immunol 173:7531-7538.

Arnsten AFT (2011) Prefrontal cortical network connections: key site of vulnerability in stress and schizophrenia. Int J Dev Neurosci 29:215-23. doi: 10.1016/j.ijdevneu.2011.02.006

Arnsten AFT (2009) Stress signalling pathways that impair prefrontal cortex structure and function. Nat Rev Neurosci 10:410-422. doi: 10.1038/nrn2648.Stress

Arnsten AFT, Jin LE (2012) Guanfacine for the treatment of cognitive disorders: A century of discoveries at Yale. Yale J Biol Med 85:45-58. 
Arnsten AFT, Paspalas CD, Gamo NJ, et al. (2010) Dynamic Network Connectivity: A new form of neuroplasticity. Trends Cogn Sci 14:365-75. doi: 10.1016/j.tics.2010.05.003

Arnsten AFT, Ramos BP, Birnbaum SG, Taylor JR (2005) Protein kinase A as a therapeutic target for memory disorders: rationale and challenges. Trends Mol Med 11:121-8. doi: 10.1016/j.molmed.2005.01.006

Baillie GS (2009) Compartmentalized signalling: spatial regulation of cAMP by the action of compartmentalized phosphodiesterases. FEBS J 276:1790-9. doi: 10.1111/j.17424658.2009.06926.x

Baillie GS, Adams DR, Bhari N, et al. (2007) Mapping binding sites for the PDE4D5 cAMP-specific phosphodiesterase to the $\mathrm{N}$ - and $\mathrm{C}$-domains of beta-arrestin using spot-immobilized peptide arrays. Biochem J 404:71-80. doi: 10.1042/BJ20070005

Baillie GS, Huston E, Scotland G, et al. (2002) TAPAS-1, a novel microdomain within the unique $\mathrm{N}$-terminal region of the PDE4A1 cAMP-specific phosphodiesterase that allows rapid, Ca2+-triggered membrane association with selectivity for interaction with phosphatidic acid. J Biol Chem 277:28298-309. doi: 10.1074/jbc.M108353200

Baillie GS, Sood A, Mcphee I, et al. (2003) Beta-Arrestin-mediated PDE4 cAMP phosphodiesterase recrutiment regulates beta-adrenoceptor switching from Gs to Gi. 100:

Baldwin DS, Ajel K, Masdrakis VG, et al. (2013) Pregabalin for the treatment of generalized anxiety disorder: an update. Neuropsychiatr Dis Treat 9:883-92. doi: 10.2147/NDT.S36453

Bandelow B, Boerner J R, Kasper S, et al. (2013) The diagnosis and treatment of generalized anxiety disorder. Dtsch Arztebl Int 110:300-10. doi: 10.3238/arztebl.2013.0300

Bannerman DM, Grubb M, Deacon RMJ, et al. (2003) Ventral hippocampal lesions affect anxiety but not spatial learning. Behav Brain Res 139:197-213.

Bannerman DM, Yee BK, Good M a, et al. (1999) Double dissociation of function within the hippocampus: a comparison of dorsal, ventral, and complete hippocampal cytotoxic lesions. Behav Neurosci 113:1170-88.

Barad M, Bourtchouladze R, Winder DG, et al. (1998) Rolipram, a type IV-specific phosphodiesterase inhibitor, facilitates the establishment of long-lasting long-term potentiation and improves memory. Proc Natl Acad Sci U S A 95:15020-5.

Barnes NM, Neumaier JF (2011) Neuronal 5-HT Receptors and SERT. Tocris Biosci. Sci. Rev. Ser. 34: 
Beard MB, Huston E, Campbell L, et al. (2002) In addition to the SH3 binding region, multiple regions within the $\mathrm{N}$-terminal noncatalytic portion of the CAMP-specific phosphodiesterase, PDE4A5, contribute to its intracellular targeting. Cell Signal 14:453-65.

Beard MB, O'Connell JC, Bolger GB, Houslay MD (1999) The unique N-terminal domain of the CAMP phosphodiesterase PDE4D4 allows for interaction with specific SH3 domains. FEBS Lett 460:173-7.

Berchtold NC, Cotman CW (1998) Evolution in the conceptualization of dementia and Alzheimer's disease: Greco-Roman period to the 1960s. Neurobiol Aging 19:173-189.

Bertolino A, Crippa D, di Dio S, et al. (1988) Rolipram versus imipramine in inpatients with major, "minor" or atypical depressive disorder: a double-blind double-dummy study aimed at testing a novel therapeutic approach. Int Clin Psychopharmacol 3:245-53.

Bito H, Deisseroth K, Tsien RW (1996) CREB phosphorylation and dephosphorylation: a Ca(2+)and stimulus duration-dependent switch for hippocampal gene expression. Cell 87:120314.

Blackman B, Horner K, Heidmann J, et al. (2011) PDE4D and PDE4B Function in Distinct Subcellular Compartments in Mouse Embryonic Fibroblasts. J Biol Chem 286:12590-12601.

Blundell J, Adamec R (2006) Elevated pCREB in the PAG after exposure to the elevated plus maze in rats previously exposed to a cat. Behav Brain Res 175:285-95. doi: 10.1016/j.bbr.2006.08.029

Bolger G, Michaeli T, Martins T, et al. (1993) A family of human phosphodiesterases homologous to the dunce learning and memory gene product of Drosophila melanogaster are potential targets for antidepressant drugs. Mol Cell Biol 13:6558-71.

Bolger GB, Erdogan S, Jones RE, et al. (1997) Characterization of five different mRNAs from the human cAMP-specific phosphodiesterase PDE4D gene. Biochem J 328:539-548.

Bolger GB, McCahill A, Huston E, et al. (2003a) The unique amino-terminal region of the PDE4D5 cAMP phosphodiesterase isoform confers preferential interaction with betaarrestins. J Biol Chem 278:49230-8. doi: 10.1074/jbc.M303772200

Bolger GB, Mcphee I, Houslay MD (1996) Alternative Splicing of cAMP-specific Phosphodiesterase mRNA Transcripts, Characterization of a A Novel Tissue-specific Isoform,. J Biol Chem 271:1065-1071.

Bolger GB, Peden AH, Steele MR, et al. (2003b) Attenuation of the activity of the cAMP-specific phosphodiesterase PDE4A5 by interaction with the immunophilin XAP2. J Biol Chem 278:33351-63. doi: 10.1074/jbc.M303269200 
Borlikova G, Endo S (2009) Inducible cAMP early repressor (ICER) and brain functions. Mol Neurobiol 40:73-86. doi: 10.1007/s12035-009-8072-1

Bourdeau I, Stratakis CA (2002) Cyclic AMP-dependent signaling aberrations in macronodular adrenal disease. Ann N Y Acad Sci 968:240-55.

Bourtchuladze R, Frenguelli B, Blendy J, et al. (1994) Deficient long-term memory in mice with a targeted mutation of the cAMP-responsive element-binding protein. Cell 79:59-68.

Brake WG, Flores G, Francis D, et al. (2000) Enhanced nucleus accumbens dopamine and plasma corticosterone stress responses in adult rats with neonatal excitotoxic lesions to the medial prefrontal cortex. Neuroscience 96:687-95.

Brody $\mathrm{H}$ (1955) Organization of the cerebral cortex. III. A study of aging in the human cerebral cortex. J Comp Neurol 102:511-6.

Broman-Fulks JJ, Berman ME, Rabian B a, Webster MJ (2004) Effects of aerobic exercise on anxiety sensitivity. Behav Res Ther 42:125-36. doi: 10.1016/S0005-7967(03)00103-7

Bruno O, Fedele E, Prickaerts J, et al. (2011) GEBR-7b, a novel PDE4D selective inhibitor that improves memory in rodents at non-emetic doses. Br J Pharmacol 164:2054-63. doi: 10.1111/j.1476-5381.2011.01524.x

Bruss MD, Richter W, Horner K, et al. (2008) Critical role of PDE4D in beta2-adrenoceptordependent CAMP signaling in mouse embryonic fibroblasts. J Biol Chem 283:22430-42. doi: 10.1074/jbc.M803306200

Buras A, Battle L, Landers E, et al. (2014) Thyroid hormones regulate anxiety in the male mouse. Horm Behav 65:88-96. doi: 10.1016/j.yhbeh.2013.11.008

Burgin AB, Magnusson OT, Singh J, et al. (2010) Design of phosphodiesterase 4D (PDE4D) allosteric modulators for enhancing cognition with improved safety. Nat Biotechnol 28:6370. doi: $10.1038 / \mathrm{nbt} .1598$

Busner J, Targum S (2007) Global Impressions Scale : Applying a Research. Psychiatry July:2837.

Byerly MS, Simon J, Lebihan-Duval E, et al. (2009) Effects of BDNF, T3, and corticosterone on expression of the hypothalamic obesity gene network in vivo and in vitro. Am J Physiol Regul Integr Comp Physiol 296:R1180-9. doi: 10.1152/ajpregu.90813.2008

Campfield L a. (1998) Strategies and Potential Molecular Targets for Obesity Treatment. Science (80- ) 280:1383-1387. doi: 10.1126/science.280.5368.1383 
Carlezon W a, Duman RS, Nestler EJ (2005) The many faces of CREB. Trends Neurosci 28:43645. doi: 10.1016/j.tins.2005.06.005

Celikyurt IK, Ulak G, Mutlu O, et al. (2012) Positive impact of levetiracetam on emotional learning and memory in naive mice. Life Sci 90:185-9. doi: 10.1016/j.Ifs.2011.11.003

Cha-molstad H, Keller DM, Yochum GS, et al. (2004) Cell-type-specific binding of the transcription factor CREB to the cAMP-response element. Proc Natl Acad Sci USA 101:13572-7.

Chandrasekaran A, Toh KY, Low SH, et al. (2008) Identification and characterization of novel mouse PDE4D isoforms: molecular cloning, subcellular distribution and detection of isoform-specific intracellular localization signals. Cell Signal 20:139-53. doi: 10.1016/j.cellsig.2007.10.003

Cherry J a, Pho V (2002) Characterization of cAMP degradation by phosphodiesterases in the accessory olfactory system. Chem Senses 27:643-52.

Cherry JA, Davis RL (1999) Cyclic AMP Phosphodiesterases are Localized in Regions of the Mouse Brain Associated With Reinforcement , Movement, and Affect. J Comp Neurol 301:287-301.

Chessick CA, Allen MH, Thase M, et al. (2006) Azapirones for generalized anxiety disorder. Cochrane database Syst Rev CD006115. doi: 10.1002/14651858.CD006115

Cheung Y, Kan Z, Garrett-engele P, et al. (2007) PDE4B5 , a Novel , Super-Short , Brain-Specific cAMP Phosphodiesterase-4 Variant Whose Isoform-Specifying N-Terminal Region Is Identical to That of cAMP. J Pharmacol Exp Ther 322:600-609. doi: 10.1124/jpet.107.122218.The

Cho CH, Cho DH, Seo MR, Juhnn YS (2000) Differential changes in the expression of cyclic nucleotide phosphodiesterase isoforms in rat brains by chronic treatment with electroconvulsive shock. Exp Mol Med 32:110-4. doi: 10.1038/emm.2000.19

Colman E, Golden J, Roberts M, et al. (2012) The FDA's assessment of Two Drugs for Chronic Weight Management. New Englang J Med 367:2012-2014.

Conca A, König P, Hausmann A (2000) Transcranial magnetic stimulation induces "pseudoabsence seizure". Acta Psychiatr Scand 101:246-8; discussion 248-9.

Cone J, Wang S, Tandon N, et al. (1999) Comparison of the effects of cilostazol and milrinone on intracellular cAMP levels and cellular function in platelets and cardiac cells. J Cardiovasc Pharmacol 34:497-504. 
Conkright MD, Guzman E, Flechner L, et al. (2003) Genome-Wide Analysis of CREB Target Genes Reveals A Core Promoter Requirement for cAMP Responsiveness Salk Institute for Biological Studies. Mol Cell 11:1101-1108.

Conti M, Richter W, Mehats C, et al. (2003a) Cyclic AMP-specific PDE4 phosphodiesterases as critical components of cyclic AMP signaling. J Biol Chem 278:5493-6. doi: 10.1074/jbc.R200029200

Conti M, Richter W, Mehats C, et al. (2003b) Cyclic AMP-specific PDE4 phosphodiesterases as critical components of cyclic AMP signaling. J Biol Chem 278:5493-6. doi: 10.1074/jbc.R200029200

Correa-Sales C, Rabin BC, Maze M (1992) A hypnotic response to dexmedetomidine, an alpha 2 agonist, is mediated in the locus coeruleus in rats. Anesthesiology 76:948-52.

Crismon ML (1994) Tacrine: first drug approved for Alzheimer's disease. Ann Pharmacother 28:744-51.

Cruz L, Roe DL, Urbanc B, et al. (2004) Age-related reduction in microcolumnar structure in area 46 of the rhesus monkey correlates with behavioral decline. Proc Natl Acad Sci U S A 101:15846-51. doi: 10.1073/pnas.0407002101

Cygnar KD, Zhao H (2009) Phosphodiesterase 1C is dispensable for rapid response termination of olfactory sensory neurons. Nat Neurosci 12:454-62. doi: 10.1038/nn.2289

D'Sa C, Eisch AJ, Bolger GB, Duman RS (2005) Differential expression and regulation of the cAMP-selective phosphodiesterase type $4 \mathrm{~A}$ splice variants in rat brain by chronic antidepressant administration. Eur J Neurosci 22:1463-75. doi: 10.1111/j.14609568.2005.04321.x

Danysz W, Parsons CG, MÖbius H-Jö, et al. (2000) Neuroprotective and symptomatological action of memantine relevant for alzheimer's disease - a unified glutamatergic hypothesis on the mechanism of action. Neurotox Res 2:85-97. doi: 10.1007/BF03033787

Dash P, Hochner B, Kandel E (1990) Injection of the cAMP-responsive element into the nucleus of Aplysia sensory neurons blocks long-term facilitation. Nature 345::718-21.

Davidson JR (2009) First-line pharmacotherapy approaches for generalized anxiety disorder. J Clin Psychiatry 70 Suppl 2:25-31.

Davis RL, Takayasu H, Eberwine M, Myres J (1989) Cloning and characterization of mammalian homologs of the Drosophila dunce+ gene. Proc Natl Acad Sci U S A 86:3604-8.

Deacon BJ, Abramowitz JS (2004) Cognitive and behavioral treatments for anxiety disorders: a review of meta-analytic findings. J Clin Psychol 60:429-41. doi: 10.1002/jclp.10255 
Deacon RMJ, Catherine P, Rawlins JNP (2003) Effects of medial prefrontal cortex cytotoxic lesions in mice. Behav Brain Res 139:139-55.

Degroot A, Treit D (2004) Anxiety is functionally segregated within the septo-hippocampal system. Brain Res 1001:60-71. doi: 10.1016/j.brainres.2003.10.065

Dodge KL, Khouangsathiene S, Kapiloff MS, et al. (2001) mAKAP assembles a protein kinase A/PDE4 phosphodiesterase CAMP signaling module. EMBO J 20:1921-30. doi: 10.1093/emboj/20.8.1921

Dodge-Kafka KL, Soughayer J, Pare GC, et al. (2005) The protein kinase A anchoring protein mAKAP coordinates two integrated cAMP effector pathways. Nature 437:574-8. doi: 10.1038/nature03966

Doseyici S, Mehmetoglu I, Toker A, et al. (2014) The effects of forskolin and rolipram on cAMP, cGMP and free fatty acid levels in diet induced obesity. Biotech Histochem. doi: $10.3109 / 10520295.2014 .883463$

Drachman D a (2006) Aging of the brain, entropy, and Alzheimer disease. Neurology 67:134052. doi: 10.1212/01.wnl.0000240127.89601.83

Dyke HJ, Montana JG (2002) Update on the therapeutic potential of PDE4 inhibitors. Expert Opin Investig Drugs 11:1-13. doi: 10.1517/13543784.11.1.1

Eckel RH (2008) Nonsurgical Management of Obesity in Adults. New Englang J Med 358:19411950.

Egawa T, Mishima K, Matsumoto Y, et al. (1997) Rolipram and its optical isomers, phosphodiesterase 4 inhibitors, attenuated the scopolamine-induced impairments of learning and memory in rats. Jpn J Pharmacol 75:275-81.

Eich H, Agelink MW, Lehmann E, et al. (2000) Acupuncture in patients with minor depressive episodes and generalized anxiety. Results of an experimental study. Fortschr Neurol Psychiatr 68:137-44. doi: 10.1055/s-2000-11624

Engels P, Abdel'AI S, Hulley P, Lübbert H (1995) Brain distribution of four rat homologues of the Drosophila dunce cAMP phosphodiesterase. J Neurosci Res 41:169-78. doi: 10.1002/jnr.490410204

Ennaceur a (2010) One-trial object recognition in rats and mice: methodological and theoretical issues. Behav Brain Res 215:244-54. doi: 10.1016/j.bbr.2009.12.036

Faber J, Sørensen TI, Lumholtz IB, et al. (1981) Serum levels of T4, T3, reverse T3, 3,3' diiodothyronine and 3',5'-diiodothyronine in obesity, before and after jejuno-ileal bypass. Clin Endocrinol (Oxf) 14:119-24. 
Fatemi SH, King DP, Reutiman TJ, et al. (2008) PDE4B polymorphisms and decreased PDE4B expression are associated with schizophrenia. Schizophr Res 101:36-49. doi: 10.1016/j.schres.2008.01.029

Figueiredo HF, Bruestle A, Bodie B, et al. (2003) The medial prefrontal cortex differentially regulates stress-induced c-fos expression in the forebrain depending on type of stressor. Eur J Neurosci 18:2357-2364. doi: 10.1046/j.1460-9568.2003.02932.x

Figurov A, Pozzo-Miller LD, Olafsson P, et al. (1996) Regulation of synaptic responses to highfrequency stimulation and LTP by neurotrophins in the hippocampus. Nature 381:706-9. doi: $10.1038 / 381706 a 0$

Finkbeiner S, Tavazoie SF, Maloratsky A, et al. (1997) CREB: a major mediator of neuronal neurotrophin responses. Neuron 19:1031-47.

Fisher BL, Schauer P (2002) Medical and surgical options in the treatment of severe obesity. Am J Surg 184:9S-16S.

Forkmann T, Scherer A, Boecker M, et al. (2011) The Clinical Global Impression Scale and the influence of patient or staff perspective on outcome. BMC Psychiatry 11:83. doi: $10.1186 / 1471-244 X-11-83$

Fox D, Burgin AB, Gurney ME (2014) Structural basis for the design of selective phosphodiesterase 4B inhibitors. Cell Signal 26:657-63. doi: 10.1016/j.cellsig.2013.12.003

Fujita M, Hines CS, Zoghbi SS, et al. (2012) Downregulation of brain phosphodiesterase type IV measured with 11C-(R)-rolipram positron emission tomography in major depressive disorder. Biol Psychiatry 72:548-54. doi: 10.1016/j.biopsych.2012.04.030

Gadde K, Franciscy D, Wagner HR, Krishnan KRR (2014) Zonisamide for Weight Loss in Obese Adults A Randomized Controlled Trial. JAMA 289:

Gamo NJ, Arnsten AFT (2011) Molecular modulation of prefrontal cortex: rational development of treatments for psychiatric disorders. Behav Neurosci 125:282-96. doi: 10.1037/a0023165

Garcia R, Vouimba RM, Baudry M, Thompson RF (1999) The amygdala modulates prefrontal cortex activity relative to conditioned fear. Nature 402:294-6. doi: 10.1038/46286

Geinisman $Y$ (1999) Age-related decline in memory function: is it associated with a loss of synapses? Neurobiol Aging 20:353-6; discussion 359-60.

Geinisman Y, Ganeshina O, Yoshida R, et al. (2004) Aging, spatial learning, and total synapse number in the rat CA1 stratum radiatum. Neurobiol Aging 25:407-16. doi:

10.1016/j.neurobiolaging.2003.12.001 
George MS, Nahas Z, Borckardt JJ, et al. (2007) Vagus nerve stimulation for the treatment of depression and other neuropsychiatric disorders. Expert Rev Neurother 7:63-74. doi: 10.1586/14737175.7.1.63

Gerits N, Kostenko S, Shiryaev A, et al. (2008) Relations between the mitogen-activated protein kinase and the CAMP-dependent protein kinase pathways: comradeship and hostility. Cell Signal 20:1592-607. doi: 10.1016/j.cellsig.2008.02.022

Giannaris EL, Rosene DL (2012) A stereological study of the numbers of neurons and glia in the primary visual cortex across the lifespan of male and female rhesus monkeys. J Comp Neurol 520:3492-508. doi: 10.1002/cne.23101

Giorgi M, Modica A, Pompili A, et al. (2004) The induction of cyclic nucleotide phosphodiesterase 4 gene (PDE4D) impairs memory in a water maze task. Behav Brain Res 154:99-106. doi: 10.1016/j.bbr.2004.01.024

Glisky E (2007) Changes in Cognitive Function in Human Aging. Brain Aging Model. Methods, Mech. Boca Rat. CRC Press Chapter 1:

Gonzalez LE, Rujano M, Tucci S, et al. (2000) Medial prefrontal transection enhances social interaction. I: behavioral studies. Brain Res 887:7-15.

Gordon J a, Lacefield CO, Kentros CG, Hen R (2005) State-dependent alterations in hippocampal oscillations in serotonin $1 \mathrm{~A}$ receptor-deficient mice. J Neurosci 25:6509-19. doi: 10.1523/JNEUROSCI.1211-05.2005

Graham DP, Cully JA, Snow AL, et al. The Alzheimer's Disease Assessment Scale-Cognitive subscale: normative data for older adult controls. Alzheimer Dis Assoc Disord 18:236-40.

Greenberg P, Sisitsky T, Kessler R, et al. (1999) The economic burden of anxiety disorders in the 1990s. J Clin Psychiatry 60:427-435.

Greene M, Thackeray JT, Kenk M, et al. (2009) Reduced in vivo phosphodiesterase-4 response to acute noradrenaline challenge in diet-induced obese rats. 202:196-202. doi: 10.1139/Y09-001

Guiot-Goffioul F, Gerard-Vandenhove MA, Troisfontaines B, et al. (1987) [Preliminary results of a double-blind study between rolipram and desipramine in hospitalized patients with major depressive symptoms]. Acta Psychiatr Belg 87:230-5.

Gunter RW, Whittal ML (2010) Dissemination of cognitive-behavioral treatments for anxiety disorders: Overcoming barriers and improving patient access. Clin Psychol Rev 30:194-202. doi: 10.1016/j.cpr.2009.11.001

Guy W (1976) Assessment Manual for Psychopharmacology. 1: 
Hains AB, Arnsten AFT (2008) Molecular mechanisms of stress-induced prefrontal cortical impairment: implications for mental illness. Learn Mem 15:551-64. doi: 10.1101/Im.921708

Hains AB, Vu MAT, Maciejewski PK, et al. (2009) Inhibition of protein kinase $C$ signaling protects prefrontal cortex dendritic spines and cognition from the effects of chronic stress. Proc Natl Acad Sci U S A 106:17957-62. doi: 10.1073/pnas.0908563106

Hall J, Thomas KL, Everitt BJ (2000) Rapid and selective induction of BDNF expression in the hippocampus during contextual learning. Nat Neurosci 3:533-5. doi: 10.1038/75698

Hamilton M (1967) Development of a rating scale for primary depressive illness. Br J Soc Clin Psychol 6:278-96.

Hamilton M (1959) THE ASSESSMENT OF ANXIETY STATES BY RATING. Br J Med Psychol 32:5055. doi: 10.1111/j.2044-8341.1959.tb00467.x

Hanoune J, Defer N (2001) Regulation and role of adenylyl cyclase isoforms. Annu Rev Pharmacol Toxicol 41:145-74. doi: 10.1146/annurev.pharmtox.41.1.145

Hansen RT, Conti M, Zhang H-T (2014) Mice deficient in phosphodiesterase-4A display anxiogenic-like behavior. Psychopharmacology (Berl). doi: 10.1007/s00213-014-3480-y

Hansen RT, Zhang H-T (2013) Senescent-induced dysregulation of CAMP/CREB signaling and correlations with cognitive decline. Brain Res 1516:93-109. doi:

10.1016/j.brainres.2013.04.033

Haug $H$ (1985) Are neurons of the human cerebral cortex really lost during aging? A morphometric examination. Adv Appl Neurol Sci 2:150-163.

He Y, Yang DZ, Yu H, et al. (2013) Genetic variants of phosphodiesterase 4D gene are associated with an enhanced risk for ischemic stroke in young Chinese population. Neurol India 61:21-5. doi: 10.4103/0028-3886.108131

Heaslip RJ, Evans DY (1995) Emetic, central nervous system, and pulmonary activities of rolipram in the dog. Eur J Pharmacol 286:281-90.

Hebenstreit GF, Fellerer K, Fichte K, et al. (1989) Rolipram in major depressive disorder: results of a double-blind comparative study with imipramine. Pharmacopsychiatry 22:156-60. doi: 10.1055/s-2007-1014599

Heidbreder C a, Groenewegen HJ (2003) The medial prefrontal cortex in the rat: evidence for a dorso-ventral distinction based upon functional and anatomical characteristics. Neurosci Biobehav Rev 27:555-579. doi: 10.1016/j.neubiorev.2003.09.003 
Heisler LK, Chu H-M, Brennan TJ, et al. (1998) Elevated anxiety and antidepressant-like responses in serotonin 5-HT 1A receptor mutant mice. Proc Natl Acad Sci U S A 95:1504915054.

Helmreich DL, Parfitt DB, Lu X-Y, et al. (2005) Relation between the hypothalamic-pituitarythyroid (HPT) axis and the hypothalamic-pituitary-adrenal (HPA) axis during repeated stress. Neuroendocrinology 81:183-92. doi: 10.1159/000087001

Helmreich DL, Tylee D (2011) Thyroid hormone regulation by stress and behavioral differences in adult male rats. Horm Behav 60:284-91. doi: 10.1016/j.yhbeh.2011.06.003

Henderson G, Tomlinson B, Gibson P (1980) Cell counts in human cerebral cortex in normal adults throughout life, using an image analysis computer. J Neurol Sci 46:113-36.

Herrup K (2010) Reimagining Alzheimer's disease--an age-based hypothesis. J Neurosci 30:16755-62. doi: 10.1523/JNEUROSCI.4521-10.2010

Heyer EJ, Mergeche JL, Ward JT, et al. (2013) Phosphodiesterase 4D single-nucleotide polymorphism 83 and cognitive dysfunction in carotid endarterectomy patients. Neurosurgery 73:791-6; discussion 796. doi: 10.1227/NEU.0000000000000085

Hiester BG, Galati DF, Salinas PC, Jones KR (2013) Neurotrophin and Wnt signaling cooperatively regulate dendritic spine formation. Mol Cell Neurosci 56C:115-127. doi: 10.1016/j.mcn.2013.04.006

Ho D, Yan L, Zhao X, et al. (2011) Inhibition of Adenylyl Cyclase Type 5 Protects Against Obesity and Diabetes. Circulation

Holmes A, Wellman CL (2009) Stress-induced prefrontal reorganization and executive dysfunction in rodents. Neurosci Biobehav Rev 33:773-83. doi: 10.1016/j.neubiorev.2008.11.005

Horton YM, Sullivan M, Houslay MD (1995) Molecular cloning of a novel splice variant of human type IVA (PDE-IVA) cyclic AMP phosphodiesterase and localization of the gene to the p13.2-q12 region of human chromosome 19 [corrected]. Biochem J 308 ( Pt 2:683-91.

Houslay MD (2010) Underpinning compartmentalised cAMP signalling through targeted cAMP breakdown. Trends Biochem Sci 35:91-100. doi: 10.1016/j.tibs.2009.09.007

Houslay MD, Adams DR (2003) PDE4 cAMP phosphodiesterases: modular enzymes that orchestrate signalling cross-talk, desensitization and compartmentalization. Biochem J 370:1-18. doi: 10.1042/BJ20021698 
Houslay MD, Baillie GS, Maurice DH (2007) cAMP-Specific phosphodiesterase-4 enzymes in the cardiovascular system: a molecular toolbox for generating compartmentalized cAMP signaling. Circ Res 100:950-66. doi: 10.1161/01.RES.0000261934.56938.38

Hu W, Lu T, Chen A, et al. (2011) Inhibition of phosphodiesterase-4 decreases ethanol intake in mice. Psychopharmacology (Berl). doi: 10.1007/s00213-011-2290-8

Huston E, Beard M, McCallum F, et al. (2000) The cAMP-specific phosphodiesterase PDE4A5 is cleaved downstream of its $\mathrm{SH} 3$ interaction domain by caspase-3. Consequences for altered intracellular distribution. J Biol Chem 275:28063-74. doi: 10.1074/jbc.M906144199

Huston E, Lumb S, Russell a, et al. (1997) Molecular cloning and transient expression in COS7 cells of a novel human PDE4B CAMP-specific phosphodiesterase, HSPDE4B3. Biochem J 328 ( Pt 2:549-58.

Hynd MR, Scott HL, Dodd PR (2004) Glutamate-mediated excitotoxicity and neurodegeneration in Alzheimer's disease. Neurochem Int 45:583-95. doi: 10.1016/j.neuint.2004.03.007

Imaizumi M, Miyazaki S, Onodera K (1994) Effects of a non-xanthine adenosine antagonist, CGS 15943, and a phosphodiesterase inhibitor, Ro 20-1724, in a light/dark test in mice. Methods Find Exp Clin Pharmacol 16:717-21.

Imanishi T, Sawa A, Ichimaru Y, et al. (1997) Ameliorating effects of rolipram on experimentally induced impairments of learning and memory in rodents. Eur J Pharmacol 321:273-8.

Impey S, Mark M, Villacres E, et al. (1996) Induction of CRE-mediated gene expression by stimuli that generate long-lasting LTP in area CA1 of the hippocampus. Neuron 16:973-82.

loannides-Demos LL, Piccenna L, McNeil JJ (2011) Pharmacotherapies for obesity: past, current, and future therapies. J Obes 2011:179674. doi: 10.1155/2011/179674

Jack CR, Petersen RC, Xu YC, et al. (1997) Medial temporal atrophy on MRI in normal aging and very mild Alzheimer's disease. Neurology 49:786-794. doi: 10.1212/WNL.49.3.786

Jang D-J, Park S-W, Lee J-A, et al. (2010) N termini of apPDE4 isoforms are responsible for targeting the isoforms to different cellular membranes. Learn Mem 17:469-79. doi: $10.1101 / \mathrm{Im} .1899410$

Jeanneteau FD, Lambert WM, Ismaili N, et al. (2012) BDNF and glucocorticoids regulate corticotrophin-releasing hormone $(\mathrm{CRH})$ homeostasis in the hypothalamus. Proc Natl Acad Sci U S A 109:1305-10. doi: 10.1073/pnas.1114122109

Jefferson AL, Cosentino SA, Ball SK, et al. (2002) Errors produced on the mini-mental state examination and neuropsychological test performance in Alzheimer's disease, ischemic vascular dementia, and Parkinson's disease. J Neuropsychiatry Clin Neurosci 14:311-20. 
Jin S-LC, Conti M (2002) Induction of the cyclic nucleotide phosphodiesterase PDE4B is essential for LPS-activated TNF-alpha responses. Proc Natl Acad Sci U S A 99:7628-33. doi: 10.1073/pnas.122041599

Jin S-LC, Goya S, Nakae S, et al. (2010) Phosphodiesterase 4B is essential for T(H)2-cell function and development of airway hyperresponsiveness in allergic asthma. J Allergy Clin Immunol 126:1252-9.e12. doi: 10.1016/j.jaci.2010.08.014

Jin S-LC, Lan L, Zoudilova M, Conti M (2005a) Specific role of phosphodiesterase 4B in lipopolysaccharide-induced signaling in mouse macrophages. J Immunol 175:1523-31.

Jin S-LC, Latour AM, Conti M (2005b) Generation of PDE4 knockout mice by gene targeting. Methods Mol Biol 307:191-210. doi: 10.1385/1-59259-839-0:191

Jin S-LC, Richard F, Kuo W-P, et al. (1999) Impaired growth and fertility of cAMP-specific phosphodiesterase PDE4D-deficient mice. Proc Natl Acad Sci U S A 96:11998-2003.

Johannessen M, Delghandi MP, Moens U (2004) What turns CREB on? Cell Signal 16:1211-27. doi: 10.1016/j.cellsig.2004.05.001

Johansson EM, Reyes-Irisarri E, Mengod G (2012) Comparison of cAMP-specific phosphodiesterase mRNAs distribution in mouse and rat brain. Neurosci Lett 525:1-6. doi: 10.1016/j.neulet.2012.07.050

Johnson KR, Nicodemus-Johnson J, Danziger RS (2010) An evolutionary analysis of cAMPspecific Phosphodiesterase 4 alternative splicing. BMC Evol Biol 10:247. doi: 10.1186/14712148-10-247

Johnston LA, Erdogan S, Cheung YF, et al. (2004) Expression, intracellular distribution and basis for lack of catalytic activity of the PDE4A7 isoform encoded by the human PDE4A CAMPspecific phosphodiesterase gene. Biochem J 380:371-84. doi: 10.1042/BJ20031662

Kähler AK, Otnaess MK, Wirgenes K V, et al. (2010) Association study of PDE4B gene variants in Scandinavian schizophrenia and bipolar disorder multicenter case-control samples. Am J Med Genet B Neuropsychiatr Genet 153B:86-96. doi: 10.1002/ajmg.b.30958

Kaneko M, Xie Y, An JJ, et al. (2012) Dendritic BDNF synthesis is required for late-phase spine maturation and recovery of cortical responses following sensory deprivation. J Neurosci 32:4790-802. doi: 10.1523/JNEUROSCI.4462-11.2012

Katzman M a (2009) Current considerations in the treatment of generalized anxiety disorder. CNS Drugs 23:103-20.

Kauvar LM (1982) DEFECTIVE CYCLIC ADENOSINE 3':5-MONOPHOSPHATE PHOSPHODIESTERASE IN THE DROSOPHILA MEMORY MUTANT DUNCE. J Neurosci 2:1347-1358. 
Kelly MP, Adamowicz W, Bove S, et al. (2014) Select 3',5'-cyclic nucleotide phosphodiesterases exhibit altered expression in the aged rodent brain. Cell Signal 26:383-97. doi:

10.1016/j.cellsig.2013.10.007

Kessler R, Chiu WT, Demler O, Walkters E (2010) Prevalence, Severity, and Comorbidity of Twelve-month DSM-IV Disorders in the National Comorbidity Survey Replication (NCS-R). Arch Gen Psychiatry 62:617-627. doi: 10.1001/archpsyc.62.6.617.Prevalence

Khan A, Faucett J, Lichtenberg P, et al. (2012) A systematic review of comparative efficacy of treatments and controls for depression. PLoS One 7:e41778. doi: 10.1371/journal.pone.0041778

Kirkwood G, Rampes H, Tuffrey V, et al. (2005) Yoga for anxiety: a systematic review of the research evidence. Br J Sports Med 39:884-91; discussion 891. doi: 10.1136/bjsm.2005.018069

Klonoff DC, Greenway F (2008) Drugs in the Pipeline for the Obesity Market. J Diabetes Sci Technol 2:913-918. doi: 10.1177/193229680800200525

Kojima M, Takei N, Numakawa T, et al. (2001) Biological characterization and optical imaging of brain-derived neurotrophic factor-green fluorescent protein suggest an activity-dependent local release of brain-derived neurotrophic factor in neurites of cultured hippocampal neurons. J Neurosci Res 64:1-10.

Kolosionek E, Savai R, Ghofrani HA, et al. (2009) Expression and Activity of Phosphodiesterase Isoforms during Epithelial Mesenchymal Transition : The Role of Phosphodiesterase 4. Mol Biol Cell 20:4751-4765. doi: 10.1091/mbc.E09

Korte M, Kang H, Bonhoeffer T, Schuman E (1998) A role for BDNF in the late-phase of hippocampal long-term potentiation. Neuropharmacology 37:553-9.

Korte M, Staiger V, Griesbeck O, et al. (1996) The involvement of brain-derived neurotrophic factor in hippocampal long-term potentiation revealed by gene targeting experiments. J Physiol Paris 90:157-64.

Korte SM (2001) Corticosteroids in relation to fear, anxiety and psychopathology. Neurosci Biobehav Rev 25:117-42.

Kurien BT, Scofield RH (1999) Mouse urine collection using clear plastic wrap. Lab Anim 33:8386. doi: $10.1258 / 002367799780578525$

Lacroix L, Broersen LM, Weiner I, Feldon J (1998) The effects of excitotoxic lesion of the medial prefrontal cortex on latent inhibition, prepulse inhibition, food hoarding, elevated plus maze, active avoidance and locomotor activity in the rat. Neuroscience 84:431-42. 
Lakics V, Karran EH, Boess FG (2010) Quantitative comparison of phosphodiesterase mRNA distribution in human brain and peripheral tissues. Neuropharmacology 59:367-74. doi: 10.1016/j.neuropharm.2010.05.004

Lakshminarasimhan $\mathrm{H}$, Chattarji S (2012) Stress leads to contrasting effects on the levels of brain derived neurotrophic factor in the hippocampus and amygdala. PLoS One 7:e30481. doi: 10.1371/journal.pone.0030481

Lamontagne S, Meadows E, Luk P, et al. (2001) Localization of phosphodiesterase-4 isoforms in the medulla and nodose ganglion of the squirrel monkey. Brain Res 920:84-96.

Laux G, Becker T, Kühne G, et al. (1988) Clinical and biochemical effects of the selective phosphodiesterase inhibitor rolipram in depressed inpatients controlled by determination of plasma level. Pharmacopsychiatry 21:378-9. doi: 10.1055/s-2007-1017016

Li X, Baillie GS, Houslay MD (2009a) Mdm2 directs the ubiquitination of beta-arrestinsequestered cAMP phosphodiesterase-4D5. J Biol Chem 284:16170-82. doi: 10.1074/jbc.M109.008078

Li Y-F, Cheng Y-F, Huang Y, et al. (2011) Phosphodiesterase-4D Knock-Out and RNA Interference-Mediated Knock-Down Enhance Memory and Increase Hippocampal Neurogenesis via Increased cAMP Signaling. J Neurosci 31:172-183. doi: 10.1523/JNEUROSCI.5236-10.2011

Li Y-F, Huang Y, Amsdell SL, et al. (2009b) Antidepressant- and anxiolytic-like effects of the phosphodiesterase-4 inhibitor rolipram on behavior depend on cyclic AMP response element binding protein-mediated neurogenesis in the hippocampus. Neuropsychopharmacology 34:2404-19. doi: 10.1038/npp.2009.66

Lieberman J (2003) History of the Use of Antidepressants in Primary Care. Prim. Care Companion J Clin Psychiatry 5:

Light L (1992) The organization of memory in old age. Craik FIM, Salthouse TA, Ed Handb Aging Cogn Erlbaum; Hillsdale, NJ 111.

Lindstrand A, Grigelioniene G, Nilsson D, et al. (2014) Different mutations in PDE4D associated with developmental disorders with mirror phenotypes. J Med Genet 51:45-54. doi: 10.1136/jmedgenet-2013-101937

Lisanby S (2007) Electroconvulsive Therapy for Depression. New Englang J Med 1939-1945.

Liu K, Wang J, Yu Z, et al. (2013a) [Association study between PDE4D gene polymorphism and ischemic stroke]. Beijing Da Xue Xue Bao 45:359-63. 
Liu X, Zhu R, Li L, et al. (2013b) Genetic polymorphism in PDE4D gene and risk of ischemic stroke in Chinese population: a meta-analysis. PLoS One 8:e66374. doi: 10.1371/journal.pone.0066374

Lleó A, Greenberg SM, Growdon JH (2006) Current pharmacotherapy for Alzheimer's disease. Annu Rev Med 57:513-33. doi: 10.1146/annurev.med.57.121304.131442

Lonze BE, Ginty DD (2002) Function and Regulation of CREB Family Transcription Factors in the Nervous System CREB and its close relatives are now widely accepted. Neuron 35:605-623.

Lorenz RA, Jackson CW, Saitz M (2010) Adjunctive use of atypical antipsychotics for treatmentresistant generalized anxiety disorder. Pharmacotherapy 30:942-51. doi: 10.1592/phco.30.9.942

Lynch MJ, Baillie GS, Houslay MD (2007) cAMP-specific phosphodiesterase-4D5 (PDE4D5) provides a paradigm for understanding the unique non-redundant roles that PDE4 isoforms play in shaping compartmentalized cAMP cell signalling. Biochem Soc Trans 35:938-41. doi: 10.1042/BST0350938

Lynch MJ, Baillie GS, Mohamed A, et al. (2005) RNA silencing identifies PDE4D5 as the functionally relevant cAMP phosphodiesterase interacting with beta arrestin to control the protein kinase A/AKAP79-mediated switching of the beta2-adrenergic receptor to activation of ERK in HEK293B2 cells. J Biol Chem 280:33178-89. doi: 10.1074/jbc.M414316200

Lynex CN, Li Z, Chen ML, et al. (2008) Identification and molecular characterization of a novel PDE4D11 cAMP-specific phosphodiesterase isoform. Cell Signal 20:2247-55. doi: 10.1016/j.cellsig.2008.08.020

Mackenzie KF, Topping EC, Bugaj-Gaweda B, et al. (2008) Human PDE4A8, a novel brainexpressed PDE4 CAMP-specific phosphodiesterase that has undergone rapid evolutionary change. Biochem J 411:361-9. doi: 10.1042/BJ20071251

MacKenzie KF, Wallace D a, Hill E V, et al. (2011) Phosphorylation of cAMP-specific PDE4A5 (phosphodiesterase-4A5) by MK2 (MAPKAPK2) attenuates its activation through protein kinase A phosphorylation. Biochem J 435:755-69. doi: 10.1042/BJ20101184

MacKenzie SJ, Yarwood SJ, Peden a H, et al. (1998) Stimulation of p70S6 kinase via a growth hormone-controlled phosphatidylinositol 3-kinase pathway leads to the activation of a PDE4A cyclic AMP-specific phosphodiesterase in 3T3-F442A preadipocytes. Proc Natl Acad Sci U S A 95:3549-54.

Magni LR, Purgato M, Gastaldon C, et al. (2013) Fluoxetine versus other types of pharmacotherapy for depression. Cochrane database Syst Rev 7:CD004185. 
Marchmont RJ, Houslay MD (1980) A peripheral and an intrinsic enzyme constitute the cyclic AMP phosphodiesterase activity of rat liver plasma membranes. Biochem J 187:381-92.

Maurice DH, Ke H, Ahmad F, et al. (2014) Advances in targeting cyclic nucleotide phosphodiesterases. Nat Rev Drug Discov 13:290-314. doi: 10.1038/nrd4228

McGaugh JL (2004) The amygdala modulates the consolidation of memories of emotionally arousing experiences. Annu Rev Neurosci 27:1-28. doi:

10.1146/annurev.neuro.27.070203.144157

McHugh SB, Deacon RMJ, Rawlins JNP, Bannerman DM (2004) Amygdala and ventral hippocampus contribute differentially to mechanisms of fear and anxiety. Behav Neurosci 118:63-78. doi: 10.1037/0735-7044.118.1.63

McPhee I, Cochran S, Houslay MD (2001) The novel long PDE4A10 cyclic AMP phosphodiesterase shows a pattern of expression within brain that is distinct from the long PDE4A5 and short PDE4A1 isoforms. Cell Signal 13:911-8.

McPhee I, Pooley L, Lobban M, et al. (1995) Identification, characterization and regional distribution in brain of RPDE-6 (RNPDE4A5), a novel splice variant of the PDE4A cyclic AMP phosphodiesterase family. Biochem J 310 ( Pt 3:965-74.

Menniti FS, Faraci WS, Schmidt CJ (2006) Phosphodiesterases in the CNS: targets for drug development. Nat Rev Drug Discov 5:660-70. doi: 10.1038/nrd2058

Millar JK, Pickard BS, Mackie S, et al. (2005) DISC1 and PDE4B are interacting genetic factors in schizophrenia that regulate cAMP signaling. Science 310:1187-91. doi:

10.1126/science.1112915

Miró X, Pérez-Torres S, Puigdomènech $P$, et al. (2002) Differential distribution of PDE4D splice variant mRNAs in rat brain suggests association with specific pathways and presynaptical localization. Synapse 45:259-69. doi: 10.1002/syn.10100

Molinuevo JL, Lladó A, Rami L Memantine: targeting glutamate excitotoxicity in Alzheimer's disease and other dementias. Am J Alzheimers Dis Other Demen 20:77-85.

Montminy MR BL (1987) Binding of a nuclear protein to the cAMP response element of the somatostatin gene. Nature 328:175-179.

Mori F, Pérez-Torres S, De Caro R, et al. (2010) The human area postrema and other nuclei related to the emetic reflex express cAMP phosphodiesterases $4 B$ and $4 D$. J Chem Neuroanat 40:36-42. 
Mors-Fletchtner M, Jenkinson CP, Alt A, et al. (2005) STUDIES OF PHOSPHODIESTERASE EFFECTS ON ADIPOSE tissue metabolism in obese subjects by the microdialysis technique. Physiol Pharmacol 56:355-368.

Moyers SB (2005) Medications as adjunct therapy for weight loss: approved and off-label agents in use. J Am Diet Assoc 105:948-59. doi: 10.1016/j.jada.2005.03.010

Mulchahey JJ, Regmi a, Sheriff S, et al. (1999) Coordinate and divergent regulation of corticotropin-releasing factor (CRF) and CRF-binding protein expression in an immortalized amygdalar neuronal cell line. Endocrinology 140:251-9.

Mungas D (1991) In-office mental status testing: a practical guide. Geriatrics 46:54-8, 63, 66.

Munjack DJ, Palmer R (1988) Thyroid hormones in panic disorder, panic disorder with agoraphobia, and generalized anxiety disorder. J Clin Psychiatry 49:229-31.

Muschamp JW, Van't Veer a., Parsegian a., et al. (2011) Activation of CREB in the Nucleus Accumbens Shell Produces Anhedonia and Resistance to Extinction of Fear in Rats. J Neurosci 31:3095-3103. doi: 10.1523/JNEUROSCI.5973-10.2011

Nakamura J, Okamura N, Kawakami Y (2004) Augmentation of lipolysis in adipocytes from fed rats, but not from starved rats, by inhibition of rolipram-sensitive phosphodiesterase 4 . Arch Biochem Biophys 425:106-14. doi: 10.1016/j.abb.2004.02.036

Nguyen ML, Pirzada MH, Shapiro MA (2013) Zonisamide for weight loss in adolescents. J Pediatr Pharmacol Ther 18:311-314.

Numata S, Iga J-I, Nakataki M, et al. (2009) Gene expression and association analyses of the phosphodiesterase 4B (PDE4B) gene in major depressive disorder in the Japanese population. Am J Med Genet B Neuropsychiatr Genet 150B:527-34. doi: 10.1002/ajmg.b.30852

Numata S, Ueno S-I, Iga J-I, et al. (2008) Positive association of the PDE4B (phosphodiesterase 4B) gene with schizophrenia in the Japanese population. J Psychiatr Res 43:7-12. doi: 10.1016/j.jpsychires.2008.01.013

O'Callaghan JP, Sriram K (2004) Focused microwave irradiation of the brain preserves in vivo protein phosphorylation: comparison with other methods of sacrifice and analysis of multiple phosphoproteins. J Neurosci Methods 135:159-68. doi: 10.1016/j.jneumeth.2003.12.006

O'Connell JC, McCallum JF, McPhee I, et al. (1996) The SH3 domain of Src tyrosyl protein kinase interacts with the N-terminal splice region of the PDE4A cAMP-specific phosphodiesterase RPDE-6 (RNPDE4A5). Biochem J 318 ( Pt 1:255-61. 
O'Donnell JM, Xu Y (2012) Evidence for global reduction in brain cyclic adenosine monophosphate signaling in depression. Biol Psychiatry 72:524-5. doi: 10.1016/j.biopsych.2012.07.017

O'Donnell JM, Zhang H-T (2004) Antidepressant effects of inhibitors of cAMP phosphodiesterase (PDE4). Trends Pharmacol Sci 25:158-63. doi: 10.1016/j.tips.2004.01.003

O'Rahilly S, Farooqi IS (2006) Genetics of obesity. Philos Trans R Soc Lond B Biol Sci 361:1095105. doi: $10.1098 /$ rstb.2006.1850

Ogden C, Carroll M, Kit B, Flegal K (2012) Prevalence of Obesity in the United States , 2009 2010. NCHS Data Brief 1-8.

Olfson M, Marcus SC (2009) National patterns in antidepressant medication treatment. Arch Gen Psychiatry 66:848-56. doi: 10.1001/archgenpsychiatry.2009.81

Olivares EL, Silva-Almeida C, Pestana FM, et al. (2012) Social stress-induced hypothyroidism is attenuated by antidepressant treatment in rats. Neuropharmacology 62:446-56. doi: 10.1016/j.neuropharm.2011.08.035

Olivier B, Soudijn W, van Wijngaarden I (1999) The 5-HT1A receptor and its ligands: structure and function. Prog Drug Res 52:103-65.

Omar B, Banke E, Ekelund M, et al. (2011) Alterations in cyclic nucleotide phosphodiesterase activities in omental and subcutaneous adipose tissues in human obesity. Nutr Diabetes 1:e13. doi: 10.1038/nutd.2011.9

Omori K, Kotera J (2007) Overview of PDEs and their regulation. Circ Res 100:309-27. doi: 10.1161/01.RES.0000256354.95791.f1

Orefice LL, Waterhouse EG, Partridge JG, et al. (2013) Distinct roles for somatically and dendritically synthesized brain-derived neurotrophic factor in morphogenesis of dendritic spines. J Neurosci 33:11618-32. doi: 10.1523/JNEUROSCI.0012-13.2013

Owens RJ, Catterall C, Batty D, et al. (1997) Human phosphodiesterase 4A; characterization of full-length and truncated enzymes expressed in COS cells. Biochem J 326:53-60.

Palmqvist S, Hansson O, Minthon L, Londos E (2009) Practical suggestions on how to differentiate dementia with Lewy bodies from Alzheimer's disease with common cognitive tests. Int J Geriatr Psychiatry 24:1405-12. doi: 10.1002/gps.2277

Park S-J, Ahmad F, Philp A, et al. (2012) Resveratrol ameliorates aging-related metabolic phenotypes by inhibiting CAMP phosphodiesterases. Cell 148:421-33. doi:

10.1016/j.cell.2012.01.017 
Patterson SL, Abel T, Deuel TAS, et al. (1996) Recombinant BDNF rescues deficits in Basal Synaptic Transmission and Hippocampal LTP in BDNF Knockout Mice. Cell 16:1137-1145.

Patterson SL, Pittenger C, Morozov A, et al. (2001) Some forms of cAMP-mediated long-lasting potentiation are associated with release of BDNF and nuclear translocation of phosphoMAP kinase. Neuron 32:123-40.

Perez-Torres S, Miro X, Palacios JM, et al. (2000) Phosphodiesterase type 4 isozymes expression in human brain examined by in situ hybridization histochemistry and [ $3 \mathrm{H}$ ] rolipram binding autoradiography Comparison with monkey and rat brain. J Chem Neuroanat 20:349-374.

Perls TT (2006) The different paths to 100. Am J Clin Nutr 83:484S-487S.

Peters A, Morrison JH, Rosene DL, Hyman BT (1998) Are neurons lost from the primate cerebral cortex during normal aging? Cereb Cortex 8:295-300.

Pies R (2009) Should psychiatrists use atypical antipsychotics to treat nonpsychotic anxiety? Psychiatry (Edgmont) 6:29-37.

Pinner N a, Hamilton L a, Hughes A (2012) Roflumilast: a phosphodiesterase-4 inhibitor for the treatment of severe chronic obstructive pulmonary disease. Clin Ther 34:56-66. doi: 10.1016/j.clinthera.2011.12.008

Podtelezhnikov A a, Tanis KQ, Nebozhyn M, et al. (2011) Molecular insights into the pathogenesis of Alzheimer's disease and its relationship to normal aging. PLoS One 6:e29610. doi: 10.1371/journal.pone.0029610

Pullarkat SR, Mysels DJ, Tan M, Cowen DS (1998) Coupling of Serotonin 5-HT IB Receptors to Activation of Mitogen-Activated Protein Kinase ( ERK-2 ) and p70 S6 Kinase Signaling Systems.

Qi L, Saberi M, Zmuda E, et al. (2009) Adipocyte CREB promotes insulin resistance in obesity. Cell Metab 9:277-86. doi: 10.1016/j.cmet.2009.01.006

Qui Y, Chen C, Malone T, et al. (1991) Characterization of the memory gene dunce of Drosophila melanogaster. J Mol Biol 222:553-65.

Radley JJ, Arias CM, Sawchenko PE (2006a) Regional differentiation of the medial prefrontal cortex in regulating adaptive responses to acute emotional stress. J Neurosci 26:12967-76. doi: 10.1523/JNEUROSCI.4297-06.2006

Radley JJ, Gosselink KL, Sawchenko PE (2009) A discrete GABAergic relay mediates medial prefrontal cortical inhibition of the neuroendocrine stress response. J Neurosci 29:733040. doi: 10.1523/JNEUROSCI.5924-08.2009 
Radley JJ, Rocher AB, Miller M, et al. (2006b) Repeated stress induces dendritic spine loss in the rat medial prefrontal cortex. Cereb Cortex 16:313-20. doi: 10.1093/cercor/bhi104

Radley JJ, Sawchenko PE (2011) A common substrate for prefrontal and hippocampal inhibition of the neuroendocrine stress response. J Neurosci 31:9683-95. doi:

10.1523/JNEUROSCI.6040-10.2011

Radley JJ, Sisti HM, Hao J, et al. (2004) Chronic behavioral stress induces apical dendritic reorganization in pyramidal neurons of the medial prefrontal cortex. Neuroscience 125:16. doi: 10.1016/j.neuroscience.2004.01.006

Radley JJ, Williams B, Sawchenko PE (2008) Noradrenergic innervation of the dorsal medial prefrontal cortex modulates hypothalamo-pituitary-adrenal responses to acute emotional stress. J Neurosci 28:5806-16. doi: 10.1523/JNEUROSCI.0552-08.2008

Raina P, Santaguida P, Ismaila A, et al. (2008) Effectiveness of Cholinesterase Inhibitors and Memantine for Treating Dementia : Evidence Review for a Clinical Practice Guideline. Ann. Intern. Med. 148:

Ramos A (2008) Animal models of anxiety: do I need multiple tests? Trends Pharmacol Sci 29:493-8. doi: 10.1016/j.tips.2008.07.005

Ramos BP, Stark D, Verduzco L, et al. (2006) a2A-adrenoceptor stimulation improves prefrontal cortical regulation of behavior through inhibition of CAMP signaling in aging animals. Learn Mem 13:770-776. doi: 10.1101/Im.298006.Thus

Randt CT, Judge ME, Bonnet KA, Quartermain D (1982) Brain cyclic AMP and memory in mice. Pharmacol Biochem Behav 17:677-80.

Reeves ML, Leigh BK, England PJ (1987) The identification of a new cyclic nucleotide phosphodiesterase activity in human and guinea-pig cardiac ventricle. Implications for the mechanism of action of selective phosphodiesterase inhibitors. Biochem J 241:535-41.

Rena G, Begg F, Ross a, et al. (2001) Molecular cloning, genomic positioning, promoter identification, and characterization of the novel cyclic amp-specific phosphodiesterase PDE4A10. Mol Pharmacol 59:996-1011.

Reusch JEB, Colton LA, Klemm DJ (2000) CREB Activation Induces Adipogenesis in CREB Activation Induces Adipogenesis in 3T3-L1 Cells. doi: 10.1128/MCB.20.3.10081020.2000.Updated

Richter JA, Perry EK, Tomlinson BE (1980) Acetylcholine and choline levels in post-mortem human brain tissue: Preliminary observations in Alzheimer's disease. Life Sci 26:16831689. doi: 10.1016/0024-3205(80)90176-9 
Richter W, Jin S-LC, Conti M (2005) Splice variants of the cyclic nucleotide phosphodiesterase PDE4D are differentially expressed and regulated in rat tissue. Biochem J 388:803-11. doi: 10.1042/BJ20050030

Richter W, Menniti F, Zhang H, Conti M (2013) PDE4 as a target for cognition enhancement. Expert Opin Ther Targets 17:1011-27.

Richter W, Unciuleac L, Hermsdorf T, et al. (2001) Identification of substrate specificity determinants in human CAMP-specific phosphodiesterase $4 \mathrm{~A}$ by single-point mutagenesis. Cell Signal 13:159-67.

Robichaud A, Savoie C, Stamatiou PB, et al. (2001) PDE4 inhibitors induce emesis in ferrets via a noradrenergic pathway. Neuropharmacology 40:262-9.

Robichaud A, Savoie C, Stamatiou PB, et al. (2002a) Assessing the emetic potential of PDE4 inhibitors in rats. Br J Pharmacol 135:113-8. doi: 10.1038/sj.bjp.0704457

Robichaud A, Stamatiou PB, Jin S-LC, et al. (2002b) Deletion of phosphodiesterase 4D in mice shortens alpha(2)-adrenoceptor-mediated anesthesia, a behavioral correlate of emesis. J Clin Invest 110:1045-52. doi: 10.1172/JCl15506

Roman G (1999) A historical review of the concept of vascular dementia: lessons from the past for the future. Alzheimer Dis Assoc Disord 13:s4-8.

Roozendaal B, McEwen BS, Chattarji S (2009) Stress, memory and the amygdala. Nat Rev Neurosci 10:423-33. doi: 10.1038/nrn2651

Rowe WB, Spreekmeester E, Meaney MJ, et al. (1998) Reactivity to novelty in cognitivelyimpaired and cognitively-unimpaired aged rats and young rats. Neuroscience 83:669-80.

Russo SJ, Murrough JW, Han M-H, et al. (2012) Neurobiology of resilience. Nat Neurosci 15:1475-1484. doi: 10.1038/nn.3234

Rutten K, Misner DL, Works M, et al. (2008) Enhanced long-term potentiation and impaired learning in phosphodiesterase 4D-knockout (PDE4D) mice. Eur J Neurosci 28:625-32. doi: 10.1111/j.1460-9568.2008.06349.x

Sakkas P, Mihalopoulou P, Mourtzouhou P, et al. (2003) Induction of mania by rTMS: report of two cases. Eur Psychiatry 18:196-8.

Saldou N, Obernolte R, Huber a, et al. (1998) Comparison of recombinant human PDE4 isoforms: interaction with substrate and inhibitors. Cell Signal 10:427-40.

Salmon P (2001) Effects of physical exercise on anxiety, depression, and sensitivity to stress: a unifying theory. Clin Psychol Rev 21:33-61. 
Scheltens P, Barkhof F, Leys D, Wolters EC (1995) Histopathologic correlates of white-matter changes on MRI in Alzheimer's disease and normal aging. Neurology 45:883-888.

Schwartz E, Mobbs C V (2012) Hypothalamic BDNF and obesity: found in translation. Nat Med 18:496-497. doi: 10.1038/nm.2716

Scott Al, Perini AF, Shering PA, Whalley LJ (1991) In-patient major depression: is rolipram as effective as amitriptyline? Eur J Clin Pharmacol 40:127-9.

Sebastiani G, Morissette C, Lagacé C, et al. (2006) The cAMP-specific phosphodiesterase 4B mediates Abeta-induced microglial activation. Neurobiol Aging 27:691-701. doi: 10.1016/j.neurobiolaging.2005.03.024

Sekiyama K, Hashimoto O, Ushiro Y, et al. (2009) Abnormalities in aggression and anxiety in transgenic mice overexpressing activin E. Biochem Biophys Res Commun 385:319-23. doi: 10.1016/j.bbrc.2009.05.054

Services H (2010) Centers for disease control and prevention, Morbidity and Mortality Weekly Report. 59:

Shackman AJ, Sarinopoulos I, Maxwell JS, et al. (2006) Anxiety selectively disrupts visuospatial working memory. Emotion 6:40-61. doi: 10.1037/1528-3542.6.1.40

Shah A a, Sjovold T, Treit D (2004) Inactivation of the medial prefrontal cortex with the GABAA receptor agonist muscimol increases open-arm activity in the elevated plus-maze and attenuates shock-probe burying in rats. Brain Res 1028:112-5. doi: 10.1016/j.brainres.2004.08.061

Shah A a, Treit D (2003) Excitotoxic lesions of the medial prefrontal cortex attenuate fear responses in the elevated-plus maze, social interaction and shock probe burying tests. Brain Res 969:183-94.

Shah A a, Treit D (2004) Infusions of midazolam into the medial prefrontal cortex produce anxiolytic effects in the elevated plus-maze and shock-probe burying tests. Brain Res 996:31-40. doi: 10.1016/j.brainres.2003.10.015

Shao M, Yi X, Chi L, et al. (2014) Ischemic stroke risk in a southeastern Chinese population: Insights from 5-lipoxygenase activating protein and phosphodiesterase 4D singlenucleotide polymorphisms. J Formos Med Assoc. doi: 10.1016/j.jfma.2013.12.004

Sharma S, Fulton S (2012) Diet-induced obesity promotes depressive-like behavior that is associated with neural adaptations in brain reward circuitry. Int J Obes.

Shaywitz AJ, Greenberg ME (1999) CREB: a stimulus-induced transcription factor activated by a diverse array of extracellular signals. Annu Rev Biochem 68:821-861. 
Shefer V (1973) Shefer VF (1973) Absolute number of neurons and thickness of cerebral cortex during aging, senile and vascular dementia, and Pick's and Alzheimer's diseases. Neurosci Behav Physiol 6:319-24.

Shelton RC, Osuntokun O, Heinloth AN, Corya S a (2010) Therapeutic options for treatmentresistant depression. CNS Drugs 24:131-61. doi: 10.2165/11530280-000000000-00000

Shepherd M, McSorley T, Olsen AE, et al. (2003) Molecular cloning and subcellular distribution of the novel PDE4B4 CAMP-specific phosphodiesterase isoform. Biochem J 370:429-38. doi: 10.1042/BJ20021082

Sheriff S, Dautzenberg FM, Mulchahey JJ, et al. (2001) Interaction of neuropeptide $Y$ and corticotropin-releasing factor signaling pathways in AR-5 amygdalar cells. Peptides 22:2083-9.

Shieh PB, Ghosh A (1999) Molecular mechanisms underlying activity-dependent regulation of BDNF expression. Dev Neurobiol 41:127-34.

Sierksma a SR, van den Hove DL a, Pfau F, et al. (2013) Improvement of spatial memory function in APPswe/PS1dE9 mice after chronic inhibition of phosphodiesterase type 4D. Neuropharmacology 1-12. doi: 10.1016/j.neuropharm.2013.09.015

Sierra-Mercado D, Corcoran K a, Lebrón-Milad K, Quirk GJ (2006) Inactivation of the ventromedial prefrontal cortex reduces expression of conditioned fear and impairs subsequent recall of extinction. Eur J Neurosci 24:1751-8. doi: 10.1111/j.14609568.2006.05014.x

Silvestre J, Fernandez A, Palacios J (1999) Effects of rolipram on the elevated plus-maze test in rats: a preliminary study. J Psychophrmacology 13:274-7.

Simon GE, Korff M Von, Saunders K, Diana L (2007) ASSOCIATION BETWEEN OBESITY AND PSYCHIATRIC DISORDERS IN THE US ADULT POPULATION. Arch Gen Psychiatry 63:824-830.

Skullerud K (1985) Variations in the size of the human brain. Influence of age, sex, body length, body mass index, alcoholism, Alzheimer changes, and cerebral atherosclerosis. Acta Neurol Scand 102:1-94.

Smith WB, McCaslin IR, Gokce a, et al. (2013) PDE5 inhibitors: considerations for preference and long-term adherence. Int J Clin Pract 67:768-80. doi: 10.1111/ijcp.12074

Snyder PB, Esselstyn JM, Loughney K, et al. (2005) The role of cyclic nucleotide phosphodiesterases in the regulation of adipocyte lipolysis. J Lipid Res 46:494-503. doi: 10.1194/jlr.M400362-JLR200 
Sramek JJ, Zarotsky V, Cutler NR (2002) Generalised Anxiety Disorder Treatment Options. Drugs 62:1635-1648.

Stahl SM, Ph D (2002) Don't ask, Don't Tell, but Benzodiazepines Are Still the Leading Treatments for Anxiety Disorder. J Clin Psychiatry 63:

Stair SL, Rainnie D (2011) A comparison of Phosphodiesterase Type 4 (PDE4) Expression in the BLA in Control and Chronically Stressed Rats. Emory Honor. Thesis

Steimer T (2011) Animal models of anxiety disorders in rats and mice: come conceptual issues. Dialogues Clin Neurosci 13:495-506.

Stein DJ, Ahokas A, Márquez MS, et al. (2014) Agomelatine in generalized anxiety disorder: an active comparator and placebo-controlled study. J Clin Psychiatry. doi: 10.4088/JCP.13m08433

Stein DJ, Ahokas AA, de Bodinat C (2008) Efficacy of agomelatine in generalized anxiety disorder: a randomized, double-blind, placebo-controlled study. J Clin Psychopharmacol 28:561-6. doi: 10.1097/JCP.0b013e318184ff5b

Stein DJ, Picarel-Blanchot F, Kennedy SH (2013) Efficacy of the novel antidepressant agomelatine for anxiety symptoms in major depression. Hum Psychopharmacol 28:151-9. doi: 10.1002/hup.2294

Streitberger K, Steppan J, Maier C, et al. (2008) Effects of verum acupuncture compared to placebo acupuncture on quantitative EEG and heart rate variability in healthy volunteers. J Altern Complement Med 14:505-13. doi: 10.1089/acm.2007.0552

Ströhle A (2009) Physical activity, exercise, depression and anxiety disorders. J Neural Transm 116:777-84. doi: 10.1007/s00702-008-0092-x

Sullivan M, Rena G, Begg F, et al. (1998) Identification and characterization of the human homologue of the short PDE4A cAMP-specific phosphodiesterase RD1 (PDE4A1) by analysis of the human HSPDE4A gene locus located at chromosome 19p13.2. Biochem J 333:693703.

Summary E (2011) Off-Label Use of Atypical Antipsychotics : An Update. Comp. Eff. Rev.

Swinnen J V, Joseph DR, Conti M (1989) Molecular cloning of rat homologues of the Drosophila melanogaster dunce cAMP phosphodiesterase: evidence for a family of genes. Proc Natl Acad Sci U S A 86:5325-9.

Tao X, Finkbeiner S, Arnold DB, et al. (1998) Ca2+ influx regulates BDNF transcription by a CREB family transcription factor-dependent mechanism. Neuron 20:709-726. 
Tavares RF, Corrêa FM a, Resstel LBM (2009) Opposite role of infralimbic and prelimbic cortex in the tachycardiac response evoked by acute restraint stress in rats. J Neurosci Res 87:2601-7. doi: 10.1002/jnr.22070

Terry R, DeTeresa R, Hansen L (1987) Neocortical cell counts in normal human adult aging. Ann Neurol 21:530-9.

Varada S, Tintle SJ, Gottlieb AB (2014) Apremilast for the treatment of psoriatic arthritis. Expert Rev Clin Pharmacol 7:239-50. doi: 10.1586/17512433.2014.904200

Vasconcellos a. PS, Tabajara a. S, Ferrari C, et al. (2003) Effect of chronic stress on spatial memory in rats is attenuated by lithium treatment. Physiol Behav 79:143-149. doi: 10.1016/S0031-9384(03)00113-6

Vecsey CG, Baillie GS, Jaganath D, et al. (2009) Sleep deprivation impairs CAMP signaling in the hippocampus. Nature 461:1122-1125. doi: 10.1038/nature08488.Sleep

Venero C, Guadaño-Ferraz A, Herrero Al, et al. (2005) Anxiety, memory impairment, and locomotor dysfunction caused by a mutant thyroid hormone receptor alpha1 can be ameliorated by T3 treatment. Genes Dev 19:2152-63. doi: 10.1101/gad.346105

Verde I, Pahlke G, Salanova M, et al. (2001) Myomegalin is a novel protein of the golgi/centrosome that interacts with a cyclic nucleotide phosphodiesterase. J Biol Chem 276:11189-98. doi: 10.1074/jbc.M006546200

Vertes RP (2004) Differential projections of the infralimbic and prelimbic cortex in the rat. Synapse 51:32-58. doi: 10.1002/syn.10279

Vigers a J, Amin DS, Talley-Farnham T, et al. (2012) Sustained expression of brain-derived neurotrophic factor is required for maintenance of dendritic spines and normal behavior. Neuroscience 212:1-18. doi: 10.1016/j.neuroscience.2012.03.031

Vyas A, Mitra R, Shankaranarayana Rao BS, Chattarji S (2002) Chronic stress induces contrasting patterns of dendritic remodeling in hippocampal and amygdaloid neurons. J Neurosci 22:6810-8. doi: 20026655

Wachtel H (1983) Potential antidepressant activity of rolipram and other selective cyclic adenosine $3^{\prime}, 5^{\prime}$-monophosphate phosphodiesterase inhibitors. Neuropharmacology 22:267-72.

Wachtel H, Schneider H (1986) Rolipram, a novel antidepressant drug, reverses the hypothermia and hypokinesia of monoamine-depleted mice by an action beyond postsynaptic monoamine receptors. Neuropharmacology 25:1119-26. 
Waddleton D, Wu W, Feng Y, et al. (2008) Phosphodiesterase 3 and 4 comprise the major cAMP metabolizing enzymes responsible for insulin secretion in INS-1 (832/13) cells and rat islets. Biochem Pharmacol 76:884-93. doi: 10.1016/j.bcp.2008.07.025

Wadzinski BE, Wheat WH, Jaspers S, et al. (1993) Nuclear Protein Phosphatase 2A Dephosphorylates Protein Kinase A-Phosphorylated CREB and Regulates CREB Transcriptional Stimulation. Mol Cell Biol 13:2822-2834. doi: 10.1128/MCB.13.5.2822.Updated

Wallace DA, Johnston LA, Huston E, et al. (2005) Identification and Characterization of PDE4A11 , a Novel , Widely Expressed Long Isoform Encoded by the Human PDE4A cAMP Phosphodiesterase Gene. Mol Pharmacol 67:1920-1934. doi: 10.1124/mol.104.009423. The

Wallace TL, Stellitano KE, Neve RL, Duman RS (2004) Effects of cyclic adenosine monophosphate response element binding protein overexpression in the basolateral amygdala on behavioral models of depression and anxiety. Biol Psychiatry 56:151-60. doi: 10.1016/j.biopsych.2004.04.010

Wang H, Edens NK (2007) mRNA expression and antilipolytic role of phosphodiesterase 4 in rat adipocytes in vitro. J Lipid Res 48:1099-107. doi: 10.1194/jlr.M600519-JLR200

Wang H, Peng M-S, Chen Y, et al. (2007a) Structures of the four subfamilies of phosphodiesterase-4 provide insight into the selectivity of their inhibitors. Biochem J 408:193-201. doi: 10.1042/BJ20070970

Wang H, Qi H, Wang B-S, et al. (2008) Is acupuncture beneficial in depression: a meta-analysis of 8 randomized controlled trials? J Affect Disord 111:125-34. doi: 10.1016/j.jad.2008.04.020

Wang M, Ramos BP, Paspalas CD, et al. (2007b) Alpha2A-adrenoceptors strengthen working memory networks by inhibiting CAMP-HCN channel signaling in prefrontal cortex. Cell 129:397-410. doi: 10.1016/j.cell.2007.03.015

Wang P, Myers JG, Wu P, et al. (1997) Expression, Purification, and Characterization of Human Subtypes A , B , C , and D. Biochem Biophys Res Commun 234:320-324.

Wang Z, Zhang Y, Liu Y, et al. (2013) RNA interference-mediated phosphodiesterase 4D splice variants knock-down in the prefrontal cortex produces antidepressant-like and cognitionenhancing effects. Title. Br J Pharmacol 168:1004-14.

Williamson RA, Davison TF (1987) Effect of increased circulating corticosterone on serum and thyroidal concentrations of iodothyronines and the responses to thyrotrophin in the immature fowl (Gallus domesticus). Gen Comp Endocrinol 65:65-72. 
Winters BD, Saksida LM, Bussey TJ (2008) Object recognition memory: neurobiological mechanisms of encoding, consolidation and retrieval. Neurosci Biobehav Rev 32:1055-70. doi: 10.1016/j.neubiorev.2008.04.004

Xu B, Gottschalk W, Chow A, et al. (2000) The Role of Brain-Derived Neurotrophic Factor Receptors in the Mature Hippocampus: Modulation of Long-Term Potentiation through a Presynaptic Mechanism involving TrkB. J Neurosci 20:6888-6897.

Yan Y, Luo X, Zhang J, et al. (2014) Association between phosphodiesterase 4D polymorphism SNP83 and ischemic stroke. J Neurol Sci 338:3-11. doi: 10.1016/j.jns.2013.12.012

Ye Y, Jackson K, O'Donnell JM (2000) Effects of repeated antidepressant treatment of type 4A phosphodiesterase (PDE4A) in rat brain. J Neurochem 74:1257-62.

Yen Y-C, Mauch CP, Dahlhoff M, et al. (2012) Increased levels of conditioned fear and avoidance behavior coincide with changes in phosphorylation of the protein kinase $B(A K T)$ within the amygdala in a mouse model of extremes in trait anxiety. Neurobiol Learn Mem 98:56-65. doi: 10.1016/j.nIm.2012.04.009

Zeller E, Stief HJ, Pflug B, Sastre-y-Hernández M (1984) Results of a phase II study of the antidepressant effect of rolipram. Pharmacopsychiatry 17:188-90. doi: 10.1055/s-20071017435

Zhang H-T (2009) Cyclic AMP-specific phosphodiesterase-4 as a target for the development of antidepressant drugs. Curr Pharm Des 15:1688-98.

Zhang H-T, Huang Y, Jin S-L, et al. (2002) Antidepressant-like profile and reduced sensitivity to rolipram in mice deficient in the PDE4D phosphodiesterase enzyme.

Neuropsychopharmacol Off Publ Am Coll Neuropsychopharmacol 27:587-95. doi: 10.1016/S0893-133X(02)00344-5

Zhang H-T, Huang Y, Masood A, et al. (2008) Anxiogenic-like behavioral phenotype of mice deficient in phosphodiesterase 4B (PDE4B). Neuropsychopharmacology 33:1611-23. doi: 10.1038/sj.npp.1301537

Zhang H-T, Zhao Y, Huang Y, et al. (2006) Antidepressant-like effects of PDE4 inhibitors mediated by the high-affinity rolipram binding state (HARBS) of the phosphodiesterase-4 enzyme (PDE4) in rats. Psychopharmacology (Berl) 186:209-17. doi: 10.1007/s00213-0060369-4

Zhang R, Maratos-Flier E, Flier JS (2009) Reduced adiposity and high-fat diet-induced adipose inflammation in mice deficient for phosphodiesterase 4B. Endocrinology 150:3076-82. doi: 10.1210/en.2009-0108 
Zhao AZ (2005) Control of Food Intake Through Regulation of cAMP. Curr Top Dev Biol 67:207224. doi: 10.1016/S0070-2153(04)67006-2 


\author{
Rolf T. Hansen III \\ 1 Medical Center Drive \\ HSC North, Department of Neurobiology and Anatomy \\ Room 4028 \\ Morgantown, WV 26506 \\ Phone: (304) 293-4782 \\ Email: rhansen1@mix.wvu.edu
}

\title{
Education
}

\begin{tabular}{|c|c|}
\hline $09 / 14$ & $\begin{array}{l}\text { University of Pennsylvania } \\
\text { Post-doctoral fellow } \\
\text { Laboratory of Dr. Ted Abel }\end{array}$ \\
\hline $08 / 08-06 / 14$ & $\begin{array}{l}\text { West Virginia University } \\
\text { Ph.D. Candidate in neuroscience } \\
\text { Advisor: Dr. Han-Ting Zhang }\end{array}$ \\
\hline 08/02-05/06 & $\begin{array}{l}\text { Gannon University } \\
\text { B.S. In Biology, graduated cum laud }\end{array}$ \\
\hline
\end{tabular}

\section{Research Experience}

06/09-Present

09/06-06/08

01/06-06/06
Graduate Student, West Virginia University, Department Of Neurobiology and Anatomy

PDE4 subtypes in memory, anxiety, and depression. Currently investigating the role the individual PDE4 subtypes A, B, and D play in memory, anxiety, and depression. Techniques involve extensive behavioral testing, knockout mice, western blotting, ELISA, cell culture, and immunohistochemistry.

Novel sigma ligands and methamphetamine abuse. Investigated the role that the novel sigma ligand AZ66 played in methamphetamine abuse and cognition.

EPAC and Alzheimer's disease. Investigated the relationship between EPAC and Alzheimer's disease. Preliminary research included the use of stereotactic surgery, cortical cell cultures, multiple behavior tests, western blotting, PCR, ELISA, and immunohistochemistry.

Chemical Engineer, PPG Industries, Optical Polymer Division

Research on High Index Lens Polymer-Researched and developed a proprietary high index, high durability lens polymer for consumer eye wear. Responsibilities included the supervision and maintenance of the R\&D polymer casting machine, designing, running and recording of experiments, inventory and maintenance of the lab, and also customer demonstrations and presentations.

Undergraduate Student, Gannon University 
Research on E. faecalis vancomycin resistance-Researched the inheritance of novel vancomycin resistance genes by $E$. faecalis in the waters of Lake Erie. This research was performed by using multiple microbiological tests, PCR, DNA sequencing, cloning, and gel electrophoresis.

\section{Peer Reviewed Publications}

$2 / 14$

$2 / 14$

$4 / 13$

$8 / 12$

$4 / 11$
Hansen R.T., Zhang H.-T. Phosphodiesterase-4 modulation as a future therapeutic for cognitive loss in pathological- and non-pathological -aging: Possibilities and Pitfalls. Current Pharmaceutical Design (In Press).

Hansen R.T., Conti M, Zhang H.-T. Mice deficient in phosphodiesterase-4A display anxiogenic-like behavior. Psychopharmacology 2014 [epub ahead of print].

Hansen R. T., Zhang H.-T. Senescent-induced dysregulation of CAMP/CREB signaling and correlations with cognitive decline. Brain research, 2013 Jun. 1516, 93-109.

Seminerio M, Hansen R, Kaushal N, Zhang HT, McCurdy C, Matsumoto R. The Evaluation of AZ66, an Optimized Sigma Receptor Ligand, Against Methamphetamine-Induced Dopaminergic Neurotoxicity and Cognitive Impairment. Int J Neuropsychopharmacol. 2012 Aug 29:1-12.

Hu W, Lu T, Chen A, Huang Y, Hansen R, Chandler LJ, Zhang HT. Inhibition of phosphodiesterase-4 decreases ethanol intake in mice. Psychopharmacology, 2011, 218:331-339.

\section{Honors/Awards}

4/14 Appointed to postdoctoral T32 fellowship at the University of Pennsylvania to study the molecular mechanisms of schizophrenia

5/13 Winner of the 2013 WVU Dissertation Fellowship.

Awarded a $\$ 6,000$ fellowship to fund dissertation work for the fall semester of 2013

$09 / 12$

Winner of the 2012 Stereology Fellowship from the Stereology Resource Center

$03 / 12$

Received second Place at WVU Van Liere research day for Oral Presentation "Phosphodiesterase 4A as a Potential Target for Anxiety and Obesity."

$07 / 11$

Awarded a $\$ 1,700$ scholarship to attend the WVU Science and Technology Entrepreneurship Academy

Received first place at WVU Van Liere research day for best poster- "Behavioral phenotype of phosphodiesterase 4A knockout mice suggests a role in memory and anxiety" in 08' Basic Science Category 
$09 / 09$

09/06-06/08

$03 / 06$
Appointed to NIH pre-doctoral T32 training grant in the behavioral and biomedical sciences

Received two level 1 achievement awards at PPG to recognize outstanding contribution to team, research, and dedicated performance.

Nominated for the Schubert Award, Gannon University's award for academic and social excellence in biology.

\section{Teaching Experience}

05/10-Present Assisted with the teaching and training of multiple undergraduate students, graduate students, medical students, post docs, and visiting scholars

10/05-04/06 Immunology Lab Assistant-Gannon University Taught undergraduate students introductory immunological concepts alongside professor. Other responsibilities included preparing chemicals/materials needed for lab, assisting students, grading papers, and supervising the lab to ensure suitable learning environment.

\section{Memberships}

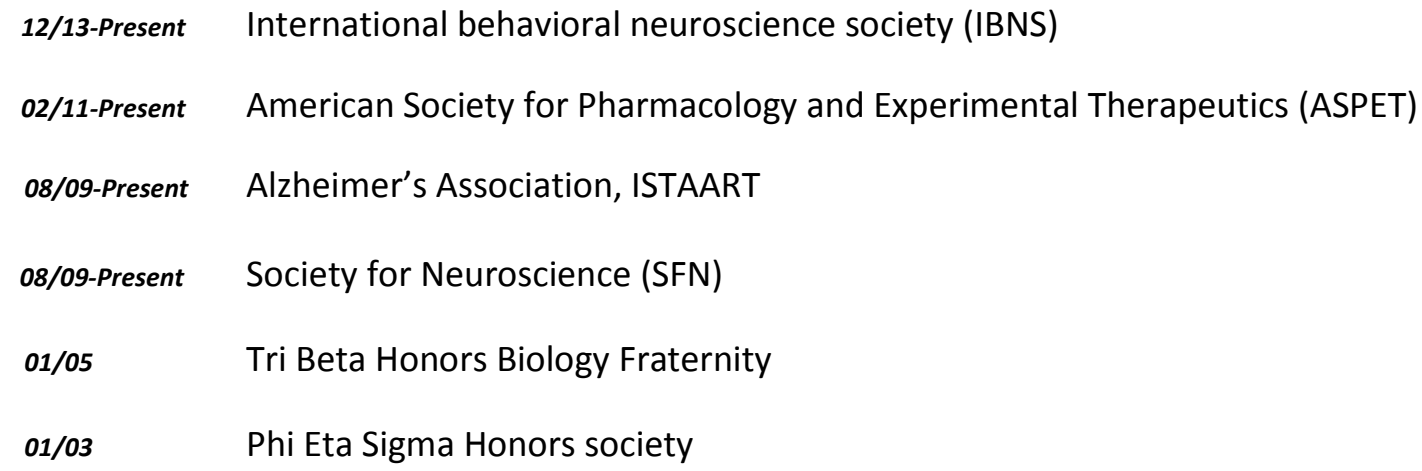

\section{Service/Committees}

6/13-Present

03/13-Present
Appointed to the steering committee for the "WVU Neuroscience Seminar Series" at the WVU Center for Neuroscience.

Nominated and chosen to serve on the WVU School of Medicine Biomedical Program Admissions Committee Responsibilities included selecting, interviewing, and choosing potential candidates for the WVU Biomedical Program. 


\begin{abstract}
1/13-Present Appointed as Social Chair of the Graduate Student Organization (GSO)
Head of the social committee of which the responsibilities included organizing, funding, and promoting activities beneficial to graduate life at WVU

08/12-Present Appointed to the WVU Graduate Student Organization-Neuroscience Program Representative

Responsibilities included representing the interests of the WVU Graduate Student body to faculty and administration; organizing, promoting and conducting activities beneficial to graduate life at WVU; and organizing, promoting, and conducting activities beneficial to the greater Morgantown area community.

06/11-Present Served on the WVU Van Liere Research Day Committee

Responsibilities included assisting with the organization of the WVU Van Liere research day.

09/09-Present Served on the Behavioral and Biomedical Sciences (BBS) T32 Training Scholarship Funding Committee. Responsibilities included the appropriation of $\mathbf{\$ 2 0 , 0 0 0}$ of $\mathbf{N I H ~ g r a n t}$ money to applicants wishing to fund their research.
\end{abstract}

\title{
Peer-Reviewing Experience
}

12/13 Guest language editor for "Current Pharmaceutical Design"

7/25/13 Invited to serve as a reviewer for "Behavioral and Brain functions"

2/12 Invited to serve as a reviewer for the "Evidence-Based Complementary and Alternative Medicine"

12/11-Present Invited to serve as a reviewer for "Metabolic Brain Disease"

\section{Invited Talks}

$3 / 14$

$10 / 12$

$3 / 12$

$06 / 10$
"Functional characterization of Phosphodiesterase-4A in Behavior" at the University of Pennsylvania

"Phosphodiesterase-4A as a potential mediator of anxiety like behavior in mice" at the 18th Annual International Stereology Workshop. New Orleans, LA, USA.

"Phosphodiesterase 4A as a Potential Target for Anxiety and Obesity" at WVU Van Liere Research Day

"Molecular basis for changes in behavioral state in ant social behaviors" at the meeting for behavioral and biomedical researchers 


\section{Meeting Presentations/Abstracts}

$10 / 12$

$10 / 12$

07/12

$06 / 12$

05/12

$03 / 12$

$03 / 12$

$02 / 12$

11/11

$06 / 11$

Hansen R, Wu Q, Wang C, Conti M, Zhang HT. Phosphodiesterase-4A Knockout Protects Against Abeta42-Induced Memory Decline. The Society for Neuroscience Annual Meeting. New Orleans, LA.

Matsumoto R, Seminerio M, Hansen R, Kaushal N, McCurdy C, Zhang HT. The evaluation of AZ66, an optimized sigma receptor ligand, against methamphetamine-induced dopaminergic neurotoxicity and memory impairment in mice. The Society for Neuroscience Annual Meeting. New Orleans, LA.

Wang C, Wu Q, Cheng YF, Mannan S, Hansen R, Huang Y, Conti M, Wilson SP, O'Donnell $J M$, Zhang HT. Disruption of phosphodiesterase (PDE) for treating memory loss in Alzheimer's disease. Cognitive Enhancement in Health \& Disease. Federation of European Neuroscience Societies (FENS). Barcelona, Spain.

Jiang YM, Webster P, Hu W, Duke R, Shi D, Doppalapudi S, Huang Y, Hansen R, Zhang C, Meng ZZ, Conti M, Grahame N, Zhang HT. Cyclic nucleotide phosphodiesterases (PDEs): Novel targets for alcohol drinking behavior. Research Society on Alcoholism (RSA) 35th Annual Scientific Meeting, San Francisco, CA, USA.

Jiang YM, Hu W, Webster P, Huang Y, Doppalapudi S, Duke R, Hansen R, Meng ZZ, Conti $\mathrm{M}$, Zhang HT. Role of phosphodiesterase-4 (PDE4) subtypes in the regulation of ethanol consumption. Gordon Research Conference (GRC) on Cyclic Nucleotide PDEs. II Ciocco, Barga, Italy.

Jiang YM, Hu W, Webster P, Hansen R, Huang Y, Doppalapudi S, Meng ZZ, Conti M, Zhang HT. Targeting phosphodiesterase-4 (PDE4) and its subtypes to regulate ethanol consumption. 2012 Appalachian Health Summit/7th Annual CCTS Spring Conference. Lexington, KY, USA.

Hansen R, Conti M, Zhang HT. Phosphodiesterase 4A as a Potential Target for Anxiety and Obesity. WVU Van Liere Research Day. Morgantown, WV, USA.

Hansen R, Conti M, Zhang HT. Phosphodiesterase 4A as a Potential Target for Anxiety and Obesity. WVU LINC Dinner. Morgantown, WV, USA.

Zhang HT, Wang C, Huang Y, Hansen R. Inhibition of phosphodiesterase-4 reverses Abeta1-42-induced memory deficits and neuroinflammation in mice. 41st Society for Neuroscience (SFN) Annual Meeting. Washington, DC, USA.

Hu W, Wen RT, Huang Y, Hansen R, Wang C, Conti M, Grahame N, Liang JH, Zhang HT. Cyclic AMP-specific phosphodiesterase-4 (PDE4): a novel target for alcohol dependence. Research Society on Alcoholism (RSA) 34th Annual Scientific Meeting, Atlanta, GA, USA. 
Hansen R, Wu Q, Wang C, Huang Y, Hu W, Jiang YM, Meng ZZ, Conti M, O'Donnell JM, Zhang HT. Behavioral phenotype of PDE4A knockout mice: Role of PDE4A in memory and anxiety. The Experimental Biology Annual Meeting, American Society for Pharmacology and Experimental Therapeutics (ASPET), Washington, DC, USA.

$03 / 11$

Hansen R, Wu Q, Wang C, Huang Y, Hu W, Jiang YM, Meng ZZ, Conti M, O'Donnell JM, Zhang HT. Behavioral phenotype of PDE4A knockout mice: Role of PDE4A in memory and anxiety. West Virginia University Van Liere Research Day, Morgantown, WV, USA.

Hu W, Wen RT, Huang Y, Hansen R, Wang C, Wu Q, Conti M, Liang JH, Zhang HT. Targeting phosphodiesterase-4 (PDE4) to reduce ethanol intake in rodents. 49th ACNP Annual Meeting, Miami, FL, USA.

$11 / 10$

Wu Q, Hansen R, Pawlak C, Wang C, Cheng YF, Hu W, O'Donnell M, Zhang HT. Beta amyloid 1-42 produced memory impairment and depressive-like behavior via decreased cyclic AMP signaling. 40th Society for Neuroscience (SFN) Annual Meeting, San Diego, CA, USA.

Hu W, Huang Y, Cheng YF, Hansen R, Wang C, Wu Q, Chandler L, Conti M, Zhang HT. Phosphodiesterase-4 (PDE4): A novel target for reducing ethanol intake. 40th Society for Neuroscience (SFN) Annual Meeting, San Diego, CA, USA.

$06 / 10$

Hansen R, O'Donnell J, Zhang HT. Attenuating decreased locomotor activity as a confounding variable in memory tests of Aged Mice. WVU Department of Behavioral Medicine Retreat. Morgantown, WV, USA.

$06 / 10$

Wu Q, Hansen R, Hu W, Huang Y, Wang C, Mannan S, Cheng YF, Conti M, O'Donnell M., Zhang HT. Mice deficient in PDE4A display memory-enhancing, anxiolytic-like, and neurogenic effects via increased cAMP signaling. Gordon Research Conference on Cyclic Nucleotide PDEs, Waterville Valley, NH, USA, 2010

$06 / 10$

$4 / 10$

Hu W, Huang Y, Cheng YF, Hansen R, Wang C, Wu Q, Chandler LJ, Conti M, Zhang HT. Inhibition of Phosphodiesterase-4B Decreases Ethanol Preference in Mice. Gordon Research Conference on Cyclic Nucleotide PDEs, Waterville Valley, NH, USA, 2010

Zhang HT, Wu Q, Hansen R, Mannan S, Cheng YF, Huang Y, Hu W, Conti M, O'Donnell M. Beta amyloid 1-42-induced depressive-like behavior and decreases in adult neurogenesis are mediated by the phosphodiesderase-4D (PDE4D) enzyme. The Experimental Biology Annual Meeting, American Society for Pharmacology and Experimental Therapeutics (ASPET), Anaheim, CA, USA, 2010

$04 / 10$

Hansen R, O'Donnell J, Zhang HT. Attenuating decreased locomotor activity as a confounding variable in memory tests of Aged Mice. WVU Van Liere Research day. . Morgantown, WV, USA.

$03 / 10$

Hansen R, Zhang HT. Epac, a Potential Neuroprotective, Memory Enhancing Target for Alzheimer's Disease. WVU School of Pharmacy Retreat. Morgantown, WV, USA. 


\section{References}

Available upon request 